\title{
Channel Gap Probe EMPIrE Report
}

James A. Smith, Casey J. Jesse, Clark L. Scott and David L. Cottle

September 2018

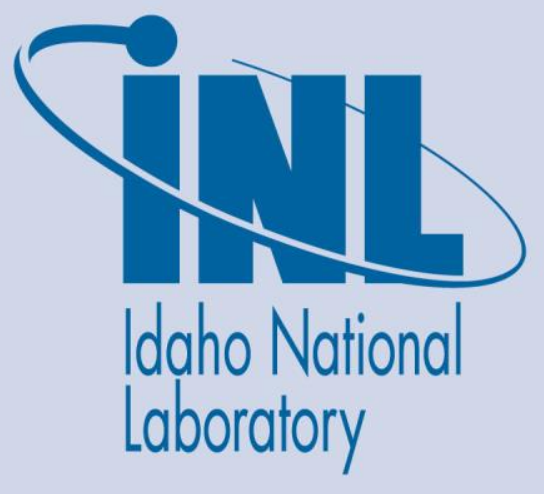

The INL is a U.S. Department of Energy National Laboratory operated by Battelle Energy Alliance 


\section{DISCLAIMER}

This information was prepared as an account of work sponsored by an agency of the U.S. Government. Neither the U.S. Government nor any agency thereof, nor any of their employees, makes any warranty, expressed or implied, or assumes any legal liability or responsibility for the accuracy, completeness, or usefulness, of any information, apparatus, product, or process disclosed, or represents that its use would not infringe privately owned rights. References herein to any specific commercial product, process, or service by trade name, trade mark, manufacturer, or otherwise, does not necessarily constitute or imply its endorsement, recommendation, or favoring by the U.S. Government or any agency thereof. The views and opinions of authors expressed herein do not necessarily state or reflect those of the U.S. Government or any agency thereof. 
INL/EXT-18-51523

Revision 0

\section{Channel Gap Probe EMPIrE Report}

James A. Smith, Casey J. Jesse, Clark L. Scott and David L. Cottle

September 2018

Idaho National Laboratory Idaho Falls, Idaho 83415

http://www.inl.gov

Prepared for the

U.S. Department of Energy

Office of Nuclear Energy

Under DOE Idaho Operations Office

Contract DE-AC07-05ID14517 

Idaho National Laboratory

\title{
Channel Gap Probe EMPIrE Report
}

\author{
INL/EXT-18-51523
}

Revision 0

September 2018

Approved by:
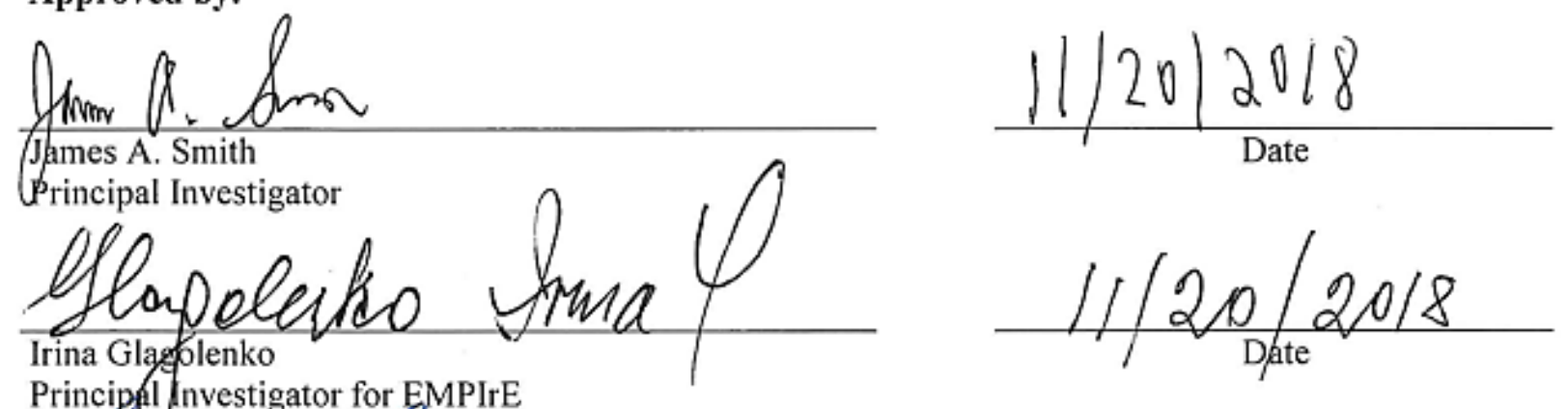

Principel Investigator for EMPIrE
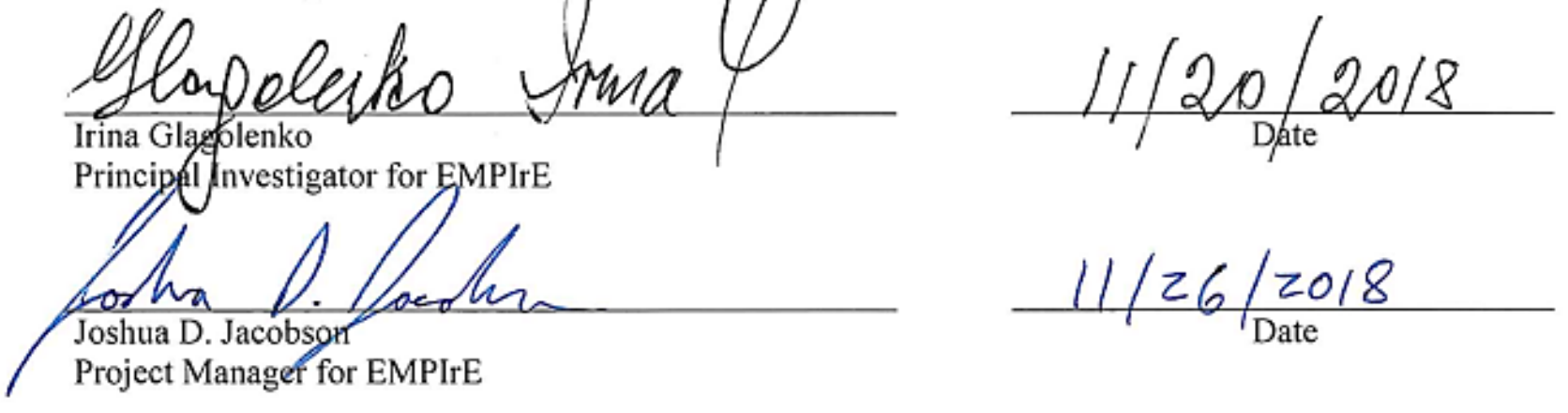


\begin{abstract}
EMPIrE aims to provide data for the "comprehension phase" of the European Low Enriched Uranium (LEU ) U-Mo Fuel Development program for the conversion of European civilian research reactors. The majority of the fuel performance data will be obtained during post-irradiation examinations (PIE) of the fuel. The primary EMPIrE fuel specimen design is based on uraniummolybdenum (U-Mo) alloy particles dispersed in an aluminum matrix clad in the European alloy AG3NE and in the form of mini-plate sized specimens (1" x 4" plates). Secondary EMPIrE specimens include U-Mo monolithic fuel clad in European alloy AlFeNi. The experiment is designed in such a way that U-Mo samples achieve three distinct end-of-life burnup groupings. Irradiation has taken place at ATR (Advance Treatment Reactor).

The fuel plates are contained in fuel capsules holding four plates each, with 2 plates at 2 levels. Once the capsules with the plates are irradiated, they have to be inspected during the outage in-between cycles. The capsules need to have visual inspection performed and channel gap widths measured prior to reinsertion. These inspections are to ensure that the plates have not deformed significantly. Obstruction of the coolant channels and restriction of the coolant flow are less of a concern in EMPIrE testing as the channels were made intentionally wide. The Channel Gap Probe (CGP) has been developed to measure channel gap widths, which serves as indirect measures of the fuel plate performance and deformation in the course of irradiation which enables the evaluation of the risk from continuing irradiation prior to reinsertion of the plates in reactor.
\end{abstract}

\title{
ACKNOWLEDGEMENTS
}

Special thanks to Irina Glagolenko and Margaret Marshall for their efforts and comments, as well as Douglas Crawford and Hakan Ozaltun for their modeling work. 


\section{CONTENTS}

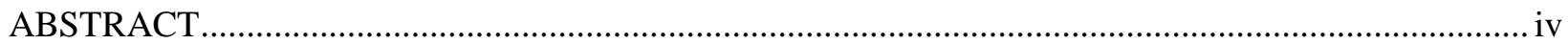

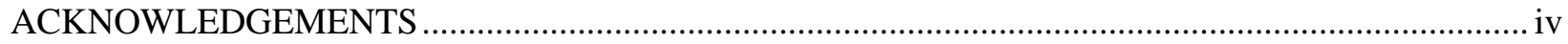

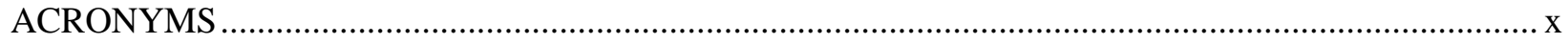

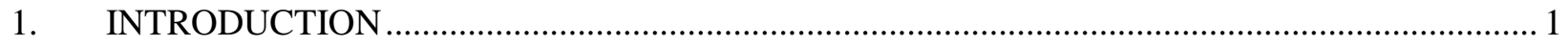

2. CHANNEL GAP MEASUREMENT PROBE THEORY AND DESIGN .................................. 2

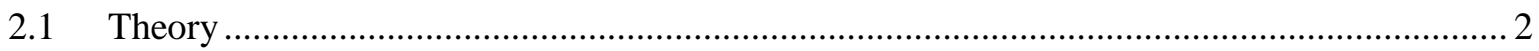

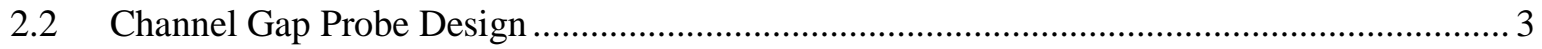

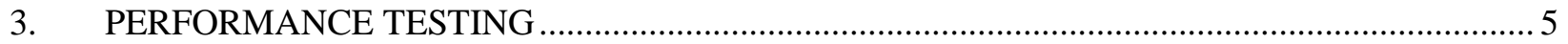

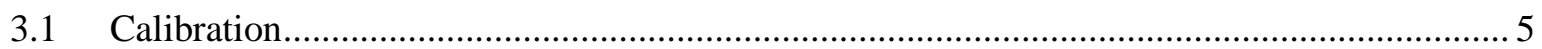

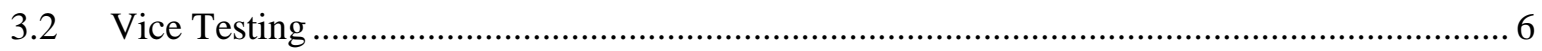

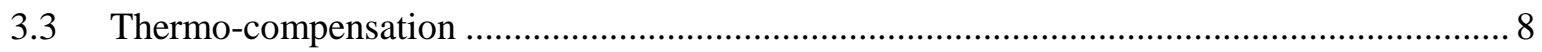

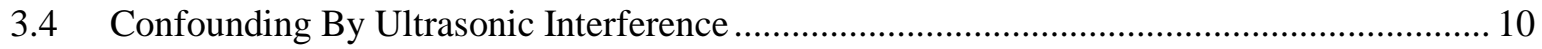

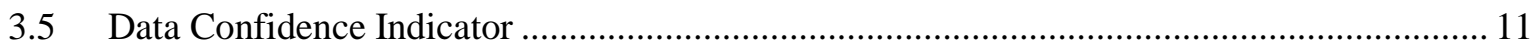

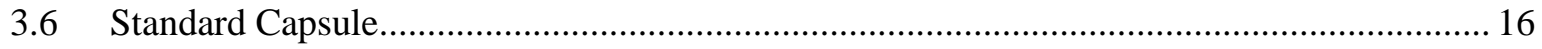

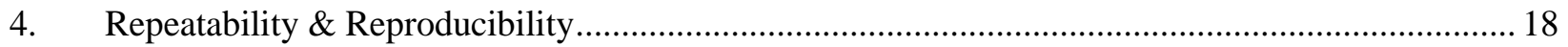

5. REPRESENTATIVE CHANNEL GAP PROBE DATA ........................................................ 21

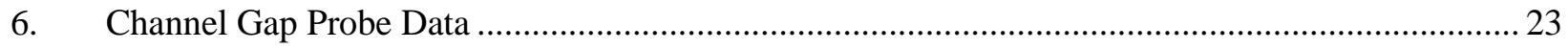

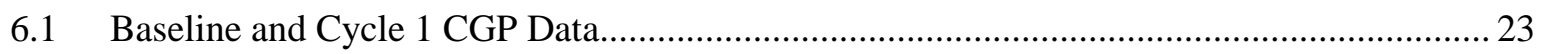

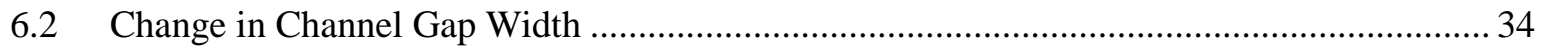

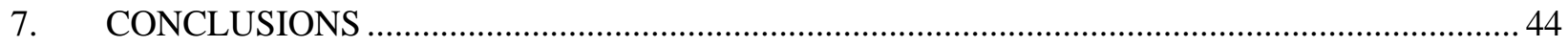

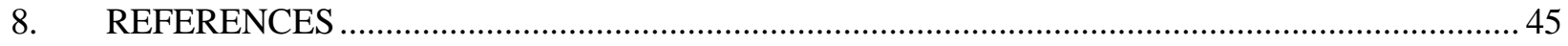

\section{FIGURES}

Figure 1. The EMPIrE capsule has four plates arranged with two levels. ............................................... 1

Figure 2. Visual checks and CGP measurements are performed to check for significant deformation of the plates as shown here for the top plate from the RERTR-12 experiment Visual check for RERTR-12 was performed in a hot cell during PIE.

Figure 3. The TOF measurement is dependent on the distance between the two transducers shown

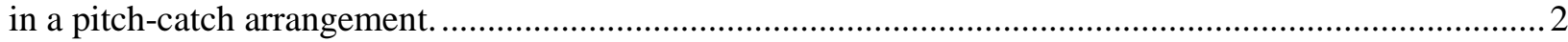

Figure 4. The design of the insertion wand is shown and the two UT transducers are mounted within two leaf springs as displayed. The leaf springs are compressed as the wand is inserted into the channels. 
Figure 5. Close-up of the transducer end of the wand showing the dog bone leaf spring. The transducers are facing each other on the back sides of the leaf springs.................................................. 3

Figure 6. A schematic of the fully assembled channel gap probe is shown.............................................. 4

Figure 7. The CGP is broken up into two sections for ease of storage .................................................. 4

Figure 8. Figure showing how the channel gap probe is used in the ATR canal......................................5

Figure 9. The CGP wand is inserted into the calibration blocks and through the capsule. Date is

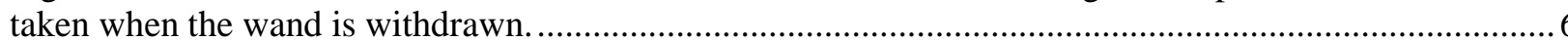

Figure 10. Figure 10 shows how the calibration blocks are constructed and the resulting data from

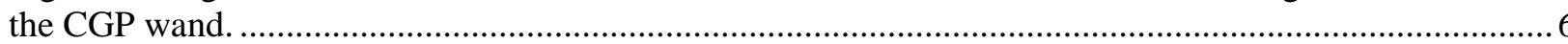

Figure 11. The vice test fixture mounted on the water tank with the wand probe under test and a close-up of the vise test fixture.

Figure 12. Ideally the slope of the line between the measured gap and the actual gap would be 1 . Wand \#114 has a slope close to 1 . Wand \#113 has a slope noticeably less than 1 and the wand has experienced geometric instability as implied by the slope change.

Figure 13. A TC is embedded in the measurement wand above the UT crystals. ..................................... 8

Figure 14. The thermal response for the probe being heated up and then allowed to cool in water. An exponential fit has been made to $2 \mathrm{X}$ the thermal time constant..................................................... 8

Figure 15. The dependence of sound speed in water on temperature is shown. ..................................... 9

Figure 16. The resulting measurement error due to temperature changes in the sonic water path. The reference temperature of the canal water is $20^{\circ} \mathrm{C}$ and the nominal gap width is $4.4 \mathrm{~mm}$ $(0.1730 ”)$.

Figure 17. Despite there being a $2+{ }^{\circ} \mathrm{C}$ temperature differential, the wand scanning rate was slow enough that the maximum temperatures equalized on insertion and withdrawal despite the probe stopping (insertion) and starting (withdrawal) at different temperatures. The insertion graph is generated from right to left and the withdrawal graph is created from left to right.

Figure 18. The sharp increases in measured channel width that have a height of $1 \mathrm{~mm}(0.04$ ") are due to choosing the wrong peak in the signal packet. Note that the sharp increases are very repeatable.

Figure 19. Variation in the Channel Gap measurements can be correlated with sharp changes in the maximum amplitude level.

Figure 20. MP-1wand buckling can cause major changes in the patterns generated by the amplitude data.

Figure 21. Despite the maximum amplitude showing major buckling in the probe, the channel width measurements are largely unaffected. The black data set has the most variation.

Figure 22. Empire wand buckling can cause major changes in the patterns generated by the amplitude data.

Figure 23. The maximum amplitude data shows signs of major buckling in the EMPIrE probe, the channel width measurements are significantly affected with a bias of $0.25 \mathrm{~mm}(0.01$ "). Note that the calibration check side of the plot (right side with distinctive steps) are grouped much closer. Since the calibration steps are of known height, the calibration process forces the steps to be "right" which in turn biases the capsule measurements. 
Figure 24. The CGP system can experience slight miss-registration of the wand position as indicated by the green plot.

Figure 25. The standard capsule is a good indication of the best measurement resolution obtainable by the CGP. The dummy fuel plates appear to have less surface roughness and the dummy plates are rigidly fixed which combine to significantly reduce plate variability.

Figure 26. The signal amplitude varies significantly when the wand transitions into/out of the capsule and at the standard gap transitions in the calibration block. 18

Figure 27. The signal amplitude also varies the most at the major transition locations.

Figure 28. Example graph showing the raw CGP data for channel 2 in the EMPIrE 8 capsule. The plot also shows the valid locations to estimate the measurement statistics.

Figure 29. There were four reproducibility sets and there were at least 3 replicates in each set. The separate reproducibility sets are defined by dates and times when the data files were collected.

Figure 30. The channel gap width (1) and amplitude (r) data for channel 1 within EMPIrE capsule 2.

Figure 31. The channel gap width (l) and amplitude (r) data for channel 2 within EMPIrE capsule 2.

Figure 32. The channel gap width (l) and amplitude (r) data for channel 3 within EMPIrE capsule 2.

Figure 33. The channel gap width (l) and amplitude (r) data for channel 1 within EMPIrE capsule 6.

Figure 34. The channel gap width (l) and amplitude (r) data for channel 2 within EMPIrE capsule 6.

Figure 35. The channel gap width (l) and amplitude (r) data for channel 3 within EMPIrE capsule 6.

Figure 36. Baseline (blue traces) and Cycle 1 (red traces) CGP measurements for capsule EMP-1 with descriptions of the plate types between the plots of channel gap width.

Figure 37. Baseline (blue traces) and Cycle 1 (red traces) CGP measurements for capsule EMP-2 with descriptions of the plate types between the plots of channel gap width. 26

Figure 38. Baseline (blue traces) and Cycle 1 (red traces) CGP measurements for capsule EMP-3 with descriptions of the plate types between the plots of channel gap width.

Figure 39. Baseline (blue traces) and Cycle 1 (red traces) CGP measurements for capsule EMP-4 with descriptions of the plate types between the plots of channel gap width. .28

Figure 40. Baseline (blue traces) and Cycle 1 (red traces) CGP measurements for capsule EMP-5 with descriptions of the plate types between the plots of channel gap width.

Figure 41. Baseline (blue traces) and Cycle 1 (red traces) CGP measurements for capsule EMP-6 with descriptions of the plate types between the plots of channel gap width. .30

Figure 42. Baseline (blue traces) and Cycle 1 (red traces) CGP measurements for capsule EMP-7 with descriptions of the plate types between the plots of channel gap width.

Figure 43. Baseline (blue traces) and Cycle 1 (red traces) CGP measurements for capsule EMP-8 with descriptions of the plate types between the plots of channel gap width. 
Figure 44. CGP measurements for the ATR Dummy capsule that contains four swaged dummy plates.

Figure 45. Red trace is change in channel gap (Cycle 1 data - Baseline data) and blue points represent modeling data from the code PLATE for EMP-1.

Figure 46. Red trace is change in channel gap (Cycle 1 data - Baseline data) and blue points represent modeling data from the code PLATE for EMP-2.

Figure 47. Red trace is change in channel gap (Cycle 1 data - Baseline data) and blue points represent modeling data from the code PLATE for EMP-3.

Figure 48. Red trace is change in channel gap (Cycle 1 data - Baseline data) and blue points represent modeling data from the code PLATE for EMP-4.

Figure 49. Red trace is change in channel gap (Cycle 1 data - Baseline data) and blue points represent modeling data from the code PLATE for EMP-5.

Figure 50. Red trace is change in channel gap (Cycle 1 data - Baseline data) and blue points represent modeling data from the code PLATE for EMP-6.

Figure 51. Red trace is change in channel gap (Cycle 1 data - Baseline data) and blue points represent modeling data from the code PLATE for EMP-7.

Figure 52. Red trace is change in channel gap (Cycle 1 data - Baseline data), blue points represent modeling data from the code PLATE, and green traces through the monolithic plates are from Abaqus modeling for EMP-8.

Figure 53. Change in channel gap of the ATR Dummy capsule runs on April 19, 2018 and April 30, 2018.

Figure A1. Baseline (blue traces) CGP measurements for capsule EMP-9 with descriptions of the plate types between the plots of channel gap width. .46

Figure A2. Baseline (blue traces) CGP measurements for capsule EMP-10 with descriptions of the plate types between the plots of channel gap width.

Figure A3. Baseline (blue traces) CGP measurements for capsule EMP-11 with descriptions of the plate types between the plots of channel gap width. 48

Figure A4. Baseline (blue traces) CGP measurements for capsule EMP-12 with descriptions of the plate types between the plots of channel gap width.

\section{TABLES}

Table 1 . The worst case Reproducibility and Repeatability values taken from the channel gap and UT amplitude profiles.

Table 2. Key for the plate type indicators in CGP data figures. 


\section{ACRONYMS}

ATR Advance Treatment Reactor

CGP Channel Gap Probe

EMPIrE European Mini-Plate Irradiation Experiment

HERACLES Europe's High Performance Research Reactors have teamed up in a common initiative to support the world-wide non-proliferation efforts by minimizing their usage of HEU material.

HEU High Enriched Uranium

INL Idaho National Laboratory

LEU Low Enriched Uranium

M3 Material, Management and Minimization

MP-1 LP Mini-plate experiment Low power

MP-1 MP Mini-plate experiment Medium Power

PIE Post-irradiation examinations

STD Standard of Deviation

TC Thermocouple

TOF Time of Flight

UT Ultrasonic Transducer 


\section{INTRODUCTION}

EMPIrE aims to provide data for the "comprehension phase" of the European Low Enriched Uranium (LEU ) U-Mo Fuel Development program for the conversion of European civilian research reactors. The majority of the fuel performance data will be obtained during post-irradiation examinations (PIE) of the fuel. The primary EMPIrE fuel specimen design is based on uranium-molybdenum (U-Mo) alloy particles dispersed in an aluminum matrix clad in the European alloy AG3NE and in the form of mini-plate sized specimens (1" x 4" plates). Secondary EMPIrE specimens include U-Mo monolithic fuel clad in European alloy AlFeNi. The experiment is designed in such a way that U-Mo samples achieve three distinct end-oflife burnup groupings. Irradiation has taken place at ATR.

The fuel plates are contained in fuel capsules holding four plates each, with 2 plates at 2 levels. Once the capsules with the plates are irradiated, they have to be inspected during the outage in-between cycles. The capsules need to have visual inspection performed and channel gap widths measured prior to reinsertion. These inspections are to ensure that the plates have not deformed significantly. Obstruction of the coolant channels and restriction of the coolant flow are less of a concern in EMPIrE testing as the channels were made intentionally wide. The Channel Gap Probe has been developed to measure channel gap widths, which serves as indirect measures of the fuel plate performance and deformation in the course of irradiation which enables the evaluation of the risk from continuing irradiation prior to reinsertion of the plates in reactor.

The EMPIrE mini-plate experiment is comprised of 48 fuel plates that will be irradiated in ATR to support the development of LEU U-Mo dispersion fuel for application in European research and test reactors. In order for a fuel plate to be inserted into ATR, it must pass a well-defined set of requirements defined in fuel specification (e.g., min-clad, fuel out of zone, homogeneity, ultrasonic testing inspection, etc.). This is to assure the maximum performance of the fuel plates upon irradiation. Candidate fuel plates have been fabricated and passed the respective inspections at AREVA-CERCA and ANL. The INL was also required to perform additional inspection.

Four fuel plates were positioned in the capsules at two levels (Figure 1). After each reactor cycle the plates have to be inspected while in the capsules before reinsertion into the reactor. First visual inspection is performed, followed by the measurement of the channel gap widths, see Figure 2. These inspections are to ensure that the plates have not deformed significantly.

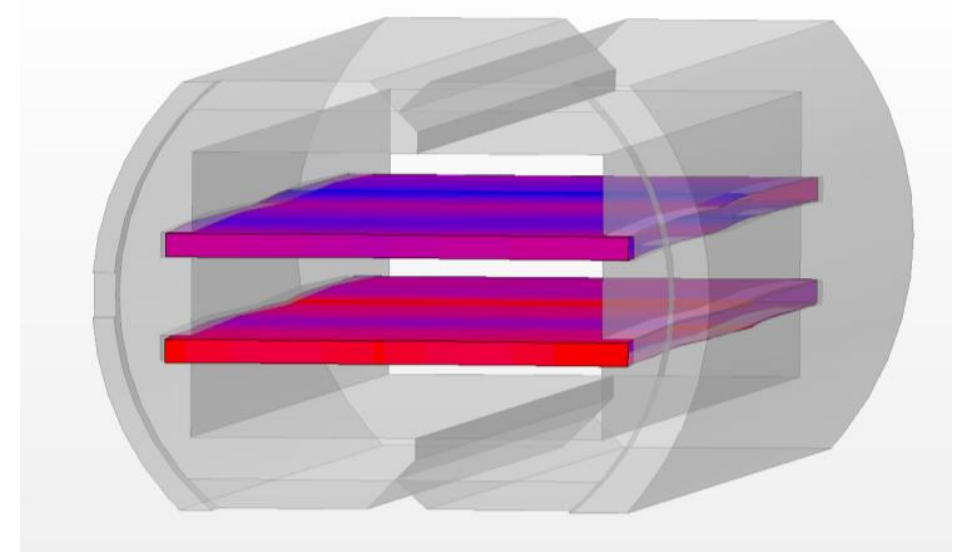

Figure 1. The EMPIrE capsule has four plates arranged with two levels. 


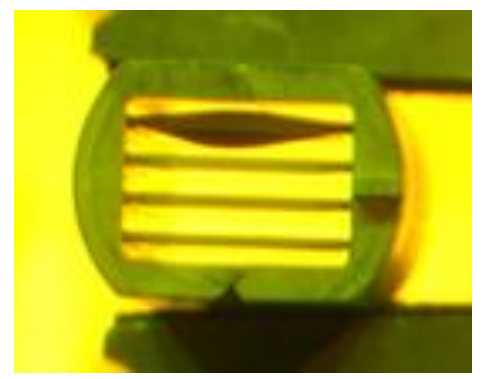

Figure 2. Visual checks and CGP measurements are performed to check for significant deformation of the plates as shown here for the top plate from the RERTR-12 experiment Visual check for RERTR-12 was performed in a hot cell during PIE.

This report will start with the physical basis of the channel gap measurement. The process to ensure the quality of the engineering implementation of the theory will be presented next. Since some of the capabilities in the CGP have been developed after EMPIrE testing combined with the need to make a single document as comprehensive as possible, plates from the MP-1 testing campaign are used to illustrate the newer capabilities. Because the CGP will be characterizing irradiated capsules that are radiating heat, the thermal response of the thermocouple in the probes will be characterized to help access temperature gradient effects on gap measurements. The summary results from a Repeatability and Reproducibility study will be discussed as well as probe performance. The pre-work just described supports the main objectives of the CGP project. The CGP objectives are to develop an effective baseline for each non-irradiated capsule and measure channel width data in irradiated capsules to assess the performance and safety of the fuel plates.

\section{CHANNEL GAP MEASUREMENT PROBE THEORY AND DESIGN 2.1 Theory}

The theory behind the CGP measurement is simplistic and efficiently implementable. The channel gap measurement is based on the time of flight (TOF) measurements of ultrasound travelling from one transducer to another $[1,2]$. This type of transducer arrangement is commonly referred to as a "pitchcatch" arrangement as shown in Figure 3. For the EMPIrE application, the two transducers will be submerged under water. The speed of sound in water as a function of temperature is well characterized, thus the TOF measurements can be converted into distance between the two transducers.

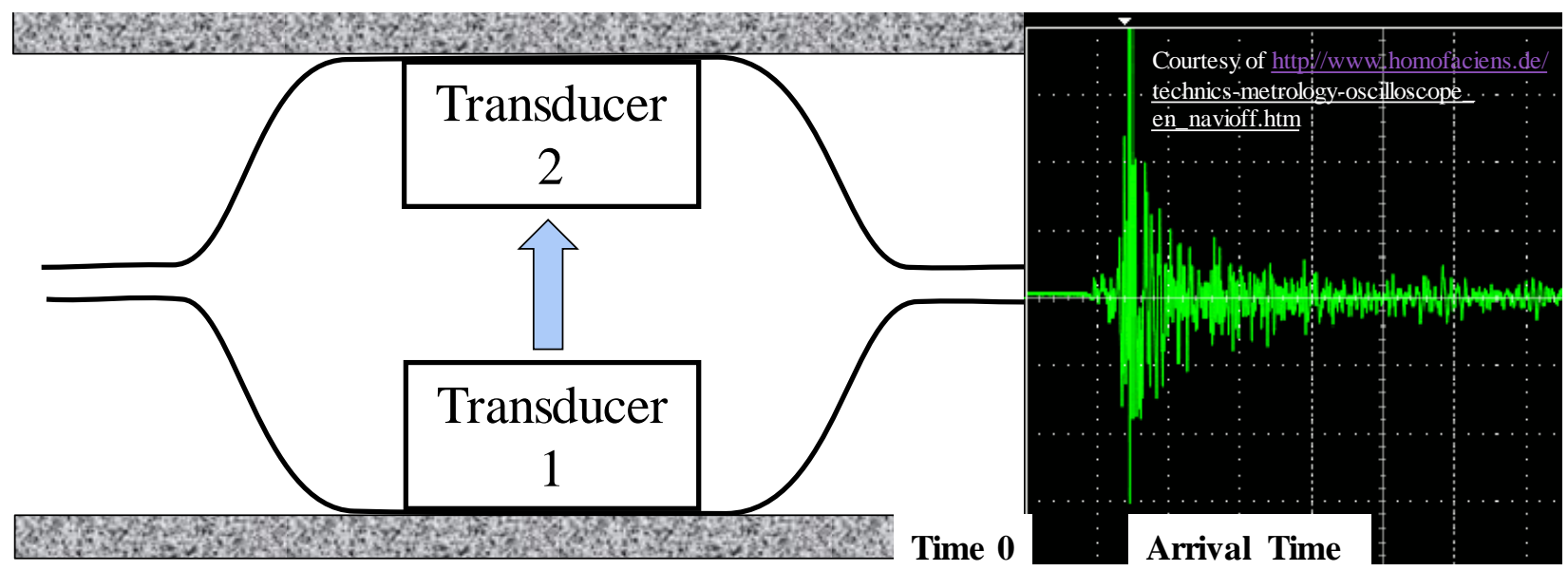

Figure 3. The TOF measurement is dependent on the distance between the two transducers shown in a pitch-catch arrangement. 


\subsection{Channel Gap Probe Design}

A measurement wand is used to insert the Ultrasonic Transducers (UT) into the capsule channels. The measurement wand shown in Figure 4 is constructed from a long thin strip of metal that has two leaf springs at the insertion end. The two UT crystals are affixed to the flat area of the spring and their faces are nominally parallel to each other. Figure 5 shows a close up of the transducer end of the wand. The leaf springs have a dog bone cut out at the proximal end. The dog bone shape allows for more flexibility of the wand to follow the contour of the plates. Also located in the wand is a thermocouple that is $44.5 \mathrm{~mm}$ above the UT crystals. The thermocouple is used to compensate for temperature gradients when the wand is submerged in water.

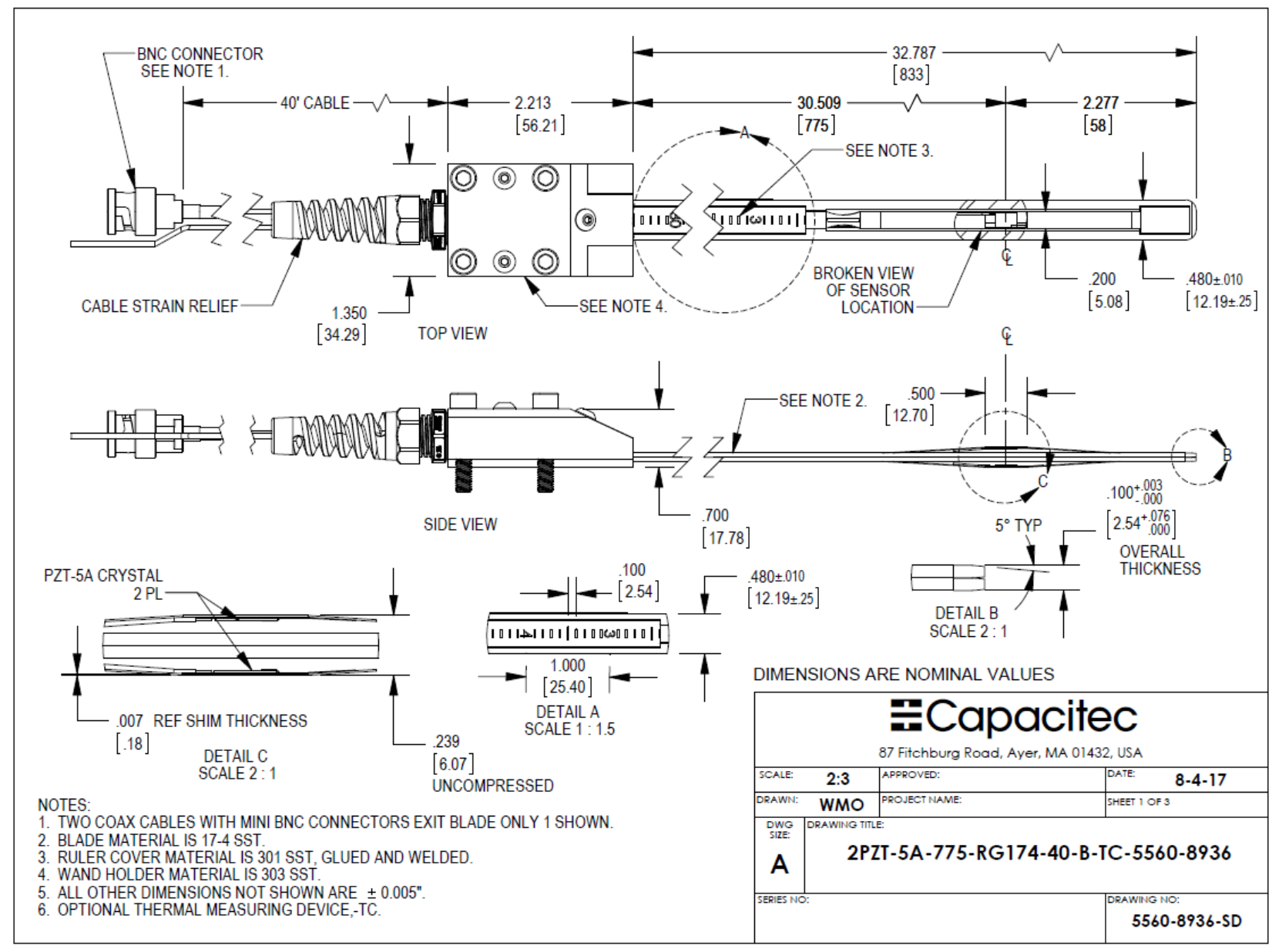

Figure 4. The design of the insertion wand is shown and the two UT transducers are mounted within two leaf springs as displayed. The leaf springs are compressed as the wand is inserted into the channels.

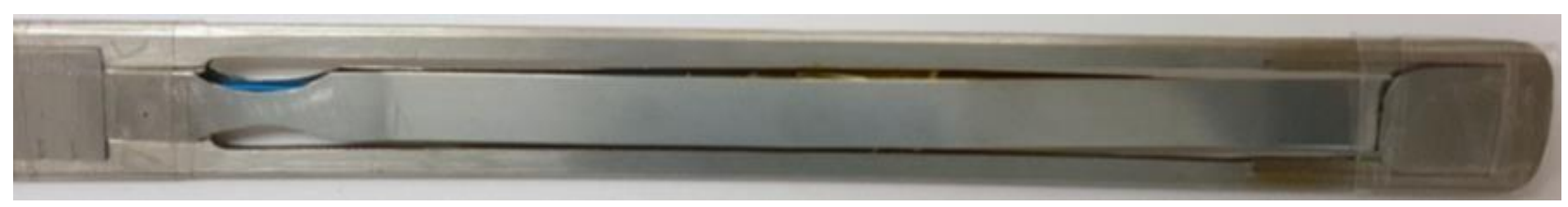

Figure 5. Close-up of the transducer end of the wand showing the dog bone leaf spring. The transducers are facing each other on the back sides of the leaf springs. 
The wand is then connected to the CGP frame. The full frame is shown in Figure 6. The key features of the frame are a motor and a slide to insert the wand, an indexing carriage to choose the channel and a bell shaped guide to insert the capsule into the probe. Since the CGP system is for short-term use at ATR, the frame is designed to be broken down into two parts as shown in Figure 7 and is stored in a $2.4 \mathrm{~m}$ case.

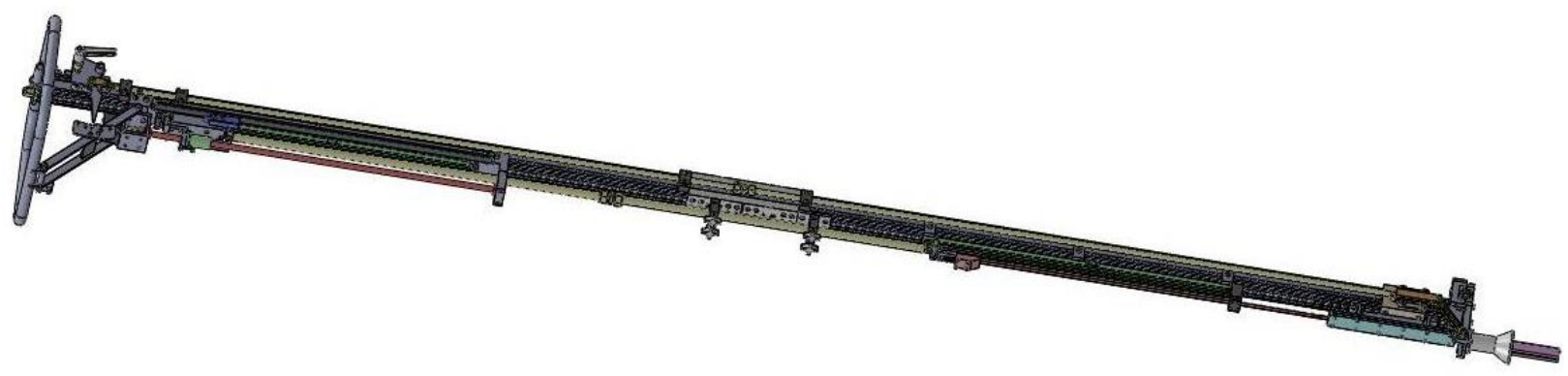

Figure 6. A schematic of the fully assembled channel gap probe is shown.
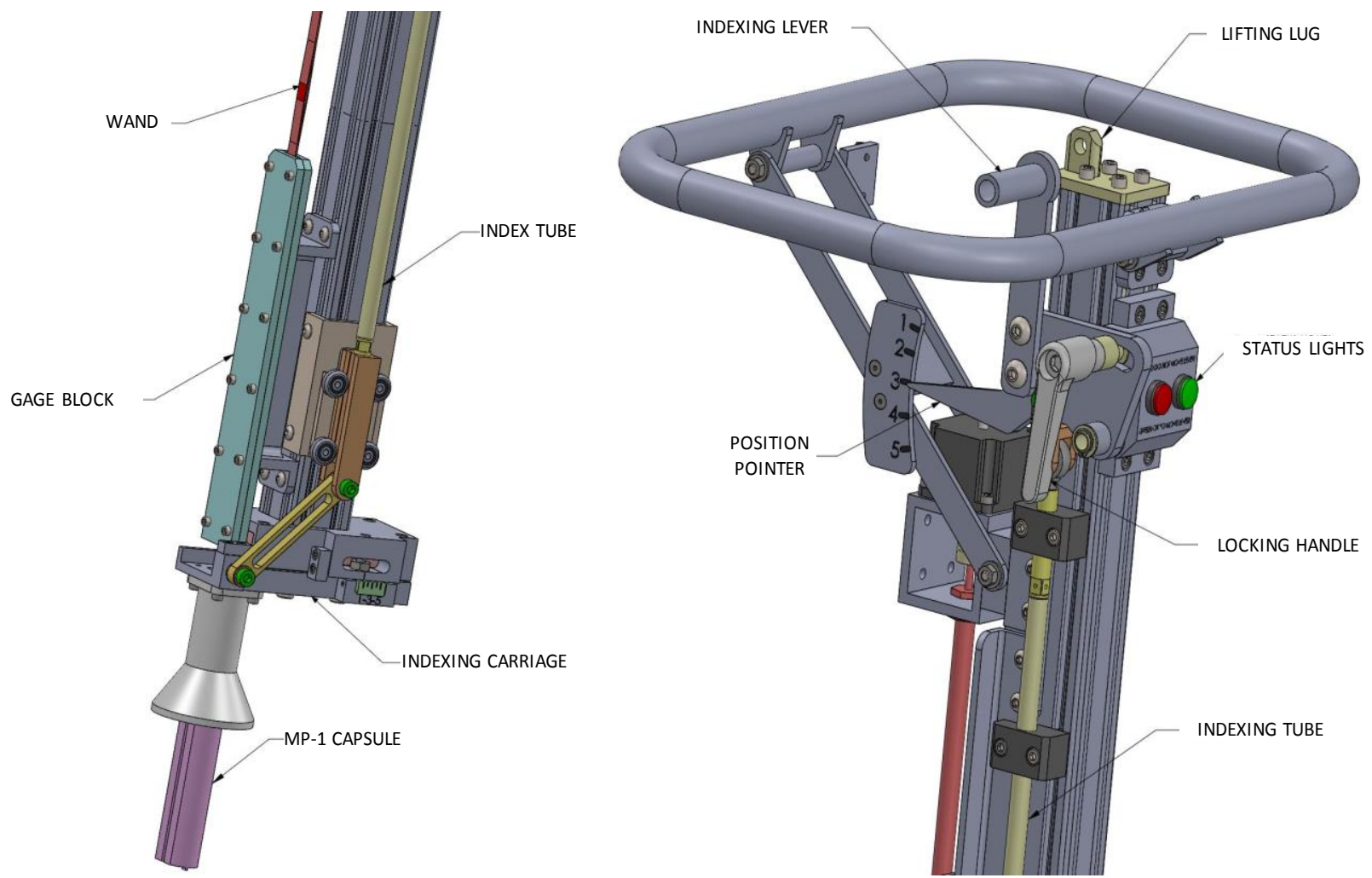

Figure 7. The CGP is broken up into two sections for ease of storage.

Figure 8 indicates how the CGP is used in the ATR canal. A CGP insert is placed on top of a working tray in the canal. The CGP insert has two capsule grippers that hold the capsules. The canal operator places the CGP over the capsule with assistance from a crane. The CGP is then lowered on to the capsule. The desired capsule channel is selected via the indexing lever. Once the CGP is properly set in place and indexed, data collection is performed by the experimental team. 


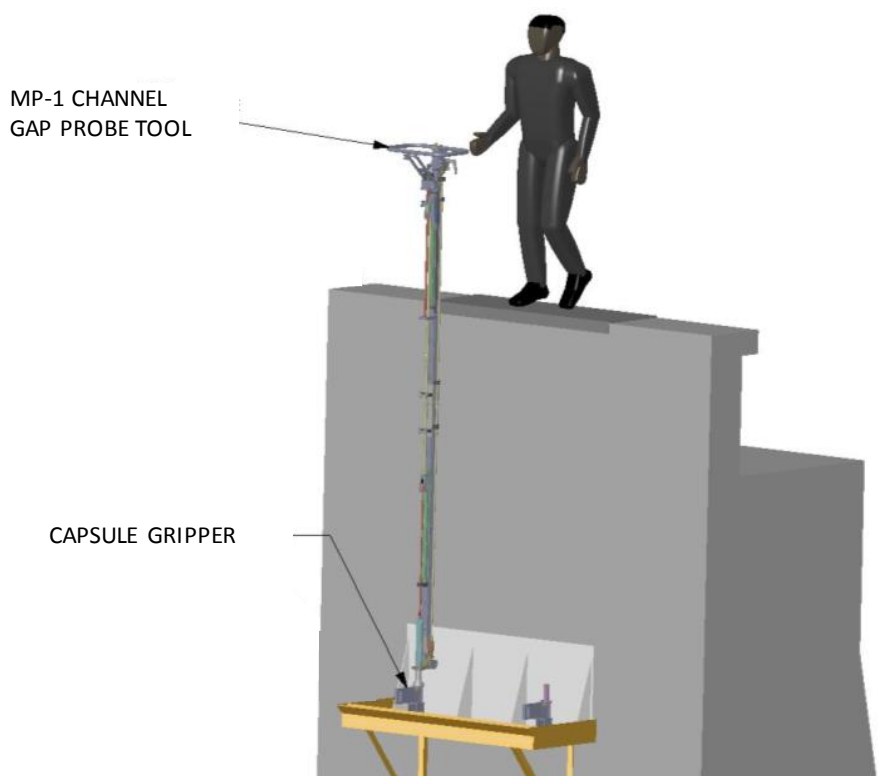

Figure 8. Figure showing how the channel gap probe is used in the ATR canal.

The probe is designed to be used and stored at ATR. The components that make up the CGP are modular and easily replaceable. Probe length change is possible by the replacement of the mast and two tubes which allows flexibility in plate size. The CGP can be quickly broken down and stored in an eight foot container.

\section{PERFORMANCE TESTING 3.1 Calibration}

The first step when performance testing an instrument under various test conditions is to calibrate the sensors. For the channel gap probe, a gauge block is used as shown in Figure 9. The gauge block is used to both calibrate the sensor wand and check its calibration. The actual calibration, calibration check and data collection occurs after the wand is inserted through the calibration block and the capsule. Data collection occurs when the probe is withdrawn as the metal strip needs to be stiffened by tension to prevent buckling during the measurement. Figure 10 shows how the calibration blocks are constructed and the resulting data from the CGP wand. The calibration block widths are measured by the INL calibration lab using a special measurement jig. The data from CGP calibration check is for channel gap widths of $4.7 \mathrm{~mm}(0.186 "), 3.96 \mathrm{~mm}(0.156 ")$ and $3.2 \mathrm{~mm}(0.126$ "). The maximum and the minimum thicknesses are chosen to encompass the expected range of the channel gap measurements for EMPIrE testing.

The CGP wand is calibrated at the beginning of an experiment series. The calibration is checked each time the wand is withdrawn. If any one of the block widths are off by more than $0.254 \mathrm{~mm}(0.001$ "), the CGP wand is recalibrated. The usual cause for the probe going out of calibration is temperature change in the water. The CGP wand does contain a TC that is located $44.45 \mathrm{~mm}$ above the UT crystals. The function of wave speed in water with respect to temperature is well known and temperature changes can be compensated for but the probe can still drift off of calibration. Plastic deformation in the probe's leaf springs also has caused shifts in the calibration by permanently changing probe geometry. 


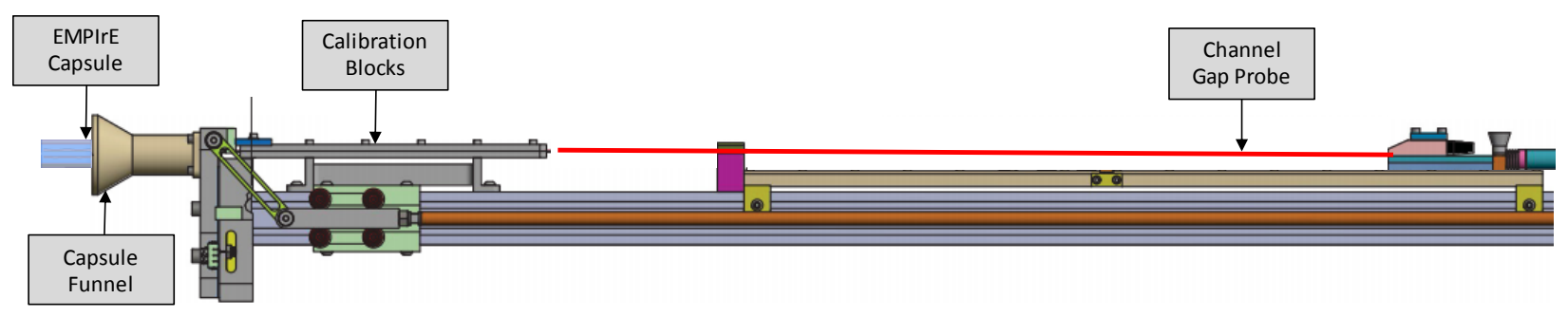

Figure 9. The CGP wand is inserted into the calibration blocks and through the capsule. Date is taken when the wand is withdrawn.
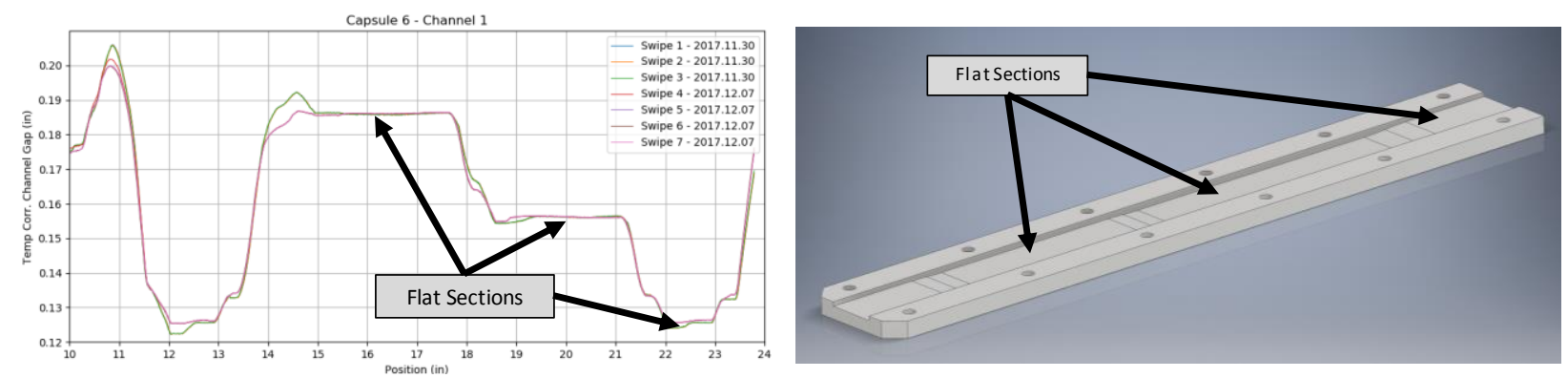

Figure 10. Figure 10 shows how the calibration blocks are constructed and the resulting data from the CGP wand.

\subsection{Vice Testing}

When a new sensor wand is received from the vendor, the wand is tested and qualified using a vice test [3, section 8]. The vice test is designed to identify abnormal behavior and the linear range for the wand over the expected range of channel widths. The amplitude is also monitored for abnormal behavior. Amplitude characterization is important since it can identify these two salient considerations: health of the UT transducers and alignment of the transducers. The signal amplitude has a second order effect on the measured gap width. If the geometry of the wand springs and transducers are appropriate, the slope of the measured channel width with respect to the actual channel width will be 1 . Since the transducers are on the inside of the leaf springs the offsets will be non-zero.

Figure 11 shows the vice test set-up with a close up of the vice fixture. The vice testing characterization process is described in [3] within the Performing Vice Test Measurements section. It has been noted that when a probe undergoes plastic deformation of a spring (tin canning), the vice test shows noticeable change in the resulting data as shown in Figure 12 for the 113 wand. 


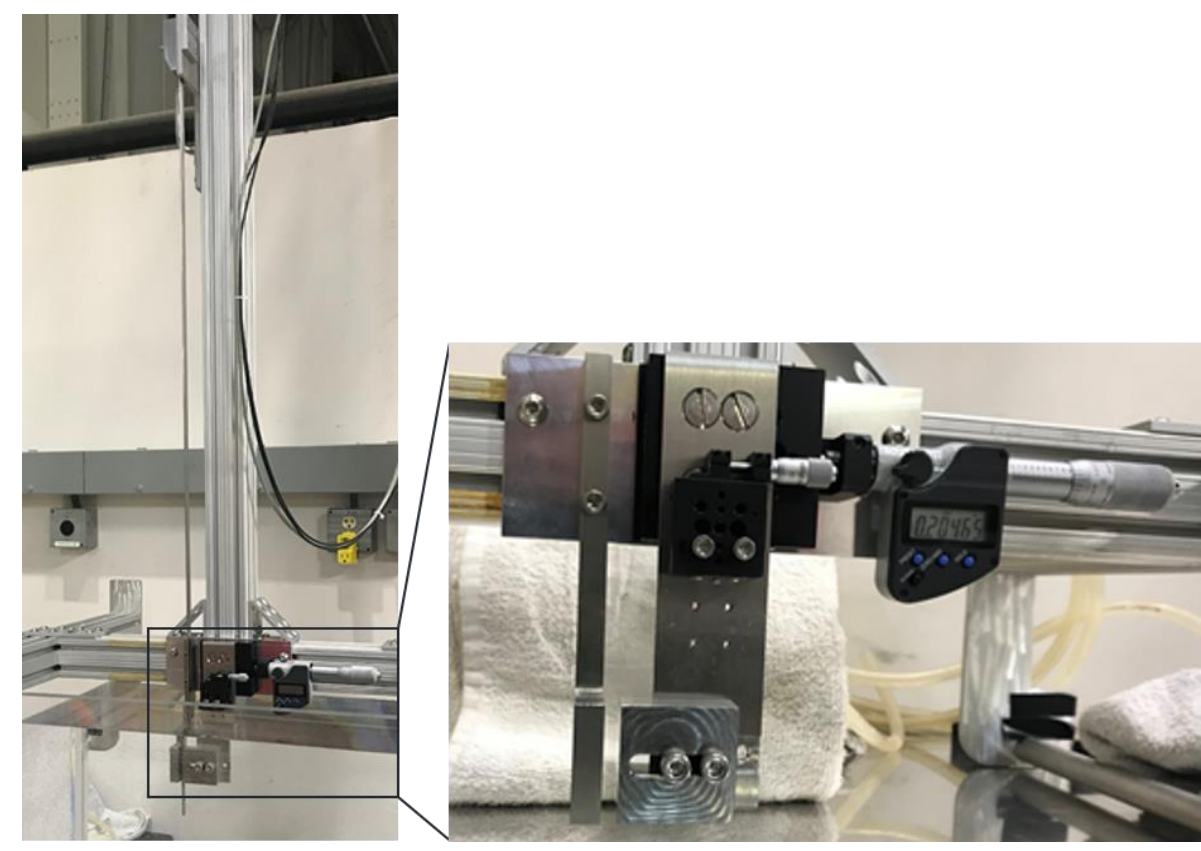

Figure 11. The vice test fixture mounted on the water tank with the wand probe under test and a close-up of the vise test fixture.

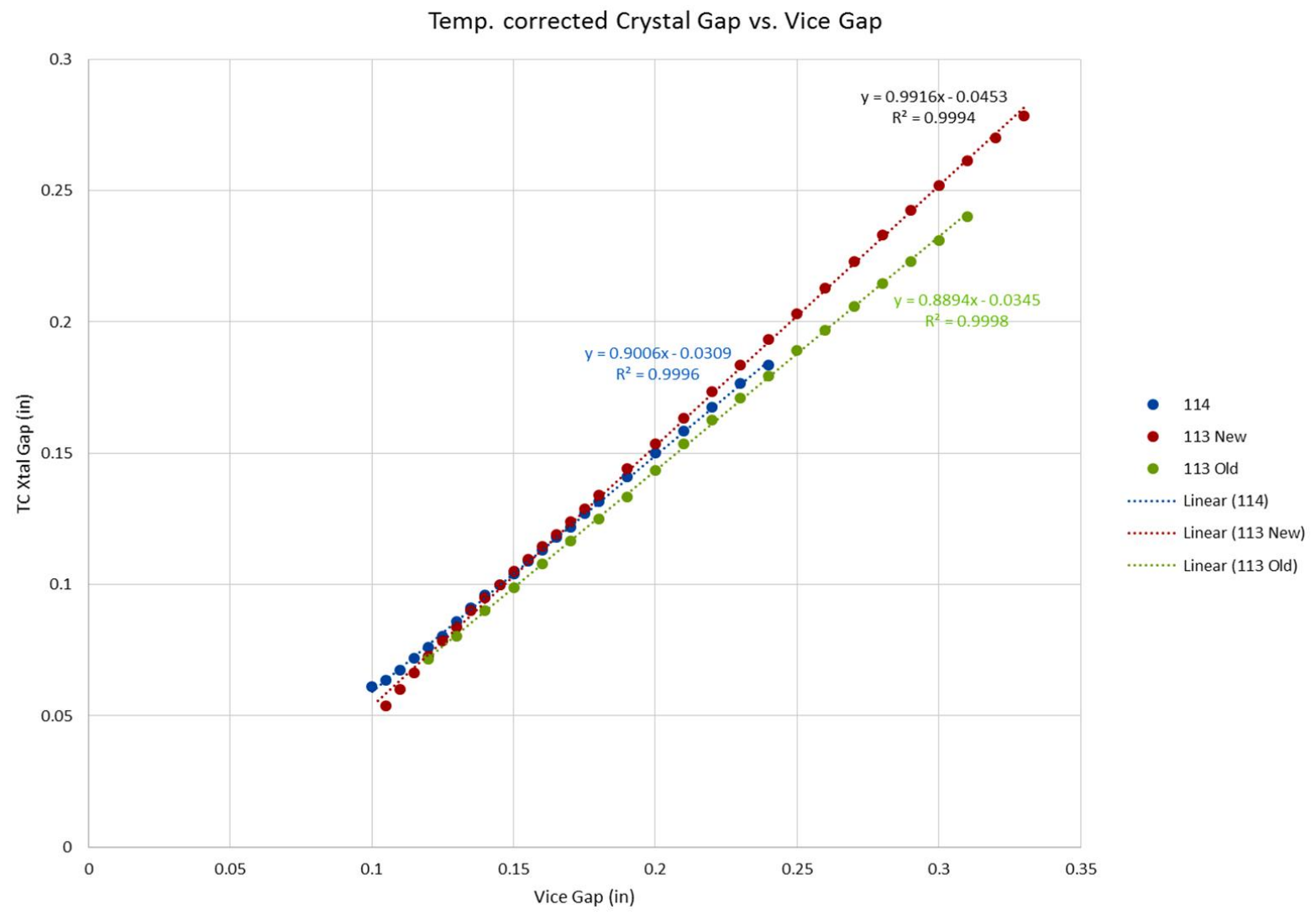

Figure 12. Ideally the slope of the line between the measured gap and the actual gap would be 1 . Wand \#114 has a slope close to 1 . Wand \#113 has a slope noticeably less than 1 and the wand has experienced geometric instability as implied by the slope change. 


\subsection{Thermo-compensation}

As mentioned previously, temperature changes in the water path and on the plate surfaces will affect the gap thickness measurements. To provide a compensation mechanism, a thermocouple (TC) has been embedded $44.5 \mathrm{~mm}$ (1.75") above the UT crystals as shown in Figure 13. Because the wand is moving and the temperature gradients may be dynamic, the thermal response time of the TC needs to be assessed. Twice the thermal time constant (time at which original temperature is reduced by $86.6 \%$ ) [6] is used as the time decay metric.

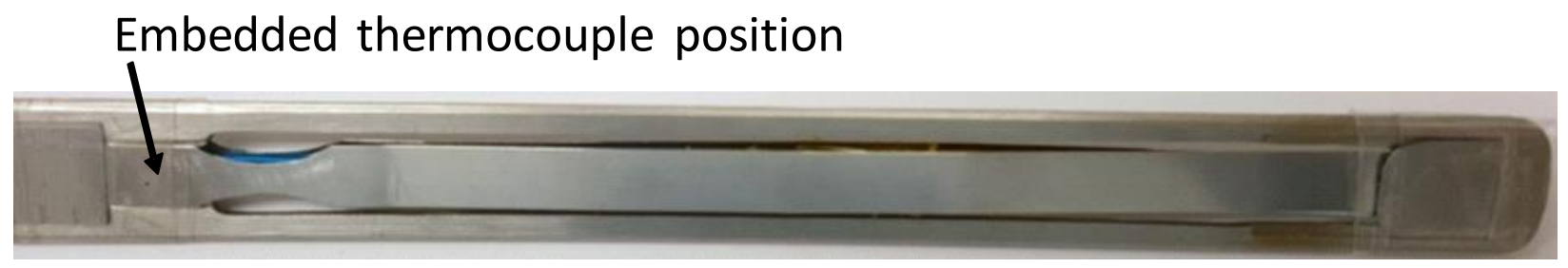

Figure 13. A TC is embedded in the measurement wand above the UT crystals.

An experiment was run where the wand was heated up using a heat gun. The wand was then left to cool in water and air. Newton's law of cooling was used to calculate twice the thermal time constant in air and in water. The resulting thermal decay plot for water and the calculated $2 \mathrm{X}$ thermal decay time $(1.8 \mathrm{~s})$ are shown for water in Figure 14. The decay time for air (3.1 s) is considerably longer than for water as expected.

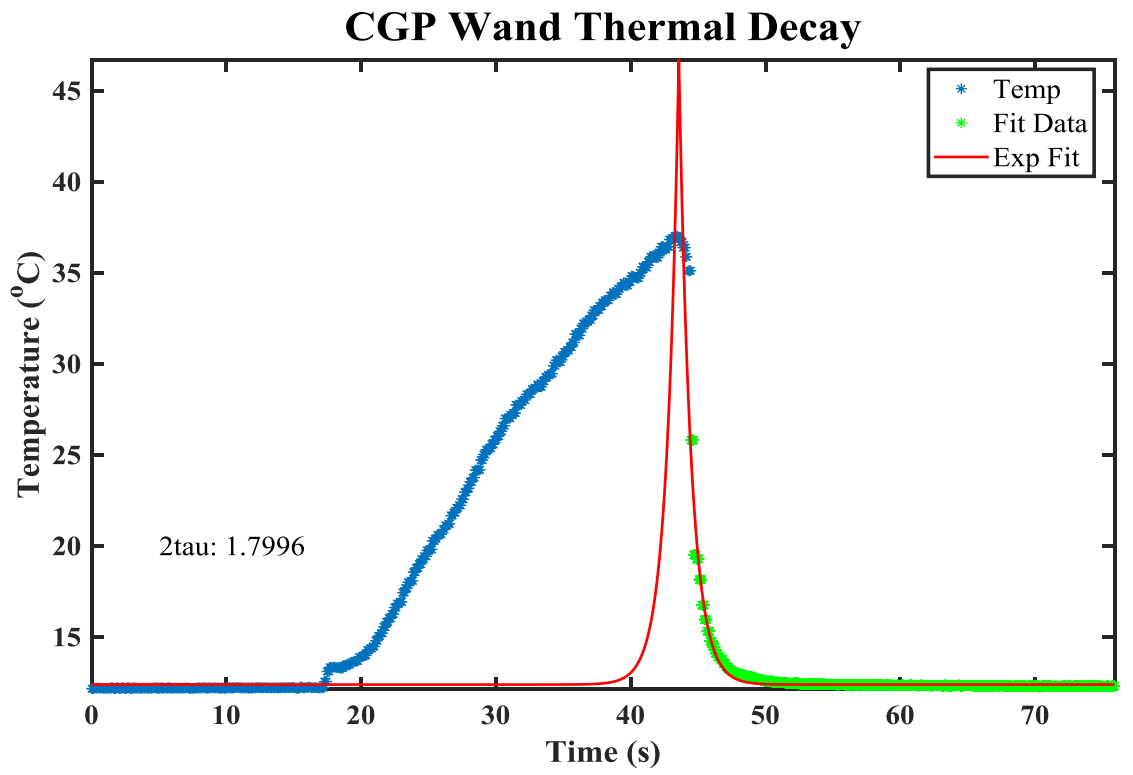

Figure 14. The thermal response for the probe being heated up and then allowed to cool in water. An exponential fit has been made to $2 \mathrm{X}$ the thermal time constant.

The severity of the inaccuracy in the gap width measurements with temperature is a function of the dependence of sound speed in water with temperature. Figure 15 is a plot of wave speed in water with respect to temperature using the Bilaniuk and Wong model [7]. Note that the sound speed sensitivity is maximum closer to the freezing point and minimum close to $75^{\circ} \mathrm{C}$. If water in the channels happened to be near $75^{\circ} \mathrm{C}$, temperature correction would not be necessary. Unfortunately water within the calibration blocks will still be at the nominal canal water temperature. Thus temperature measurements and compensation are still necessary. 


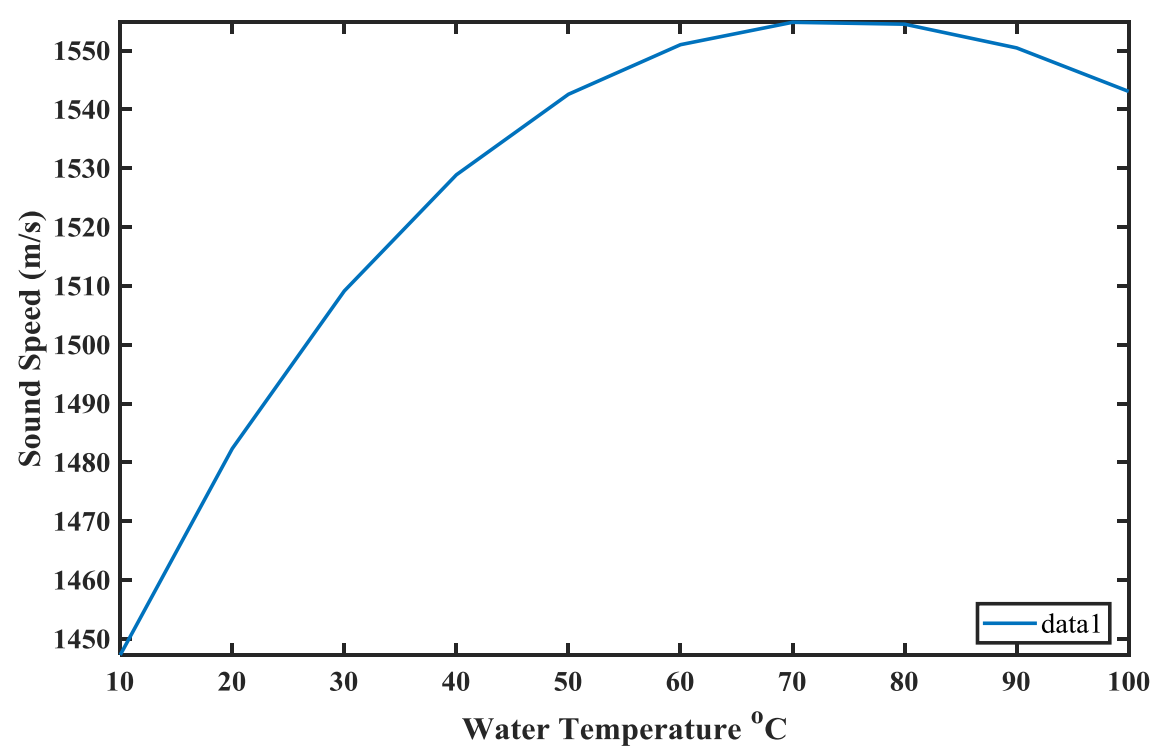

Figure 15. The dependence of sound speed in water on temperature is shown.

The translation of variable sound speed into measurement error is shown in Figure 16. The reference temperature of the canal water is $20^{\circ} \mathrm{C}$ which is close to the nominal temperature of the canal (temperature is seasonal) and the nominal gap width for EMPIrE plates is $4.4 \mathrm{~mm}(0.1730$ "). The CGP system design requirement is for the measurement error to be less than $\pm 0.051 \mathrm{~mm}(0.002$ "). For the water and EMPIrE plate geometry assumed for Figure 16, the change in temperature must be less than $\pm 6^{\circ} \mathrm{C}$ to be within tolerance of $0.051 \mathrm{~mm}$.

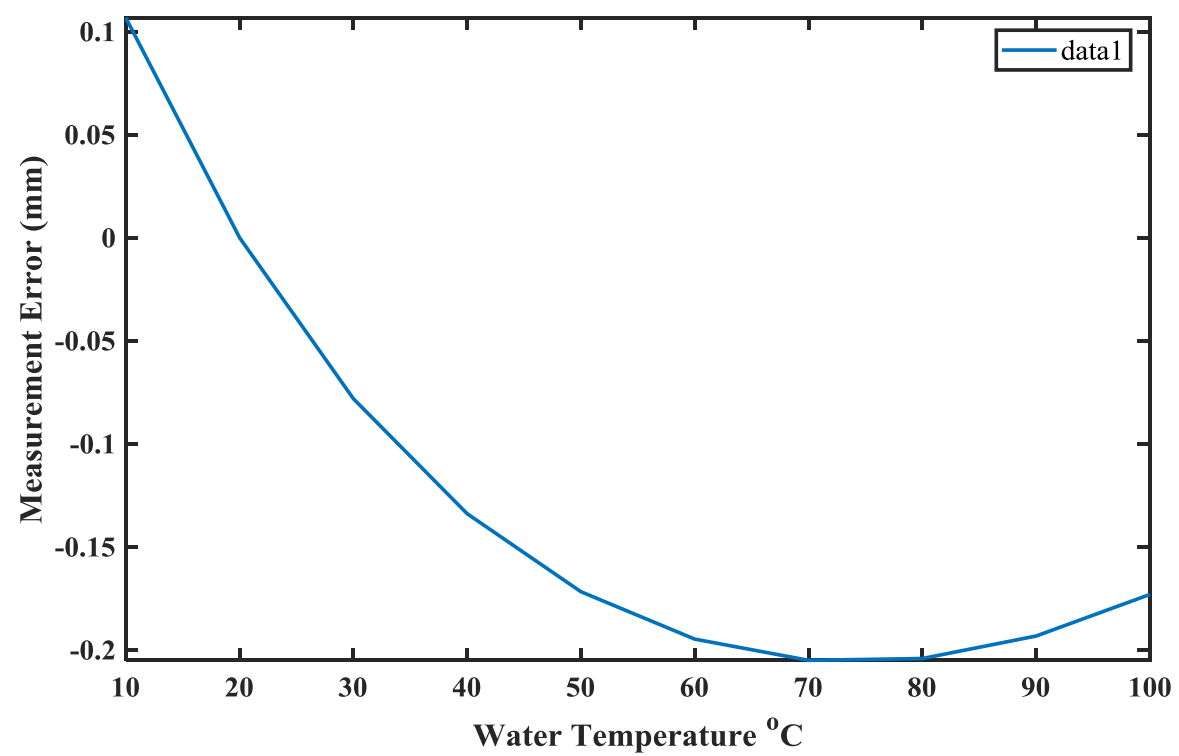

Figure 16. The resulting measurement error due to temperature changes in the sonic water path. The reference temperature of the canal water is $20^{\circ} \mathrm{C}$ and the nominal gap width is $4.4 \mathrm{~mm}(0.1730$ ")

The only temperature compensation mechanism available is time. The decay time of the probe in combination with the maximum allowed temperature change is a starting estimate as to how long or slow the CGP scanning should be. The rule of thumb that is used to collect data for the channel gap probe is that a temperature differential of $>3^{\circ} \mathrm{C}$ (conservative) needs to be seen in a scan before compensatory 
action is taken. At this point the probe scan speed is slowed until the difference between the wand insertion maximum temperature and the maximum withdrawal temperature is less than $3^{\circ} \mathrm{C}$. The maximum temperature differences between the two wand directions will not work because the wand on the way in is generally heating up and the wand on the way out is generally cooling down. As you can see from Figure 14 there is a big difference between the heating and cooling rates. Figure 17 shows this temperature measurement phenomenon for irradiated MP-1 LP plates. The temperature difference in all of the irradiated EMPIrE capsules did not go above $3^{\circ} \mathrm{C}$ therefore corrective action was not warranted during the EMPIrE CGP measurement campaign.

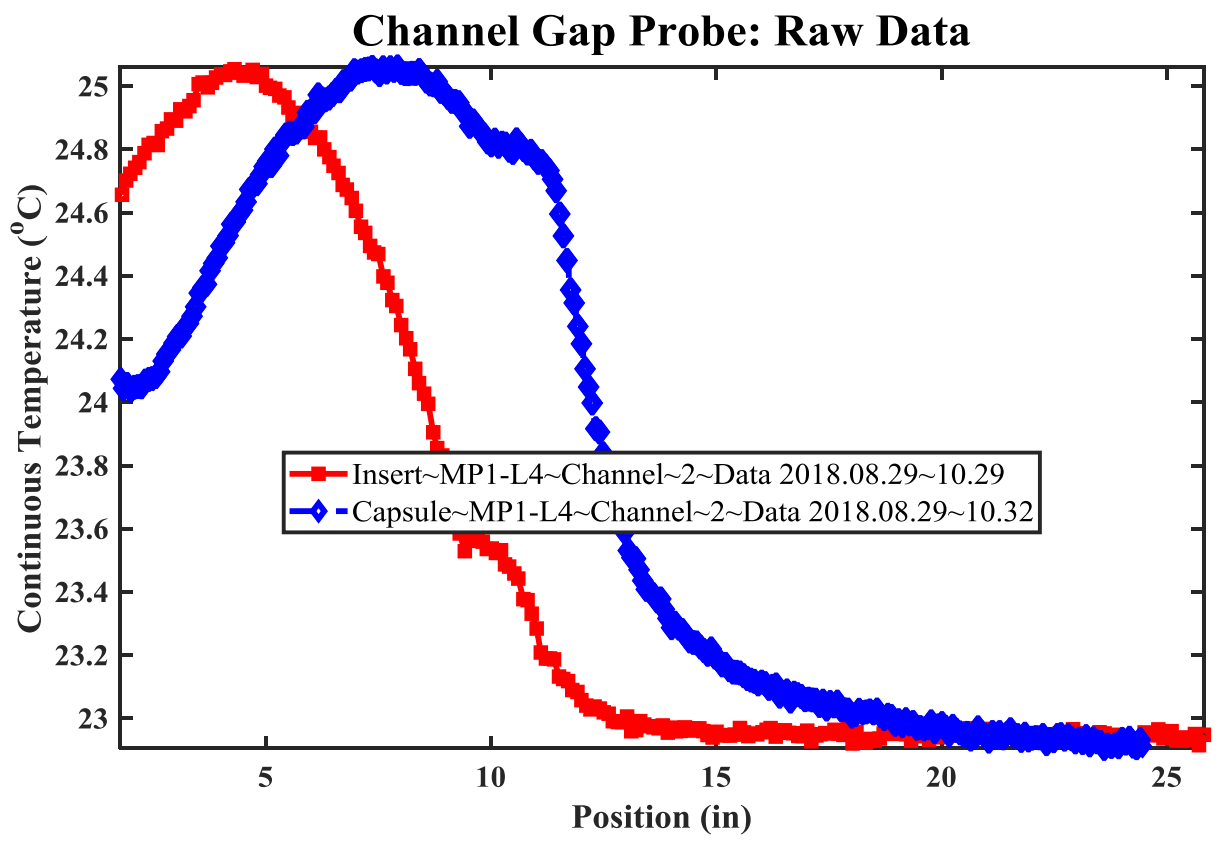

Figure 17. Despite there being a $2+{ }^{\circ} \mathrm{C}$ temperature differential, the wand scanning rate was slow enough that the maximum temperatures equalized on insertion and withdrawal despite the probe stopping (insertion) and starting (withdrawal) at different temperatures. The insertion graph is generated from right to left and the withdrawal graph is created from left to right.

The exact temperature of the water between the crystals is unknown since the TC is $44.5 \mathrm{~mm}$ away from the crystal center. The finite thermal time constant also causes additional uncertainty in the measurements. Thus temperature compensation even with reduced wand scan rates does not guarantee accuracy but it does ensure better measurement repeatability and should reduce the measurement error as well.

\subsection{Confounding By Ultrasonic Interference}

All of the channel gap probes experience interference noise in the ultrasonic signal. The interference noise is most likely coming from sonic reverberations between the two crystals in the wand leaf springs. Some of the noise may also be from near field effects. The sound travel path between the compressed springs is small compared to the transducer diameter. Thus in the near field the ultrasonic beam is not fully formed and sonic energy from the crystal edges interfere with the energy from the crystal center. The reverberation or near field interferences cause the amplitude of received signal to vary and distort. The time of flight measurements are based on the arrival of the first peak that crosses a voltage threshold in the received signal. There are multiple peaks in the received signal packet. Generally, the threshold is set high enough to reject background noise and low enough to capture the first peak (either positive or negative). The sonic interferences may cause the first peak to essentially disappear into the noise. The 
magnitude of the interferences is dependent on the interaction of the plate geometry and the probe. The dynamic nature of the interferences while the probe is moving and changing thermal gradients in the channels compound the issue. The peaks that arrive at a later time in the signal packet can be larger in amplitude and hence trigger the TOF calculation. Although TOF calculations can be performed on any peak in the signal train, the choice of the peak has to be consistent throughout the measurement to be valid. The only way to minimize the effect of interference is to carefully choose the threshold limit on the most stable peak. By making sure the threshold is large enough so that a prior peak does not surpass the threshold and the if desired peak remains large enough to trip the threshold, the TOF measurement can be accurately made. There is no guarantee that the desired peak will always be chosen.

Peak confusion has been documented at the manufacturer of the wand, Capacitec, and at the INL. Peak confusion was not noted in the EMPIrE measurements but it has been noted in some of the narrower channels of MP-1 plates. The smaller spring separation in MP-1capsules amplifies both the multiple UT reverberations between springs and the near field transducer effects. Figure 18, is an example when some of the peaks used to measure TOF were incorrectly chosen. In general, the original peak may be missed and the next later peak in the signal packet is used. The difference in measured channel gap width is noted to be $0.762 \mathrm{~mm}(0.03$ ") at Capacitec and $1.0 \mathrm{~mm}(0.04$ ") at the INL. Thus far, peak confusion has been limited to later arriving peaks which cause a sharp increase in channel width of uniform height. It also may be possible to skip multiple peaks or pick a closer peak. This would cause larger step increases as well as negative going spikes.

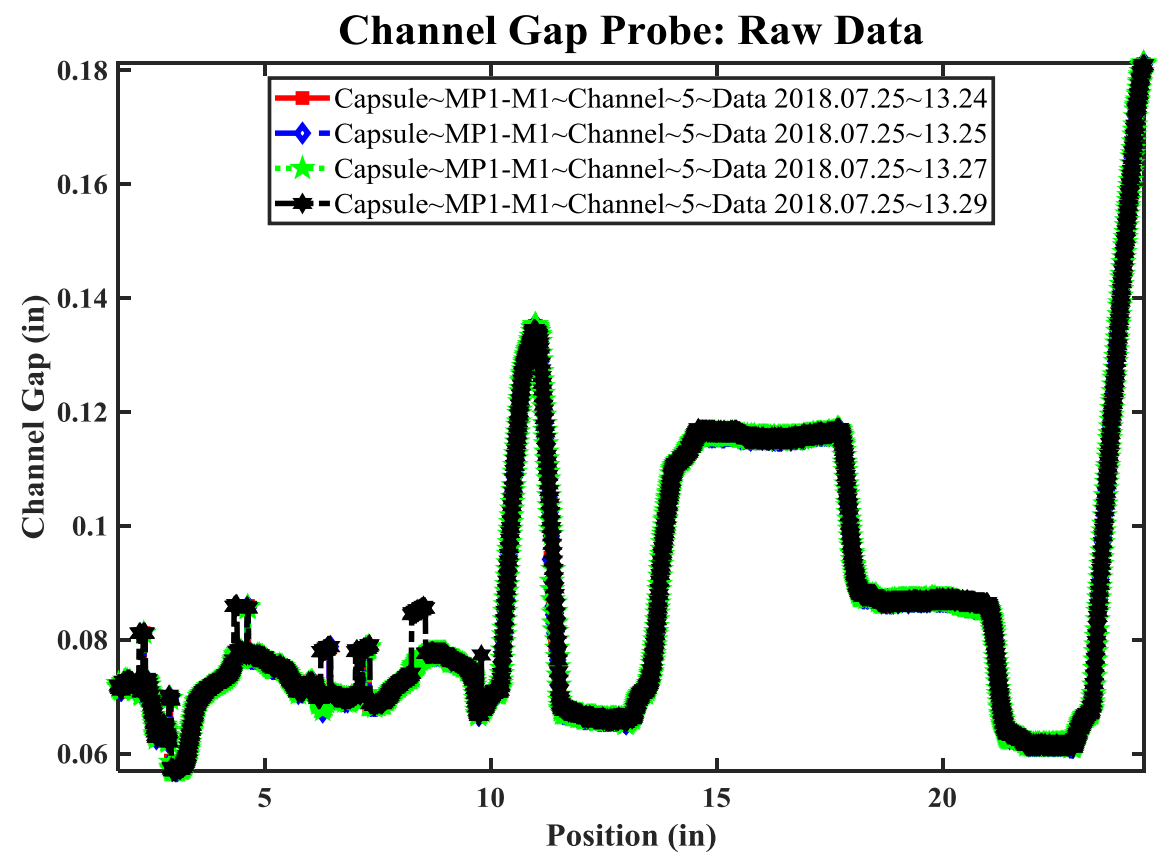

Figure 18. The sharp increases in measured channel width that have a height of $1 \mathrm{~mm}(0.04$ ") are due to choosing the wrong peak in the signal packet. Note that the sharp increases are very repeatable.

\subsection{Data Confidence Indicator}

A concern common to all measurement devices is how does the user ensure the data is valid? This is certainly true since the CGP measurement is influenced by signal interferences and crystal alignment in the wand (see geometry shown in Figure 3). Changes in the signal peak amplitude does have an effect on the resulting measurement. By monitoring the maximum amplitude of the selected peak in the signal packet, confidence in the data can be estimated. Monitoring the amplitude of the ultrasonic peak also provides a health monitoring capability for the ultrasonic crystals. As the crystals age or degrade with 
radiation dose, the ultrasonic output will diminish. If either of the crystals separate from the leaf springs, the amplitude will quickly drop and disappear, if a crystal completely separates. Monitoring the amplitude of the received ultrasonic peak provides an effective indication of the data confidence and transducer health.

Typical CGP channel data are shown in Figure 19. The figure shows the gap width measurements above the peak amplitude measurements for the three channels in the EMPIrE-4 capsule. The channel gap width and amplitude data are repeatable. The data shown in Figure 19 is representative of the EMPIrE results. Channel 1 tends to have the most variation followed by Channel 3. Channel 2 tends to have the least amount of variation. From Figure 19 it can be noted that when there is a significant "bump" in the channel gap measurements, there is a corresponding bump in the amplitude. Both the channel width and amplitude data for Channel 2 are smoother than for the other two channels and all the graphs share the same nominal features for the respective measurement. If the nominal feature shapes change during the measurement campaign, this is an indication that the probe is changing geometry and the situation needs to be assessed. A large amplitude variation in the data warns the user that the data collected in that area is suspect and should be scrutinized. Large amplitude variations usually occur when the probe transitions onto a plate, between plates and off a plate. The amplitude information only provides an indication of the confidence level that the data has been taken under stable conditions and if further inquiry about the data is warranted. The confidence indication from the amplitude data does not accurately predict the accuracy or inaccuracy of the data as will be discussed below.

The peak amplitude information is a sensitive indication of the condition of the CGP sensor wand as shown in Figure 20. The data is from the MP1 baseline series. The wand data started out with an initial data pattern (red, green). The dark-blue graph started out with a big loss in amplitude and a different data pattern. Halfway through the scan (dark blue), the wand made a correction and rejoined the initial data pattern. Note that the dark-blue scan occurred between the red and green scans. After the green scan the, data scan pattern changed again but it appears to be in a new stabilized state. The current state has a smaller amplitude even after a gain increase from 10 to $18 \mathrm{~dB}$ for MP-1 LP L8 through L12 compared to L7 Chan 5 at $18 \mathrm{~dB}$. The wand baseline shift occurred in exactly the second half of the MP1 Capsules 712 campaign. The measurement difference between the two wand states is at least $0.025 \mathrm{~mm}$ (001"). This behavior is consistent with plastic deformation (buckling or tin can effect) of the leaf-springs in the wand. Despite all the variation in the amplitude data, there is very little change in the measured channel width, see Figure 21. Despite the black and light blue data being plotted on top of each other only the black channel gap data is noticeably deviated from the rest of the plots. Also, it is unclear as to what is the cause of the sharp downward spikes in Figure 20. The spikes do not seem to appreciably affect the channel gap measurements. 

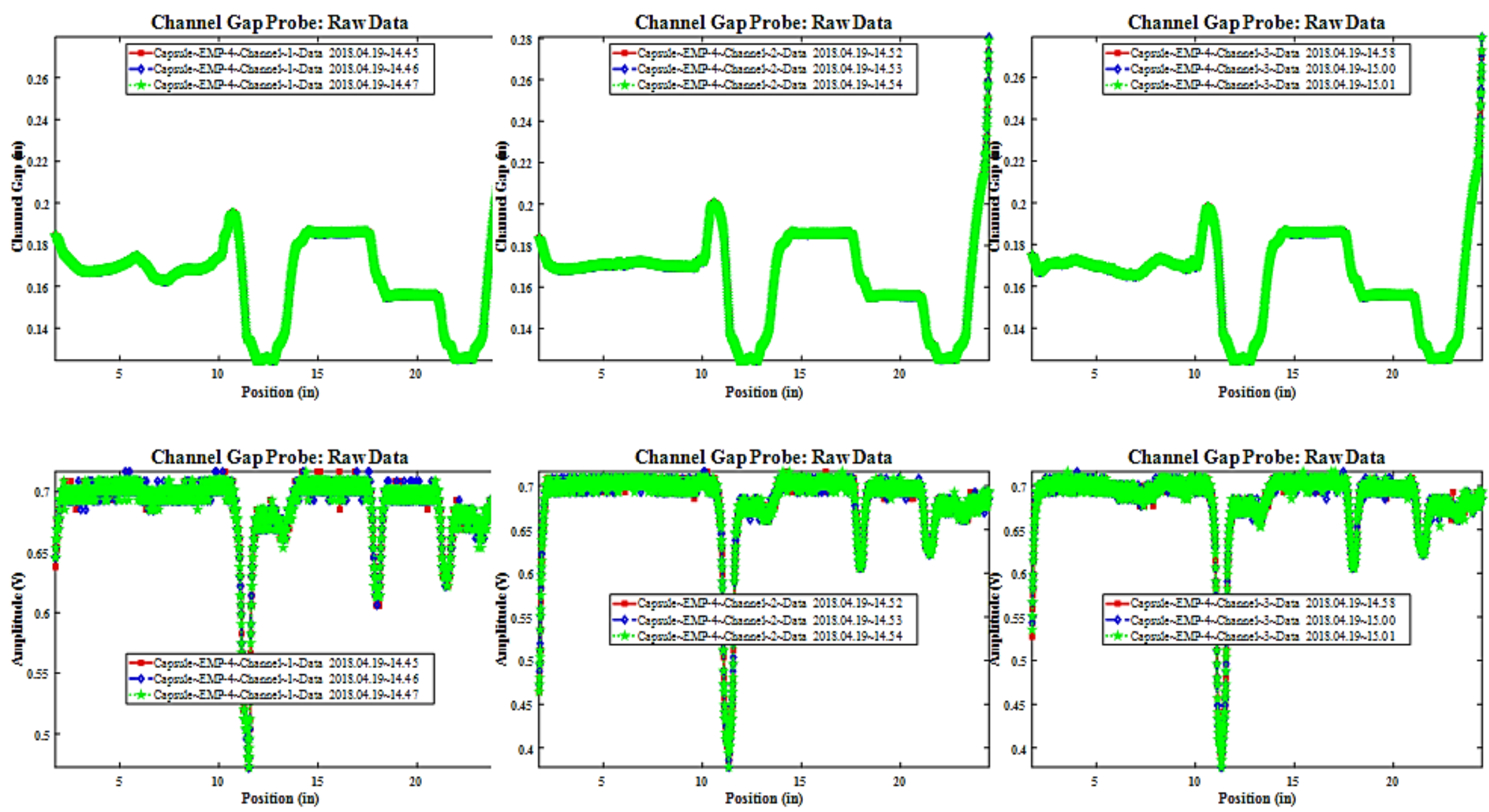

Figure 19. Variation in the Channel Gap measurements can be correlated with sharp changes in the maximum amplitude level. 


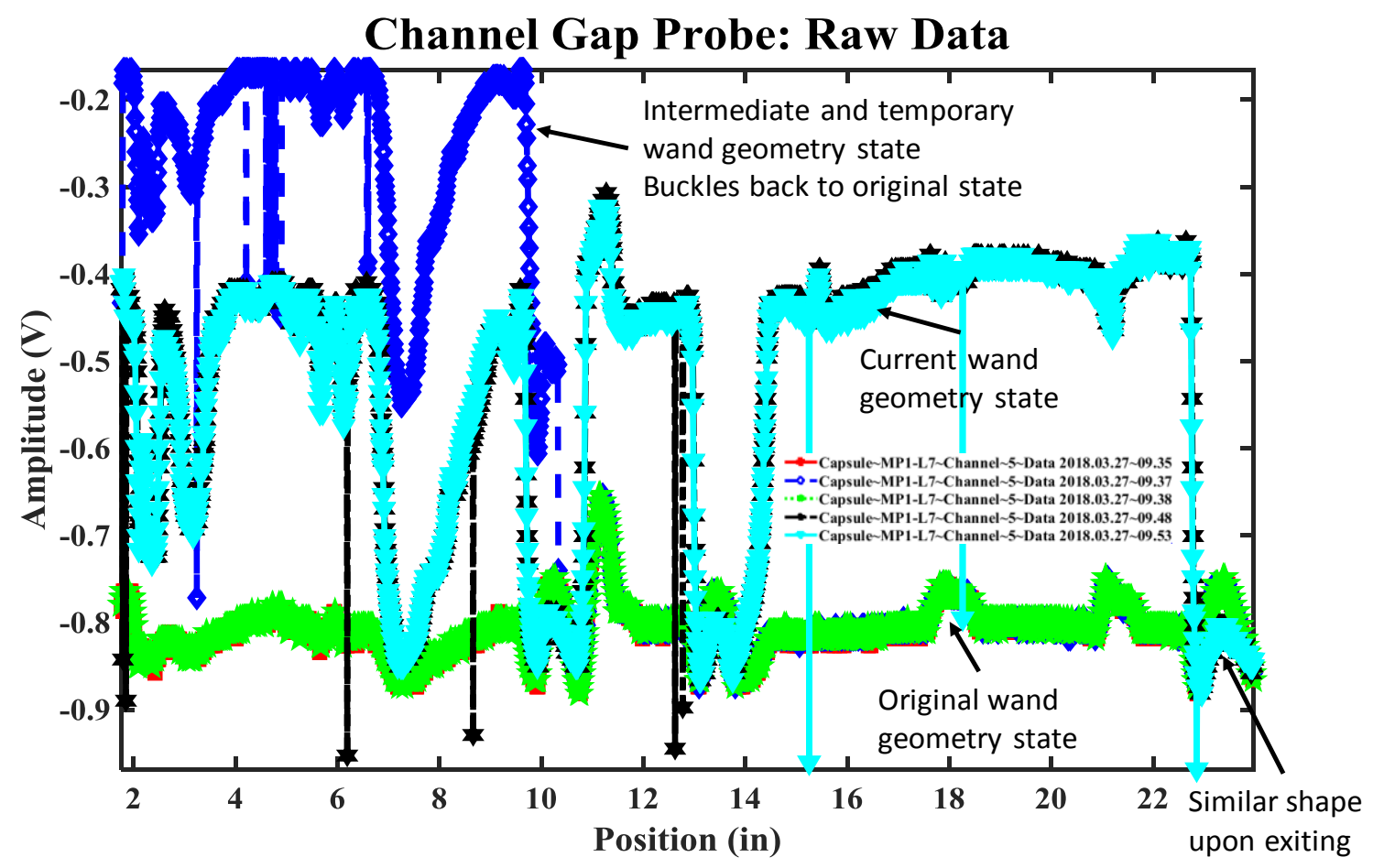

Figure 20. MP-1wand buckling can cause major changes in the patterns generated by the amplitude data.

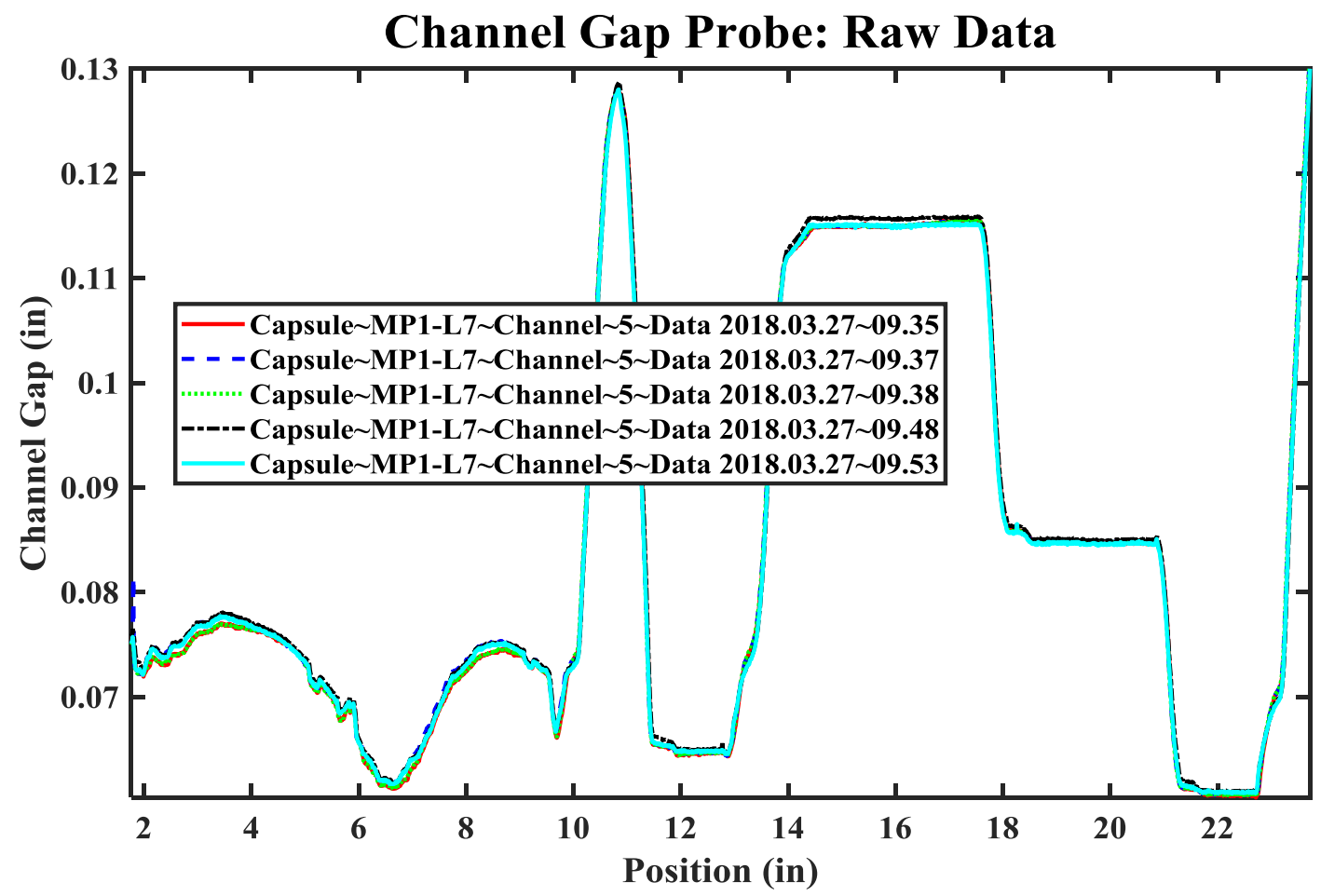

Figure 21. Despite the maximum amplitude showing major buckling in the probe, the channel width measurements are largely unaffected. The black data set has the most variation. 
Similar state changes have been seen in EMPIrE wands as shown in Figures 22 and 23. The maximum amplitude data shows signs of major buckling in the EMPIrE probe, the channel width measurements are significantly affected with a bias of $0.25 \mathrm{~mm}(0.01$ "). It is hypothesized that the bias in the EMPIrE probe is significantly larger than the MP-1 bias because the nominal channel widths are significantly larger in the EMPIrE capsules. Note that the calibration side of the plot (right side with distinctive steps) are grouped much closer. Since the calibration steps are of known height, the calibration process forces the steps to be "right" which in turn biases the capsule measurements. This is always a potential issue when making quantitative measurements based on calibration standards. Thus buckling can cause the calibration process to bias the capsule measurements while PASSING the calibration check. Only by monitoring the amplitude data can buckling and any possible biasing be detected. This is a major reason why standard capsules are used and measured at the start of the day and the end of the day. The standard capsules will detect bias in the measurements caused by a wand's baseline shift due to buckling or plastic deformation (permanent geometry change) in the wand. The amplitude data is an excellent detector of changes in the wand's geometry but not an accurate estimator of channel gap width, hence a data confidence indicator.

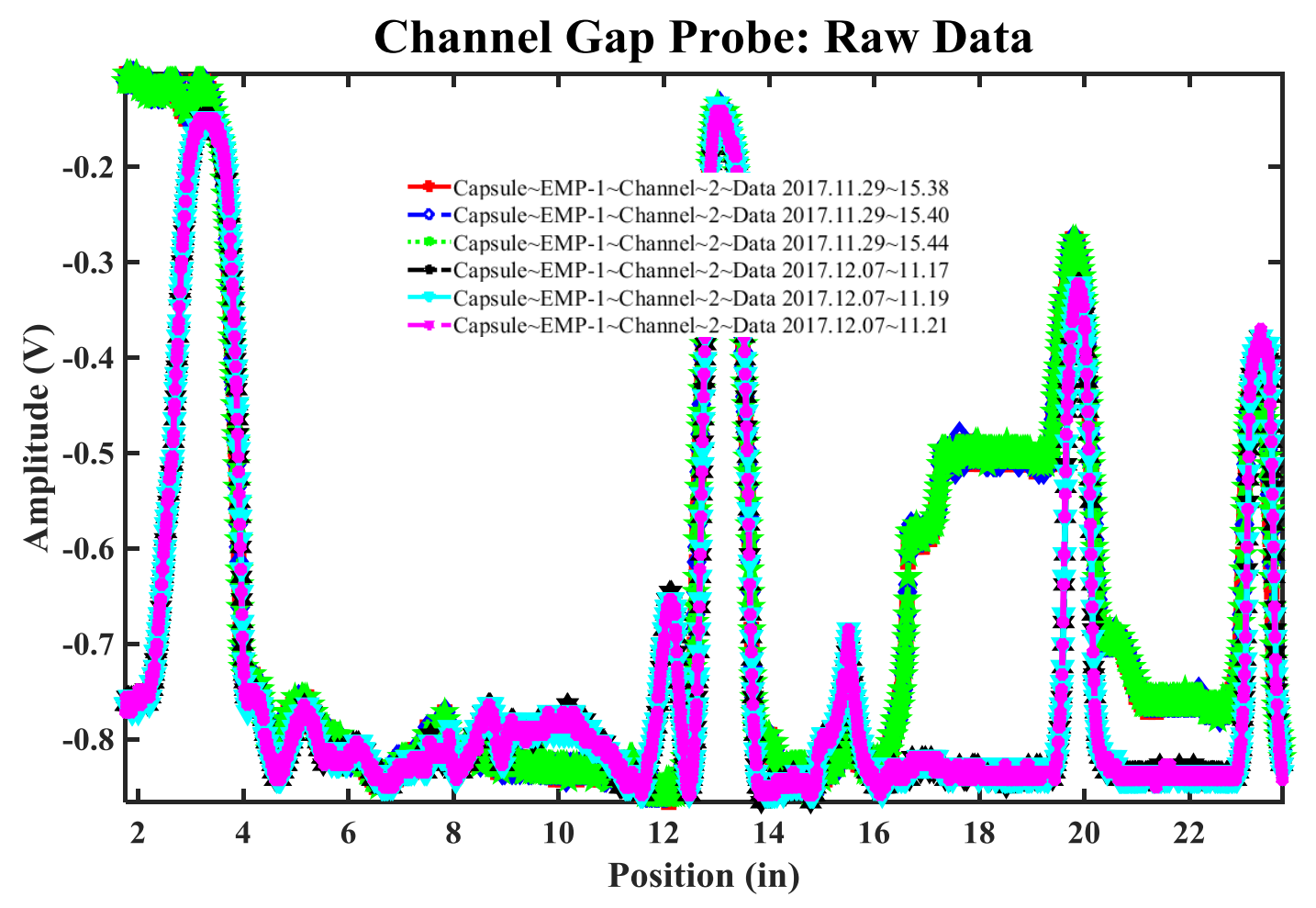

Figure 22. Empire wand buckling can cause major changes in the patterns generated by the amplitude data. 


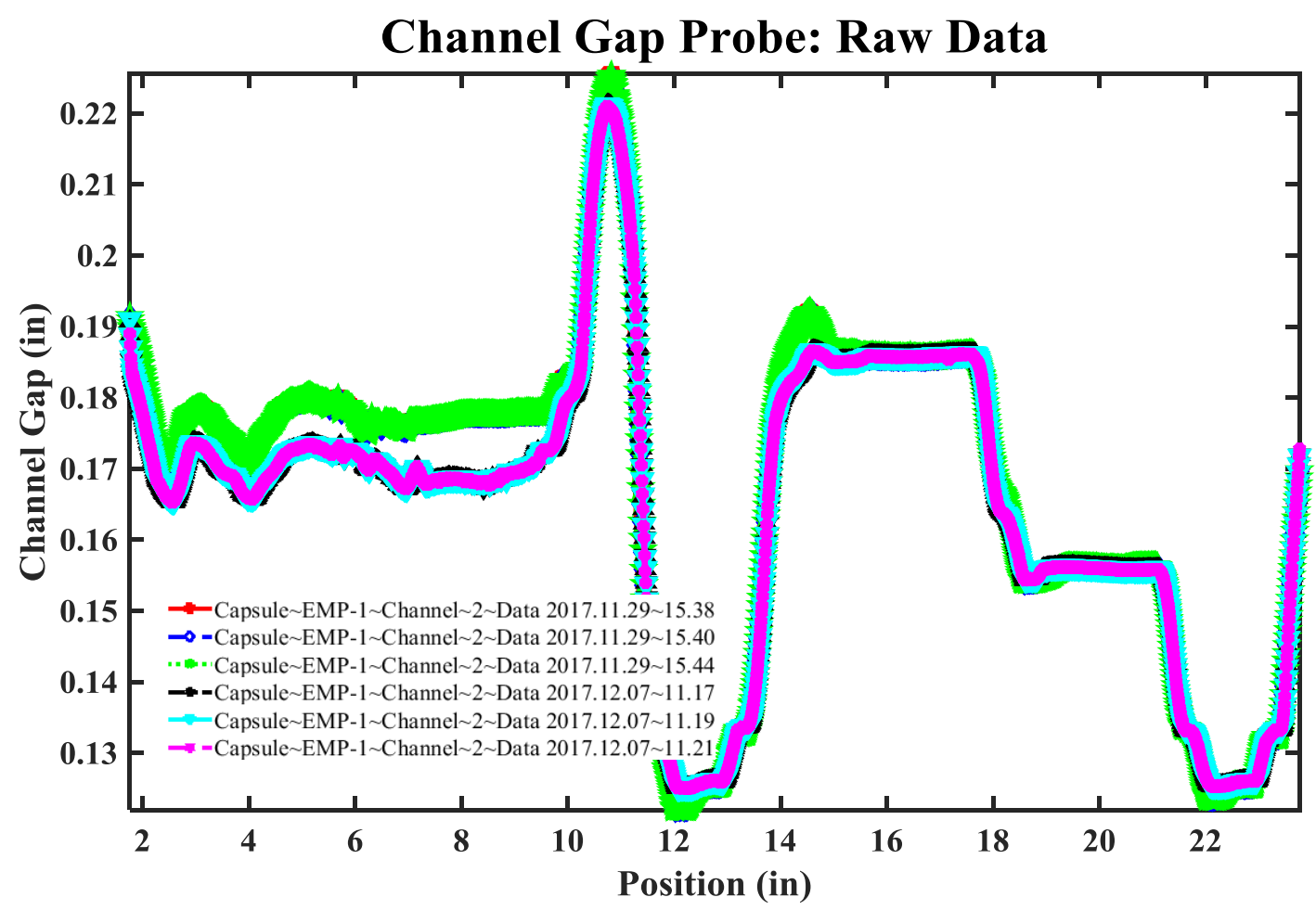

Figure 23. The maximum amplitude data shows signs of major buckling in the EMPIrE probe, the channel width measurements are significantly affected with a bias of $0.25 \mathrm{~mm}(0.01$ "). Note that the calibration check side of the plot (right side with distinctive steps) are grouped much closer. Since the calibration steps are of known height, the calibration process forces the steps to be "right" which in turn biases the capsule measurements.

\subsection{Standard Capsule}

As mentioned in the earlier section, the standard capsule is used to detect any baseline shift or biases in the CGP measurements. The capsules are geometric equivalents to the fuel capsules but have "blank" aluminum plates in the fuel slots. The aluminum plates are swaged into the channel groves to keep the plates from moving. This helps to keep the measurement deviations low from surface topography and geometric deviations.

During the EMPIrE irradiated capsule campaign, the standard capsule (affectionately labeled Dummy) was tested over a time period of 11 days. The resulting single shot plots are shown in Figure 24. There does not appear to be any evidence of baseline shifts or buckling as the data generally overlays upon itself. The data does however show a change in position registration. The black plot is slightly shifted horizontally from the rest of the data. The CGP system position encoders have a curious defect in that the relative encoder loses track of its position over time. If the operator is not diligent in resetting the location during a series of tests, the graphs can become miss-registered as demonstrated in Figure 24. Figure 25 shows the Standard deviation of the four data sets. Notice that the variance at the transition points is significantly higher which reinforces the use of the amplitude data as an indication of data confidence. Where the amplitude and channel gap measurements are stable the gap variance is nominally $0.0127 \mathrm{~mm}$ $(0.0005$ "). This variance value is significantly lower than the reproducibility values given in the next section from fueled plates even though the standard capsule measurements were made in the ATR canal. 


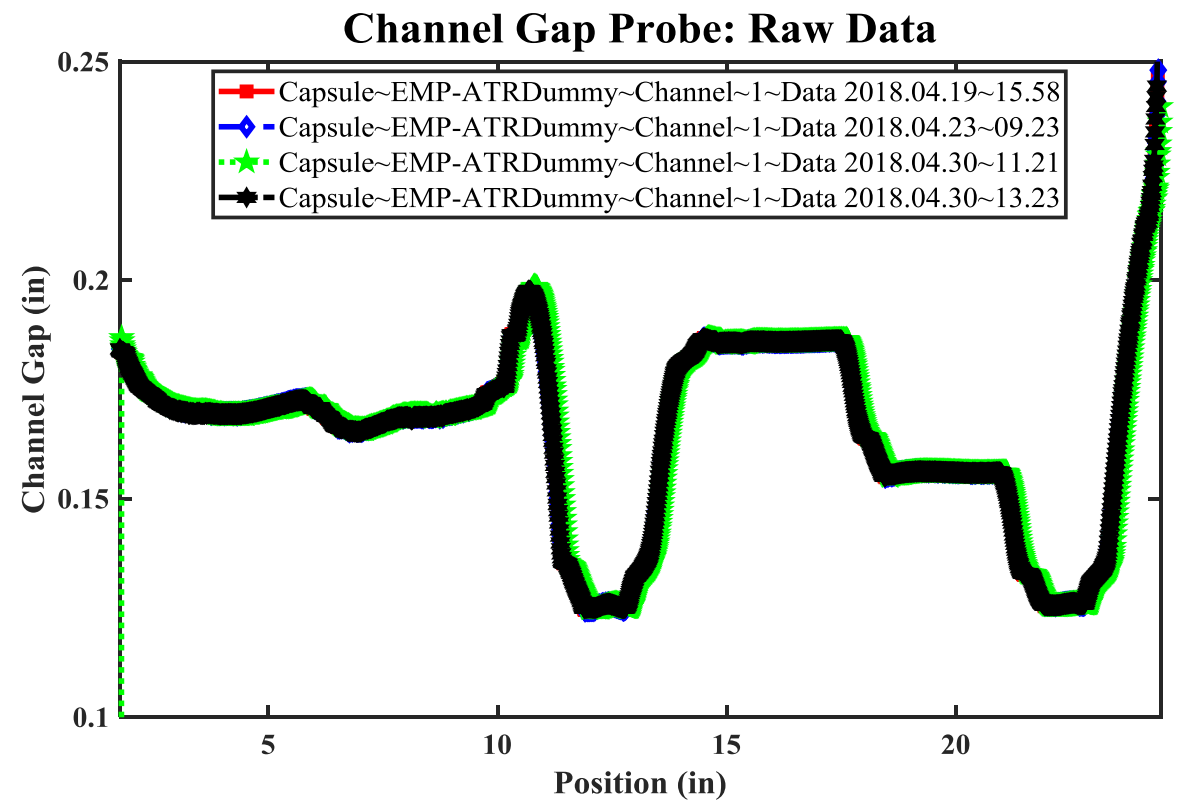

Figure 24. The CGP system can experience slight miss-registration of the wand position as indicated by the green plot.

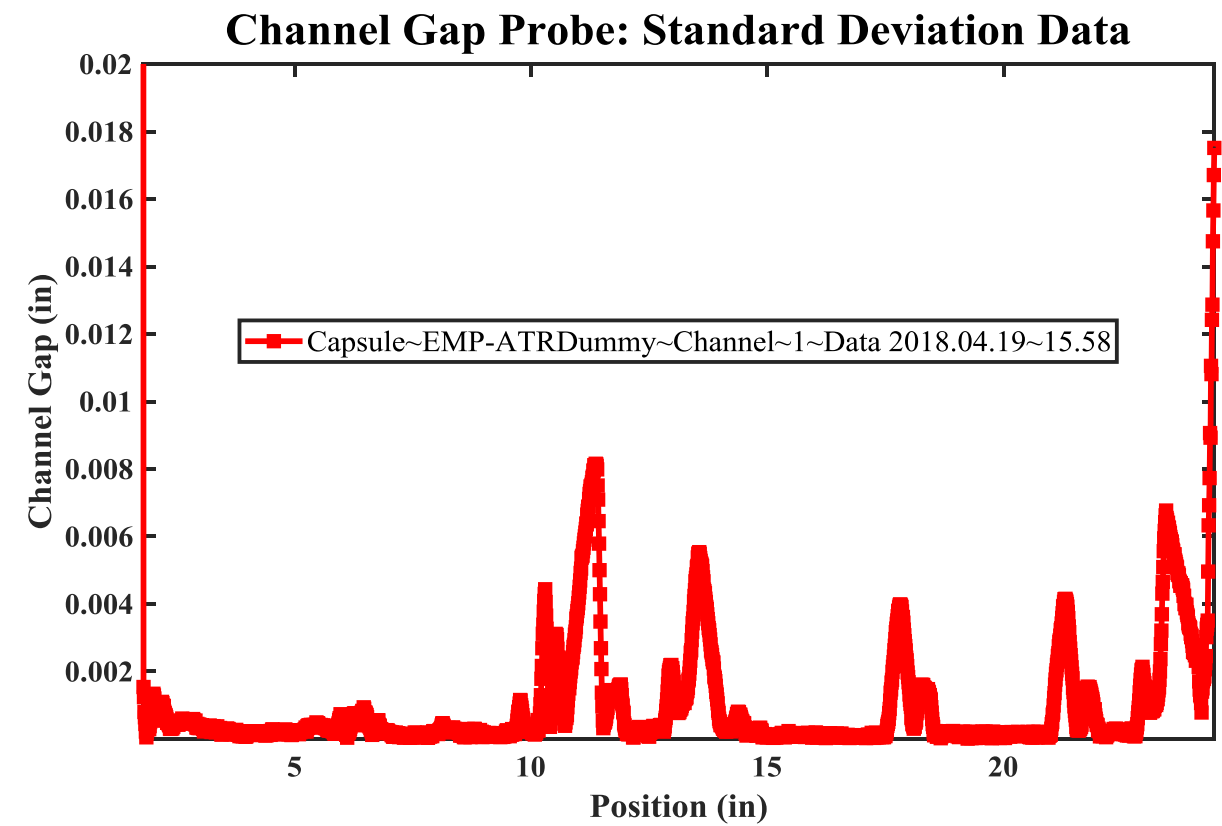

Figure 25. The standard capsule is a good indication of the best measurement resolution obtainable by the CGP. The dummy fuel plates appear to have less surface roughness and the dummy plates are rigidly fixed which combine to significantly reduce plate variability.

As stated previously, the maximum amplitude of the signal peak being followed alerts the CGP operator that the wand geometry is changing. This is demonstrated in Figure 26 by the signal dropouts in the transition regions. The data variance shown in Figure 27, confirms that the wand's measured amplitude varies significantly in the transition regions. The features noted in the amplitude plots are used to indicate any baseline shifts in the measurement system and provide an indication of the data confidence. 


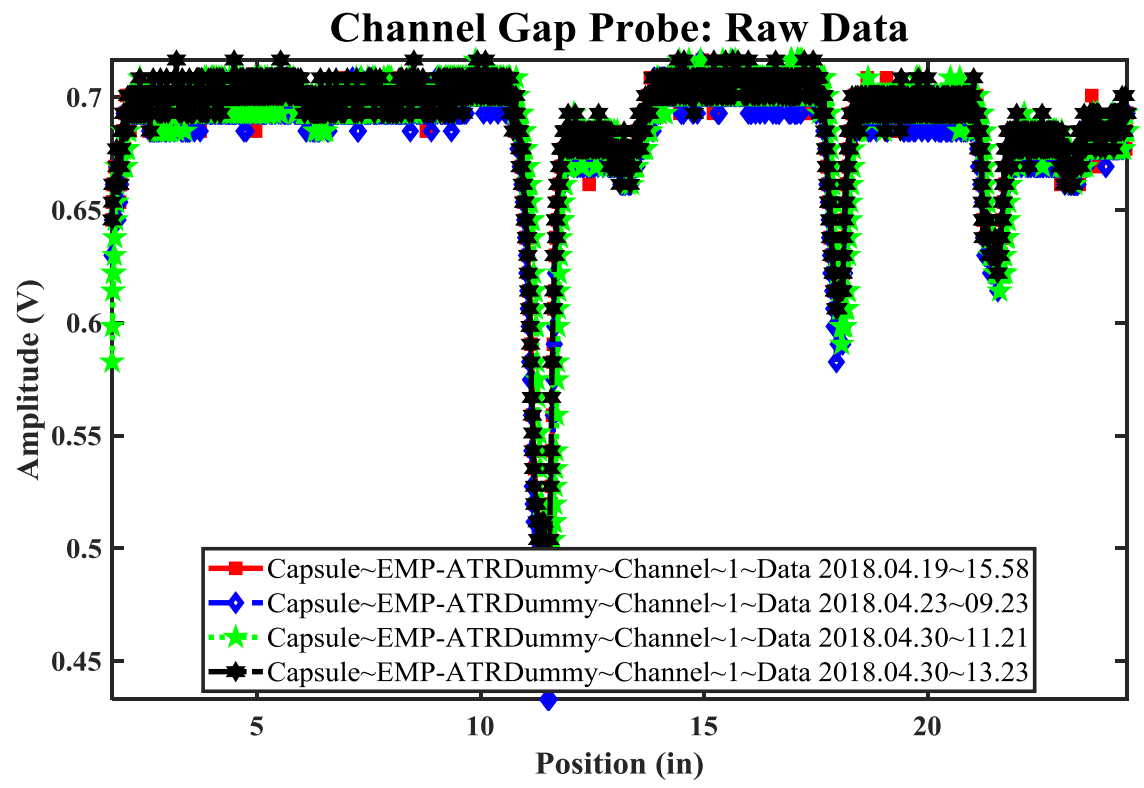

Figure 26. The signal amplitude varies significantly when the wand transitions into/out of the capsule and at the standard gap transitions in the calibration block.

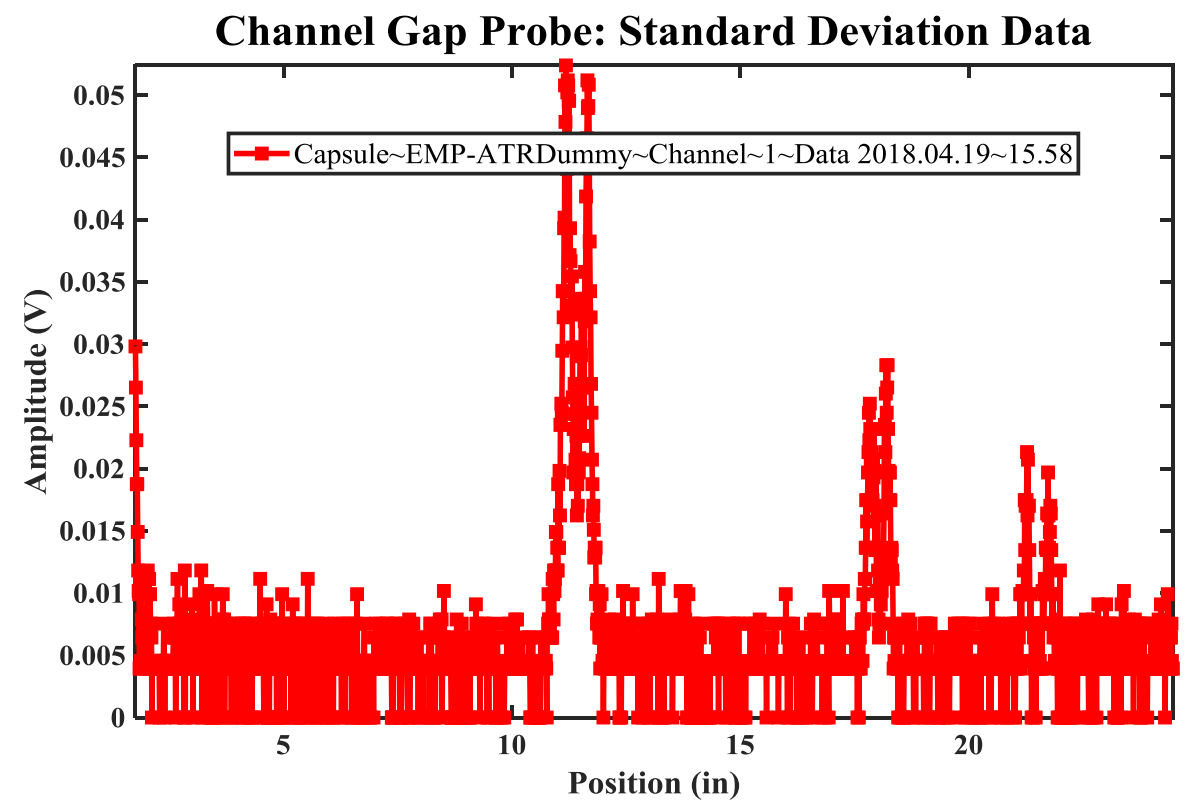

Figure 27. The signal amplitude also varies the most at the major transition locations.

\section{Repeatability \& Reproducibility}

The CGP probe is used to determine the gap distance between fuel plates for the EMPIrE and MP-1 experiments as part of the M3 fuel development program. The CGP wand is inserted between plates in the experimental fuel capsule. The M3 Fuel Development program needs this data to understand fuel plate swelling and plate deformation. The program would like to be alerted when the measured deformation is abnormal/unexpected by comparison to modeling data. The threshold where signal is separated from the noise is based on the repeatability of the data. 
The CGP works by measuring the time of flight of the ultrasonic signal between two nominally parallel ultrasonic transducer plates. The goal of this section is to provide an estimate of the repeatability and reproducibility of the CGP measurement from a fresh fuel capsule, EMPIrE 8. These statistical values are needed to understand the minimum resolution of the CGP measurements and determine the validity of the measured geometric variations.

- Repeatability is defined as taking replicate measurements without disturbing the set-up and under identical conditions.

- Reproducibility is defined as taking replicate measurements after the set-up was disturbed and put back into a similar state and perhaps under different conditions.

- The repeatability value will indicate significant changes between the replicate data. Reproducibility value will indicate significant changes between replicate data sets (baseline drift).

- Repeatability and reproducibility values are the thresholds to let the experimenter know that significant changes have occurred outside of typical measurement variations.

The statics for the ultrasonic transducer amplitude data are also an important diagnostic measure. The CGP measurement is sensitive to the geometry of the measurement probe. The changes in UT amplitude for specific gap distances indicate geometry changes in the probe. Probe geometry changes are known to affect the measurements. The amplitude information is an opportunity to estimate the validity of the gap measurements. For further details on how the repeatability and reproducibility estimates have been obtained and limitations see TEV-3363[8].

The EMPIrE 8 capsule has been chosen to perform the baseline statistical measurements. It is presumed that the EMPIrE 8 capsule is representative of the rest of the capsules and contains most of the "features" found in the other capsules. Since the sensing elements on the channel gap wand are $12.7 \mathrm{~mm}(0.5$ ") long, any feature such as edge curling due to the shearing process will affect the measurement for $12.7 \mathrm{~mm}$ (0.5”). Figure 28 shows the gap displacement profile from channel 2. The plate edges are at 58.4 (2.3), 160 (6.3) and 261.6 (10.3) $\mathrm{mm}$ (inches). The valid data collection ranges are from 71.1 (2.8) to 147.3 (5.8) and 172.7 (6.8) to 248.9 (9.8) $\mathrm{mm}$ (inches) to reduce the variations caused by edge deformation.

Channel Gap Probe: Raw Data

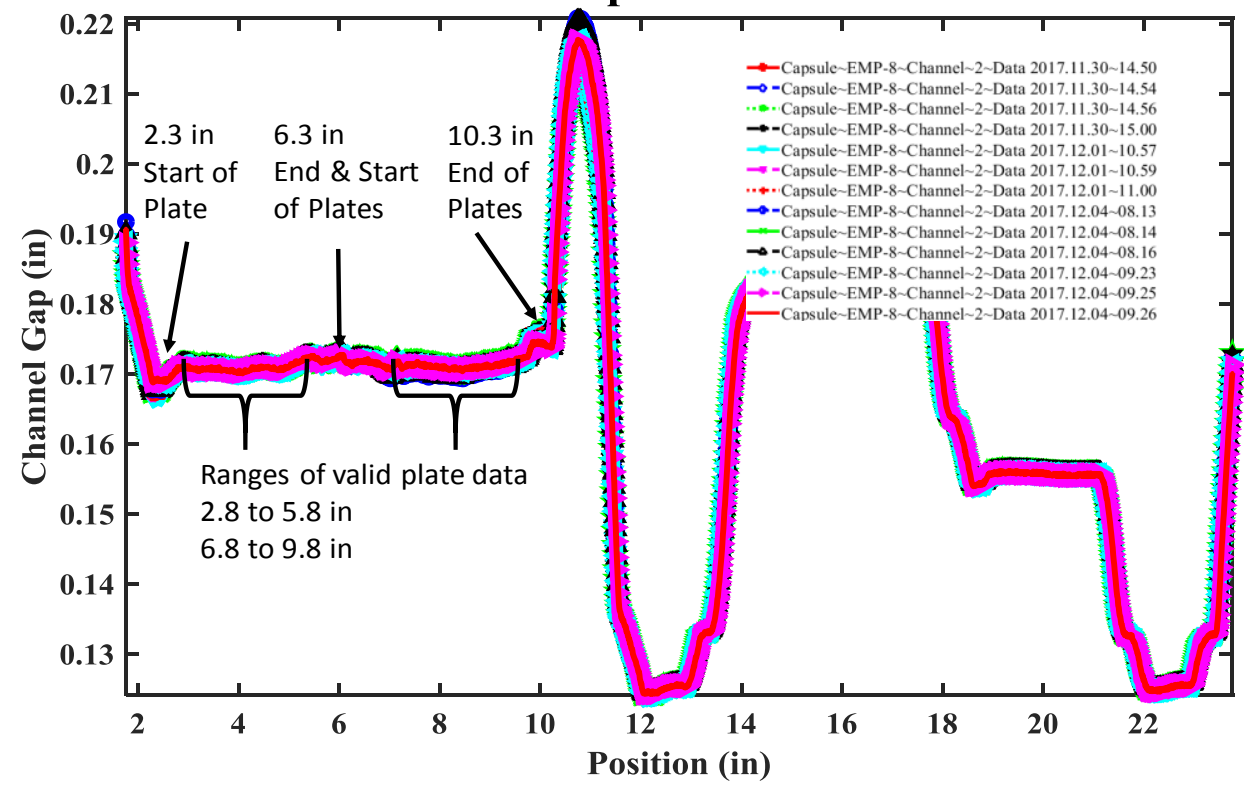

Figure 28. Example graph showing the raw CGP data for channel 2 in the EMPIrE 8 capsule. The plot also shows the valid locations to estimate the measurement statistics. 
Reproducibility and Repeatability values are calculated by the method discussed in ASTM Standard E691-16 [9]. It is unfortunate to note that this standard contained a number of mistakes and the methodology is not clear. The TEV-3363 results implemented using ASTM Standard E691-16 have been collaborated by the checker in Appendix C. All of the Reproducibility and Repeatability graphs can be found on the share drive [9] in this file, CGP EMP8 Reproduce Repeat.pptx.

The variation of the resulting measurements along the plate is fuel plate dependent and random with respect to plate location. The processed data provides a plethora of statistical data along the length of the plate. The location of the high variation points will not be known in advance and it is impractical to consider variations for all the locations along the capsule thus the maximum variation in the valid data range is reported. This conservative approach is being taken by reporting the worst case statistical values for the gap and UT amplitude. The objective of this reporting strategy is not to be "right" but conservative.

It should be noted that due to a software glitch in the CGP system when outputting the amplitude data, the amplitude data locations are offset by the thermocouple distance of $44.45 \mathrm{~mm}(1.75$ ”). The gap location values are accurate. The worst case data are reported for the location ranges of 71.1 (2.8) to 147.3 (5.8) and 172.7 (6.8) to 248.9 (9.8) $\mathrm{mm}$ (inches). Based on preliminary statistics from the other capsules, EMPIrE 8 has relatively low variations.

How the Reproducibility and Repeatability data sets are created/parsed is shown in Figure 29. All of the EMPIrE 8 data can be found on the M3 share [10]. Table 1 shows the results from using the worst case Reproducibility and Repeatability values within the valid data ranges for all three channels in EMPIrE 8. The averages from the three channels were determined and then reported as the statistical results. The channel averages are interpreted as Rules Of Thumb To Alert Of Possible Indications. The single location threshold is estimated from the STD generated by the replicate data.

- EMPIrE-8 contains 4 reproducibility sets

- Capsule removed between days

- 11.30

- 12.01

- 12.04 before 09:00

- 12.04 after 9:00

- Foot was replaced

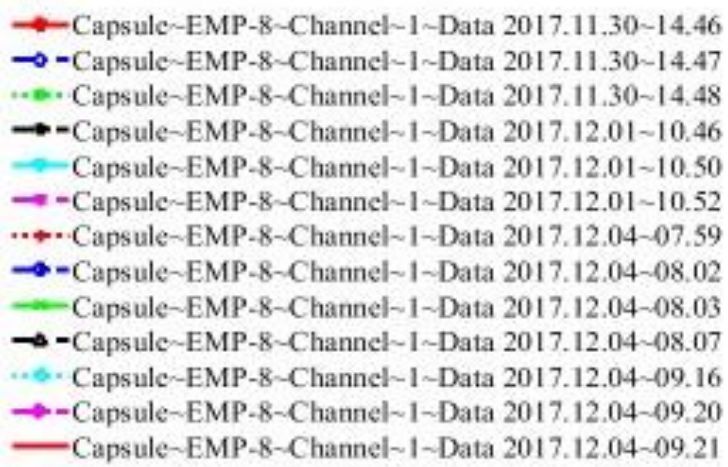

$\rightarrow$ Capsule-EMP-8-Channel-1 -Data 2017.11.30 -14.46 $\leadsto-$ Capsule-EMP-8-Channel-1 Data 2017.11.30 -14.47 *-. Capsule-EMP-8-Channel 1-Data 2017.11.30 14.48 $\rightarrow$-Capsule-EMP-8-Channel 1-Data 2017.12.01 10.46 -Capsule-EMP-8-Channel-1-Data 2017.12.01 10.50 $\rightarrow-$ Capsule-EMP-8 -Channel - 1 Data 2017.12.01 10.52 *-* Capsule-EMP-8-Channel 1-Data 2017.12.04-07.59 $\rightarrow$-Capsule-EMP-8-Channel 1 -Data 2017.12.04-08.02 -Capsule-EMP-8-Channel 1 - Data 2017.12.04-08.03 $\rightarrow$-Capsule-EMP-8 -Channel - Data 2017.12.04 -08.07 *-Capsule-EMP-8-Channel-1 -Data 2017.12.04-09.16 $\rightarrow$-Capsule-EMP-8-Channel 1-Data 2017.12.04-09.20 -Capsule-EMP-8-Channel 1 Data 2017.12.04-09.21

- Repeatability and Reproducibility calculated using ASTM E691-16 Standard: Standard Practice For Conducting an Interlaboratory Study to Determine the Precision of a Test Method

Figure 29. There were four reproducibility sets and there were at least 3 replicates in each set. The separate reproducibility sets are defined by dates and times when the data files were collected. 
Table 1 . The worst case Reproducibility and Repeatability values taken from the channel gap and UT amplitude profiles.

\begin{tabular}{|l|r|r|l|l|l|l|l|}
\hline & \multicolumn{4}{|c|}{ Channel Gap } & \multicolumn{3}{c|}{ UT Amplitude } \\
\hline Capsule & Channel \# & STD & Reproducibility & Repeatability & STD & Reproducibility & Repeatability \\
\hline & & (0.001 in) & (0.001 in) & (0.001 in) & (V) & (V) & (V) \\
\hline \hline Empi-8 & 1 & 1 & 1 & 0.3 & 0.035 & 0.04 & 0.006 \\
\hline & 2 & 0.7 & 0.8 & 0.2 & 0.035 & 0.04 & 0.014 \\
\hline & 3 & 0.8 & 0.9 & 0.2 & 0.04 & 0.045 & 0.013 \\
\hline
\end{tabular}

- The data observations were made only in the well behaved portions (sweet spot) of the fuel plates.

- Observations made along the CGP wand draw length

- $12.7 \mathrm{~mm}(0.5$ ") in from each plate edge along the length

- Expected gap repeatability and reproducibility between ATR runs

- Conservative gap width repeatability $0.0076 \mathrm{~mm}(0.0003$ ")

- Conservative gap width reproducibility $0.023 \mathrm{~mm}(0.0009 ")$

- Three run gap width STD $0.021 \mathrm{~mm}(0.00083$ ”)

- Expected Amplitude repeatability and reproducibility between ATR runs

- Conservative amplitude repeatability $0.014 \mathrm{~V}$

- Conservative amplitude reproducibility $0.045 \mathrm{~V}$

- Three run amplitude STD $0.04 \mathrm{~V}$

\section{REPRESENTATIVE CHANNEL GAP PROBE DATA}

Graphs from representative capsules, EMPIrE-2 and EMPIrE-7 will be shown below. The reader should be able to see the trends developed in the three different channels, 1, 2, 3. The outside channels show more curvature than the middle channel. The amplitude data for all three channels shows consistent nominal pattern. If there is a significant change in the amplitude data, the resulting channel gap data should be scrutinized more thoroughly. All of the EMPIrE data can be found in the M3 share directory [10] or in the M3 records archives.
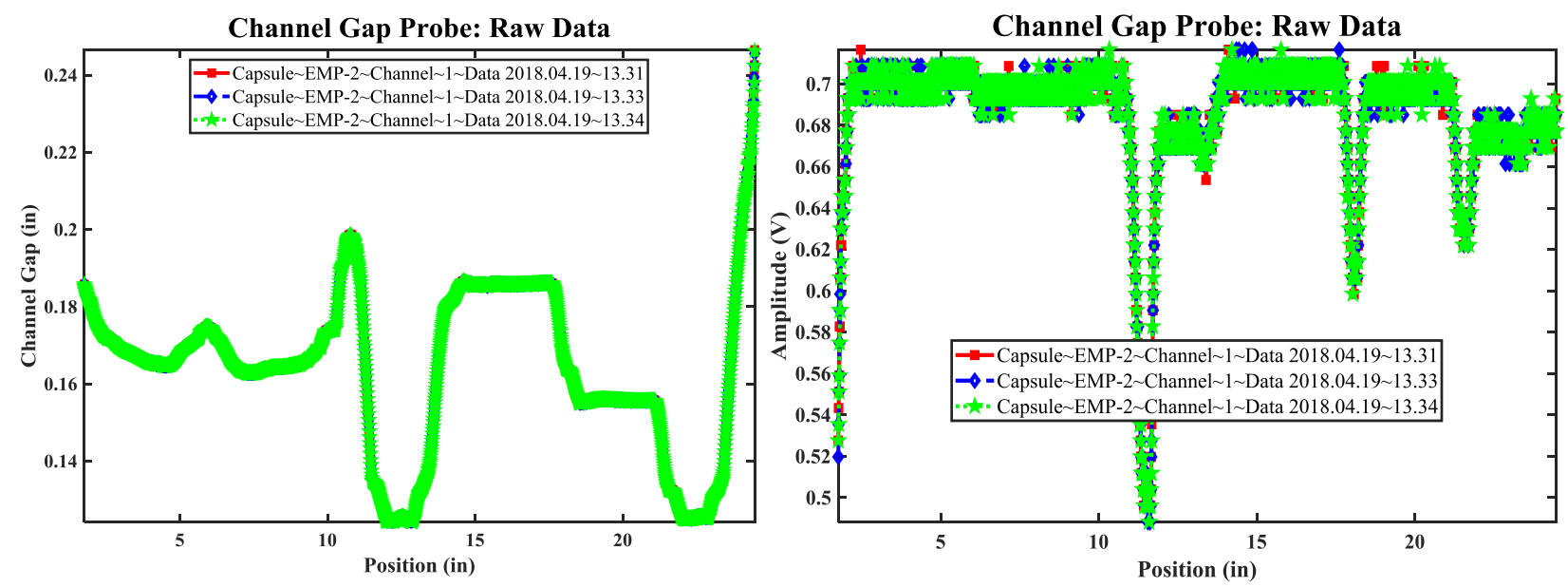

Figure 30. The channel gap width (l) and amplitude (r) data for channel 1 within EMPIrE capsule 2. 
Channel Gap Probe: Raw Data

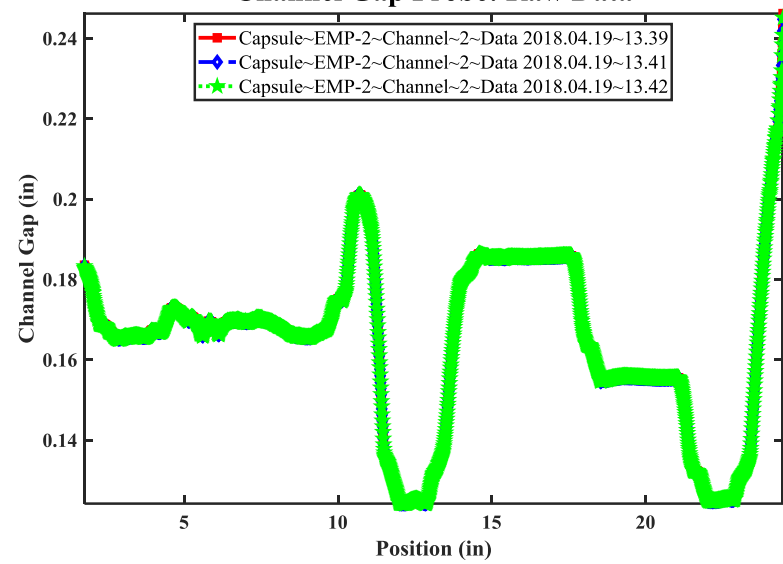

Channel Gap Probe: Raw Data

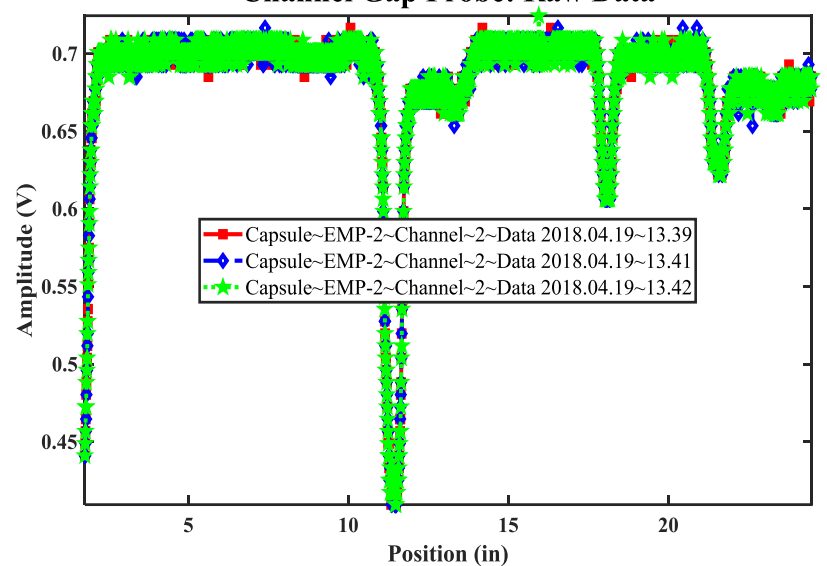

Figure 31. The channel gap width (l) and amplitude (r) data for channel 2 within EMPIrE capsule 2.
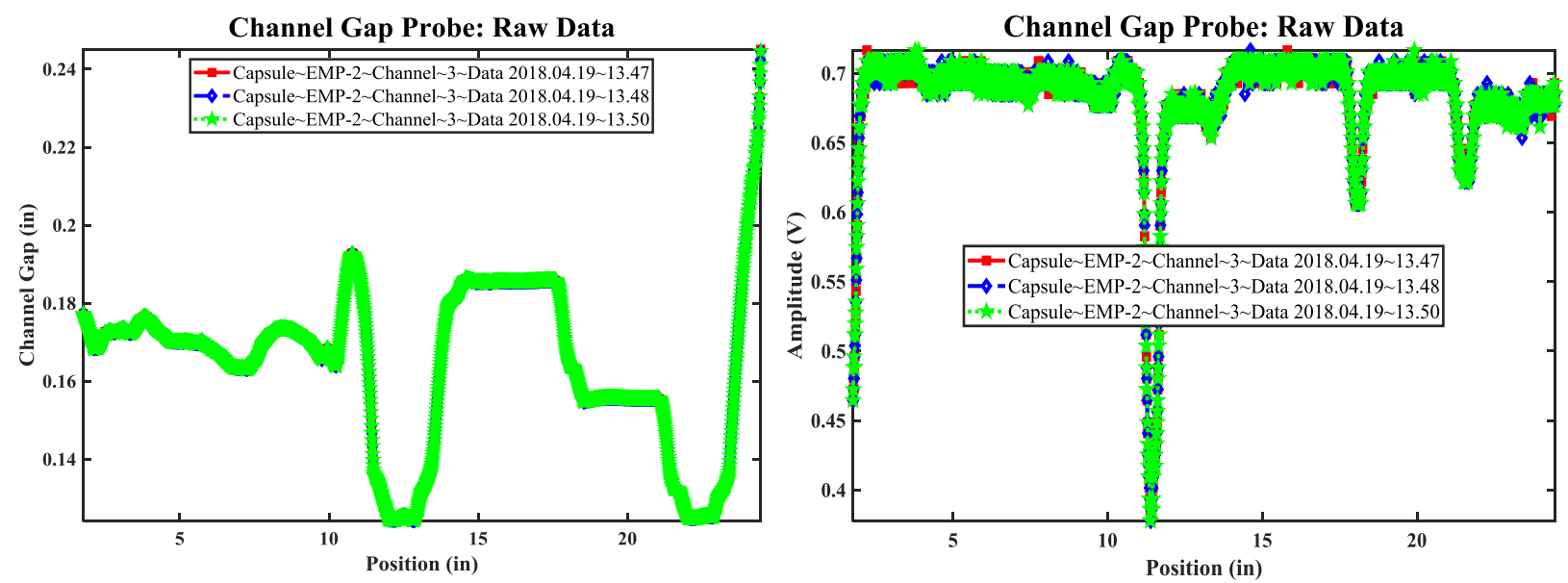

Figure 32. The channel gap width (l) and amplitude (r) data for channel 3 within EMPIrE capsule 2.
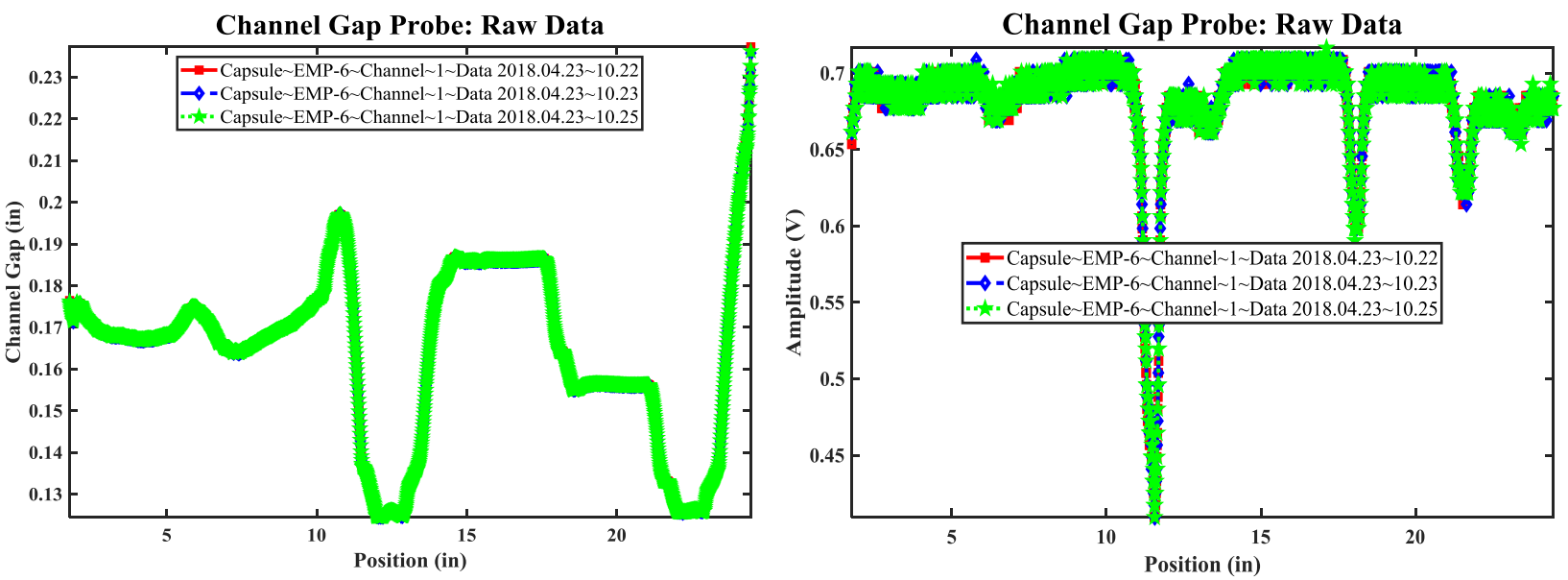

Figure 33. The channel gap width (1) and amplitude (r) data for channel 1 within EMPIrE capsule 6. 

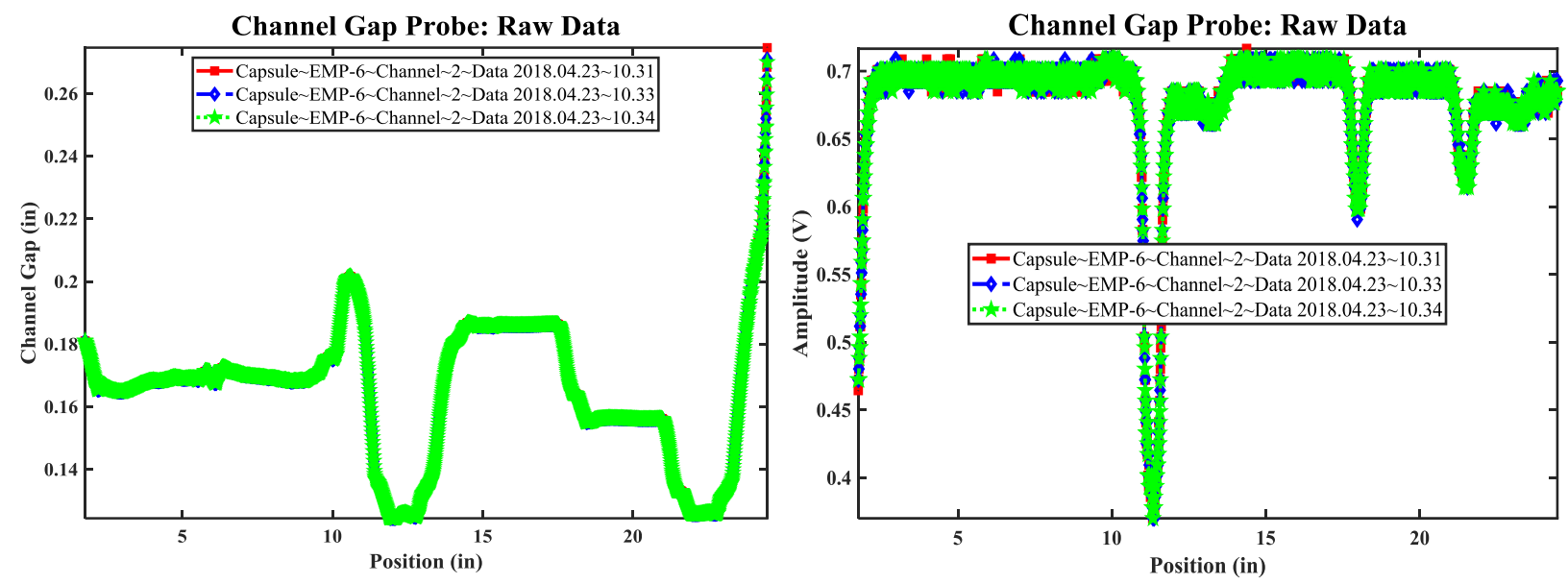

Figure 34. The channel gap width (l) and amplitude (r) data for channel 2 within EMPIrE capsule 6.
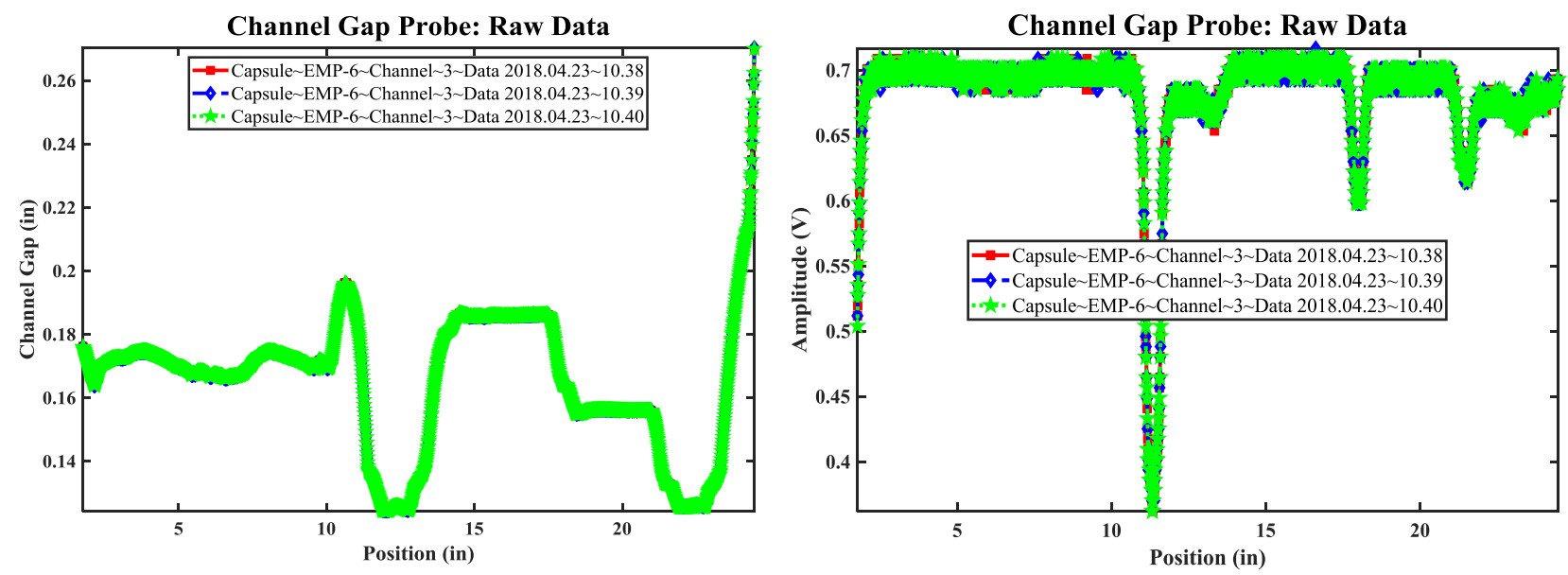

Figure 35. The channel gap width (l) and amplitude (r) data for channel 3 within EMPIrE capsule 6.

\section{Channel Gap Probe Data}

This section presents the CGP data collected on 8 EMPIrE capsules (EMP-1 through 8) before they were inserted into ATR (referred as the Baseline data), as well as the CGP data collected on those capsules after they were irradiated during ATR cycle 162B (referred to Cycle 1 data). The change in the channel gap widths (Cycle 1 data - Baseline data) for EMP-1 through 8 is also presented and discussed. For capsules EMP-9 through 12, only Baseline data has been collected since they were not irradiated in cycle 162B. Therefore this data is in Appendix B.

\subsection{Baseline and Cycle 1 CGP Data}

Figure 36 through Figure 43 show Baseline CGP data overlaid with the CGP data collected after Cycle 1 (ATR cycle 162B). Each figure contains three plots corresponding to the three channels in the EMPIrE capsules. Between the three plots are colored labels that indicate the type of plates that surround each channel. The labeling and colors originated from the Execution Plan for EMPIrE and an explanation of the plate type indicators are summarized in Table 2 [11]. Each channel gap plot contains the channel gap swipes for the Baseline and Cycle 1 swipes in light blue and red, respectively. The average of those swipes are in dark blue and red. The blue and orange shaded regions indicate where the plates begin and 
end. The direction of the coolant flow during irradiation is from right to left in each figure, which is also indicated by the blue arrows on the right side of each channel plot.

Table 2. Key for the plate type indicators in CGP data figures.

\begin{tabular}{|l|l|}
\hline \multicolumn{1}{|c|}{ Plate Type Indicators } & \multicolumn{1}{c|}{ Explanation } \\
\hline ZrN & Zirconium Nitride coating \\
PVD & Zirconium Nitride/Aluminum Nitride double layer coating \\
ALD & Physical Vapor Deposition method of coating application \\
NO HEAT & Atomic Layer Deposition method of coating application \\
HEAT & No heat treatment of fuel particles \\
STD & With heat treatment of fuel particles \\
MOD & Standard particle size distribution \\
ZERO & Modified particle size distribution \\
MONOLITHIC PVD & Powder without fine particles \\
MONOLITHIC CO-ROLLED & Monolithic fuel with Zr barrier application by PVD method \\
U10Mo & Monolithic fuel with Zr barrier application by co-rolling method \\
CERCA POWDER & U-10wt\% Mo fuel powder \\
\hline
\end{tabular}

Figure 36 shows the CGP data for EMP-1. The data from the Baseline and Cycle 1 swipes generally follow each other through each of the channels with the exception being at the exit and entrance of channel 2 ( 0 and 8 inches from the bottom plate's trailing edge). As mentioned in Section 4, this is likely due to variations at the edges of the plates that gets propagated for 0.5 " since the sensing elements on the CGP wand are 0.5 " long. Therefore the valid data collection ranges are from 0.5 to 3.5 inches and 4.5 to 7.5 inches from the bottom plate's trailing edge in all of Section 6's plots.

Around 3.7 inches from the bottom plate's trailing edge the CGP data in EMP-1 channel 3 dropped out in one of the Baseline swipes causing the data to go off scale. Data dropouts also occurred in channel 2 of EMP-2 (Figure 37) at about 4.4 inches from the trailing edge and in channel 3 of EMP-7 (Figure 42) from about 1.25 to 2 inches from the trailing edge. In general, all 8 capsules show the Cycle 1 data following the Baseline data with offsets nominally less than 0.004 inches. The next section shows the difference between the Baseline and Cycle 1 data sets.

To assure the channel gap probe retained its ability to provide repeatable data a dummy capsule with swaged dummy plates was fabricated for use at the ATR canal. Periodically during CGP measurements at the ATR canal, measurements are taken on the dummy capsule to assure that the CGP system has not changed. Figure 44 shows the first set of CGP data taken on the dummy capsule on April 19, 2018 (blue traces) and the last set of data taken on April 30, 2018 (red traces). Both data sets are nearly on top of each other except for small variations near the edges of the plates. The average of both data sets was calculated and the difference between them is presented in the next section. 

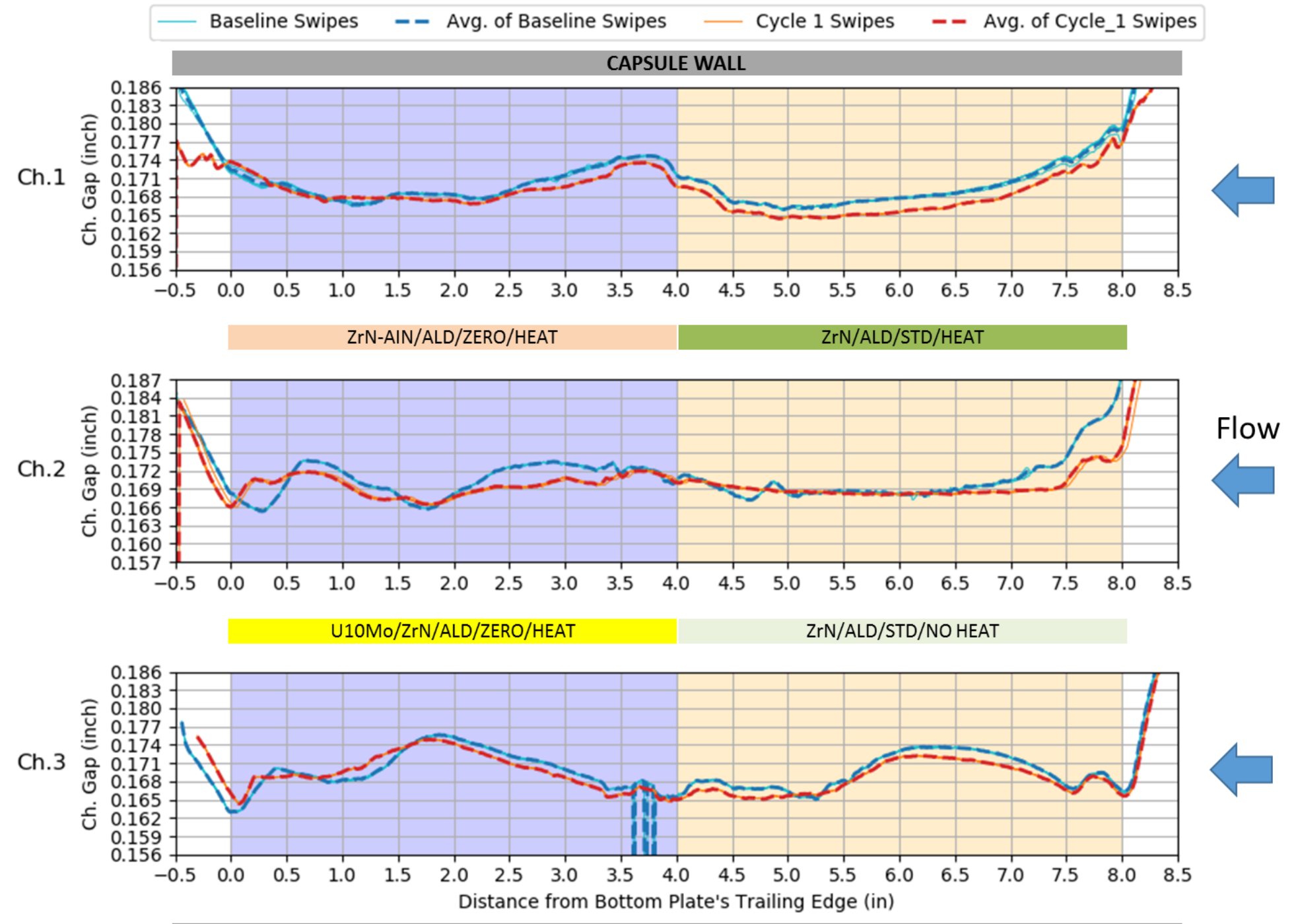

CAPSULE WALL

Figure 36. Baseline (blue traces) and Cycle 1 (red traces) CGP measurements for capsule EMP-1 with descriptions of the plate types between the plots of channel gap width. 

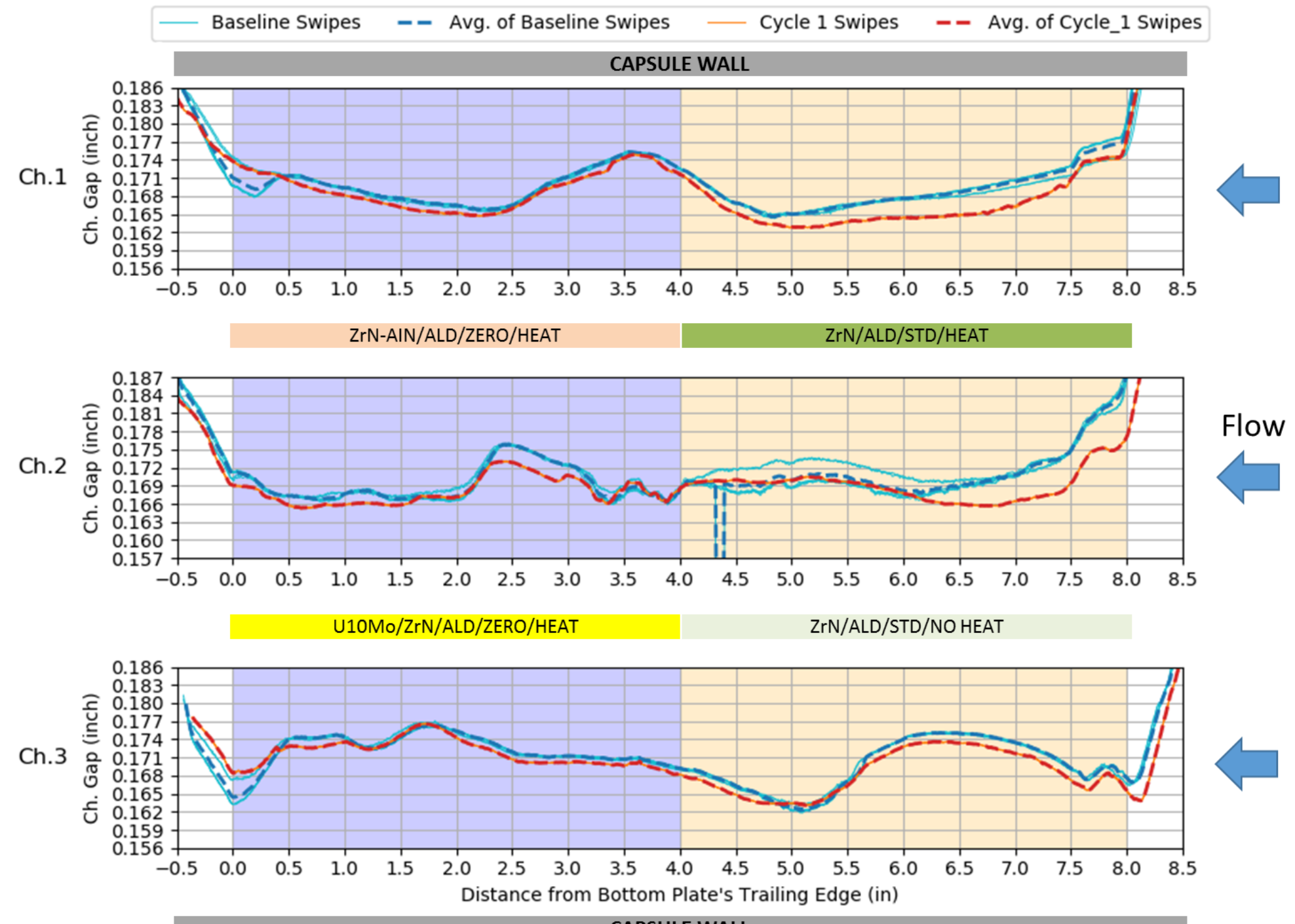

CAPSULE WALL

Figure 37. Baseline (blue traces) and Cycle 1 (red traces) CGP measurements for capsule EMP-2 with descriptions of the plate types between the plots of channel gap width. 

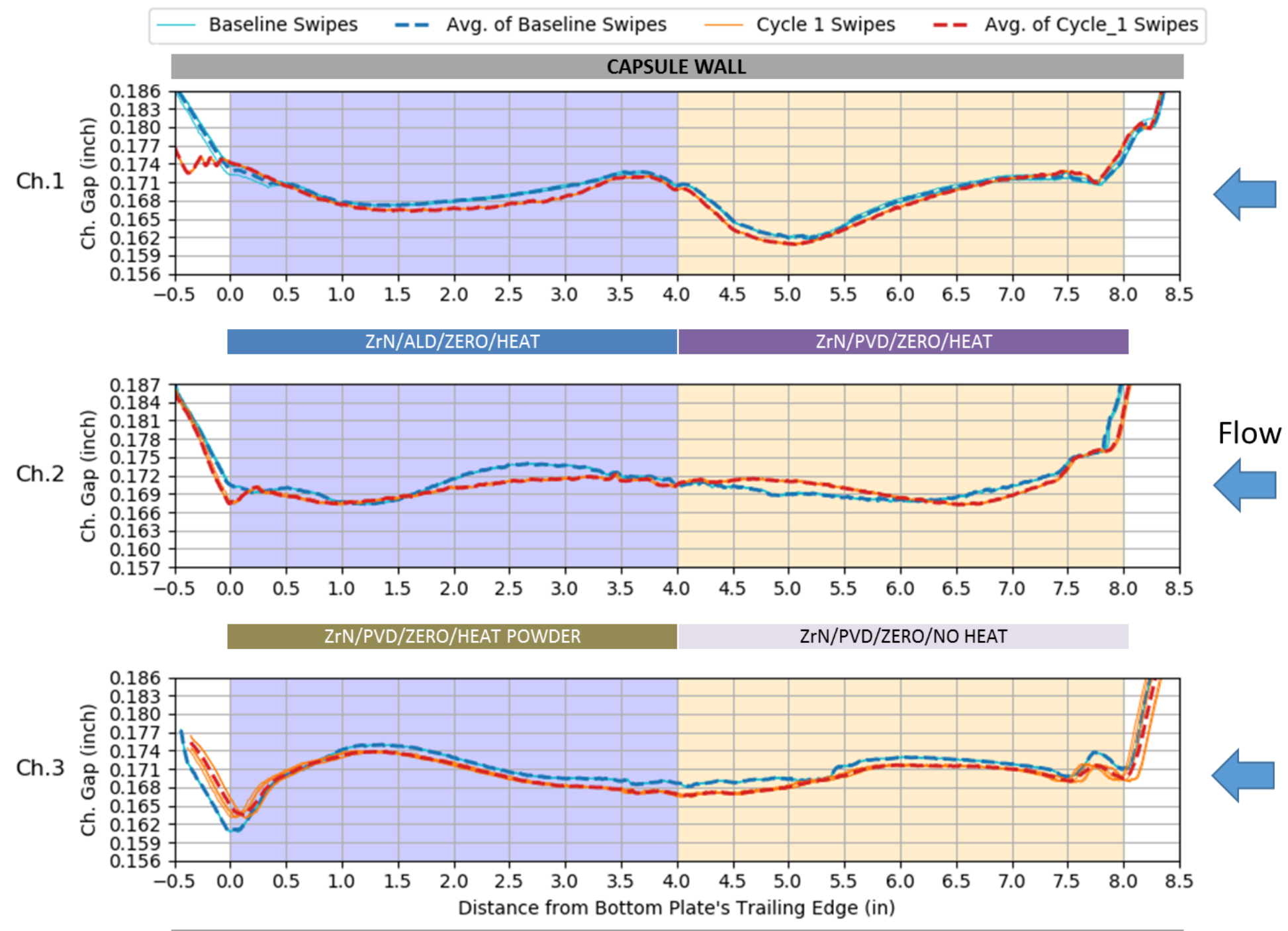

CAPSULE WALL

Figure 38. Baseline (blue traces) and Cycle 1 (red traces) CGP measurements for capsule EMP-3 with descriptions of the plate types between the plots of channel gap width. 


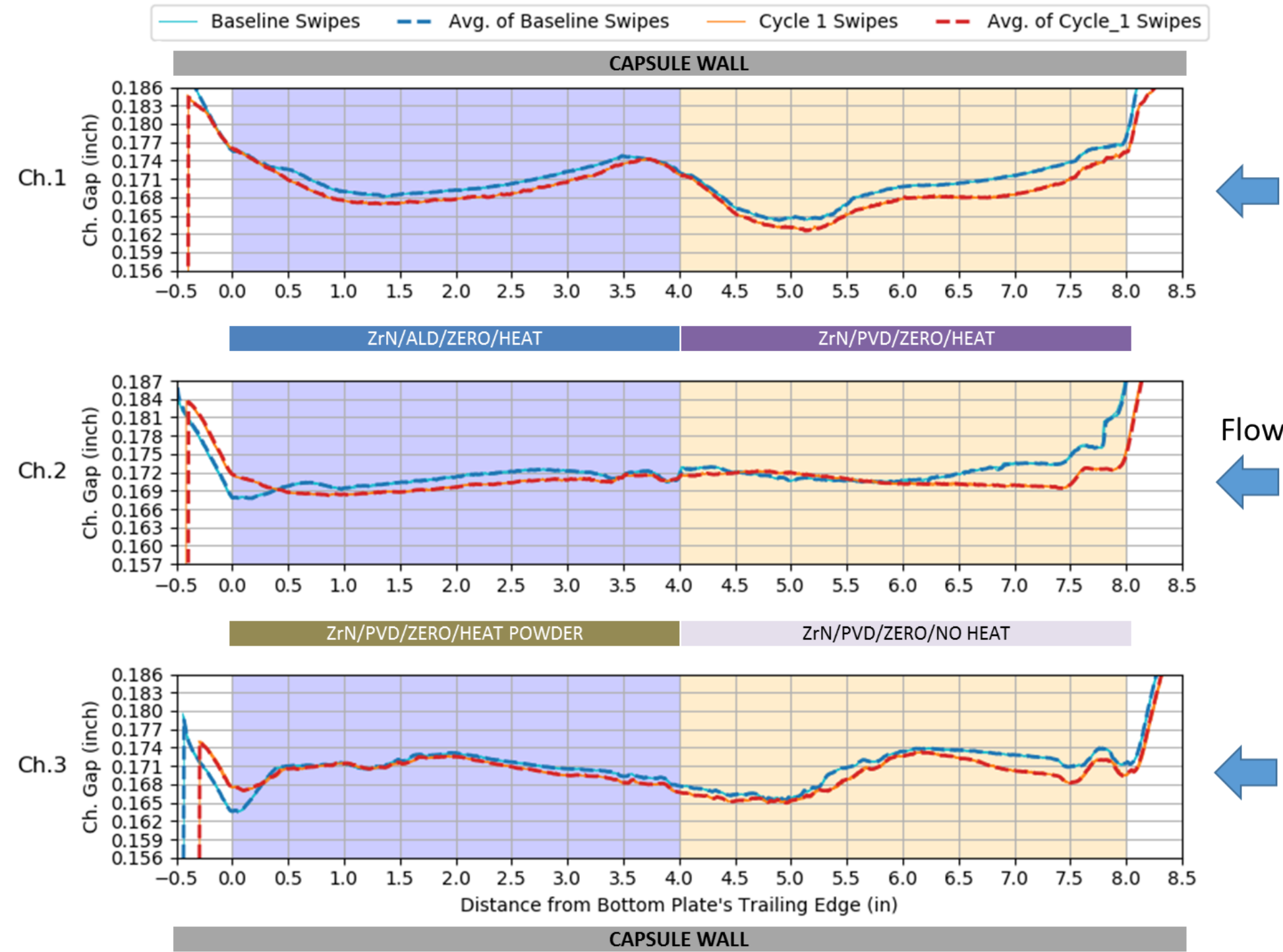

Figure 39. Baseline (blue traces) and Cycle 1 (red traces) CGP measurements for capsule EMP-4 with descriptions of the plate types between the plots of channel gap width. 

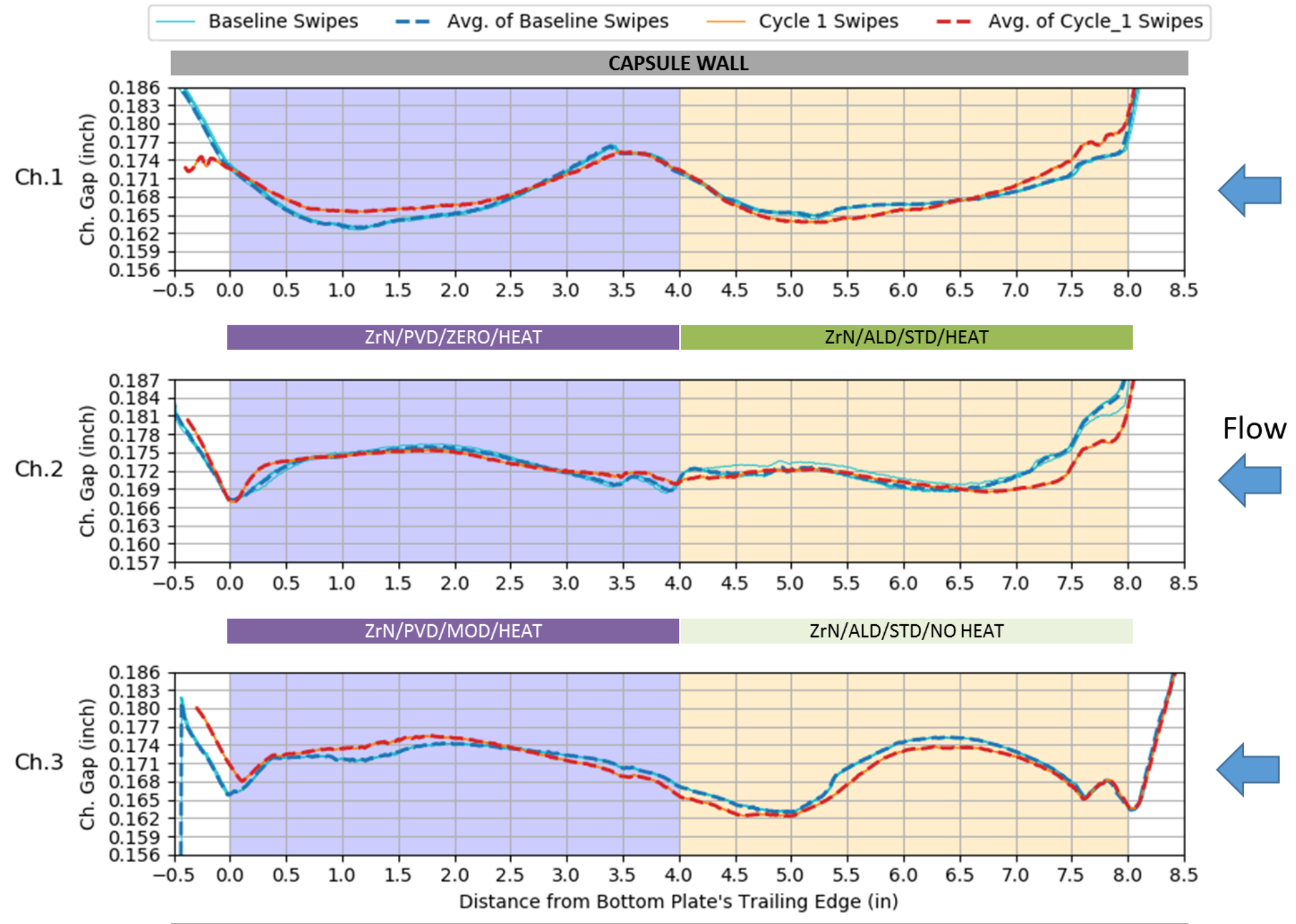

CAPSULE WALL

Figure 40. Baseline (blue traces) and Cycle 1 (red traces) CGP measurements for capsule EMP-5 with descriptions of the plate types between the plots of channel gap width. 

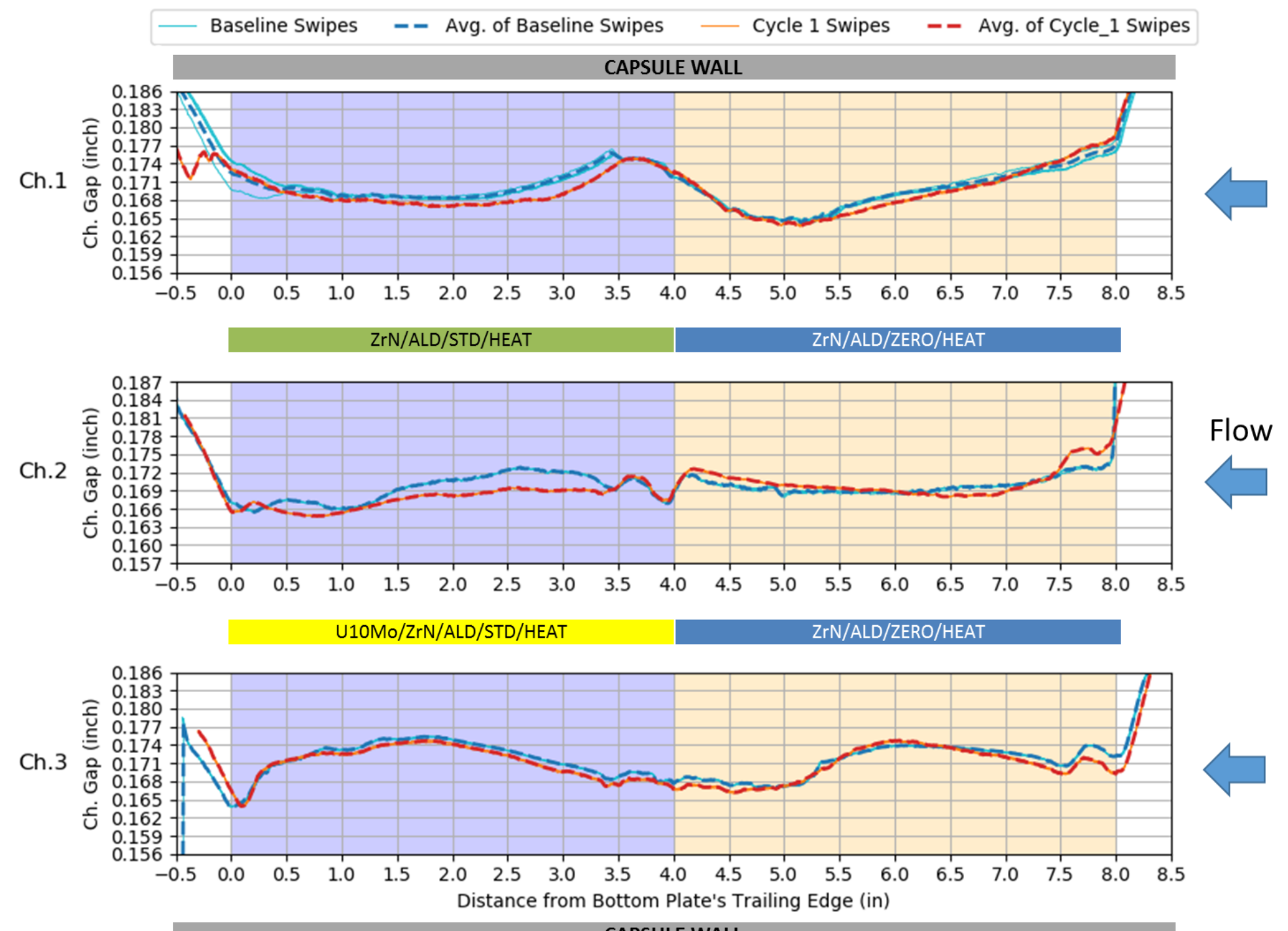
CAPSULE WALL

Figure 41. Baseline (blue traces) and Cycle 1 (red traces) CGP measurements for capsule EMP-6 with descriptions of the plate types between the plots of channel gap width. 

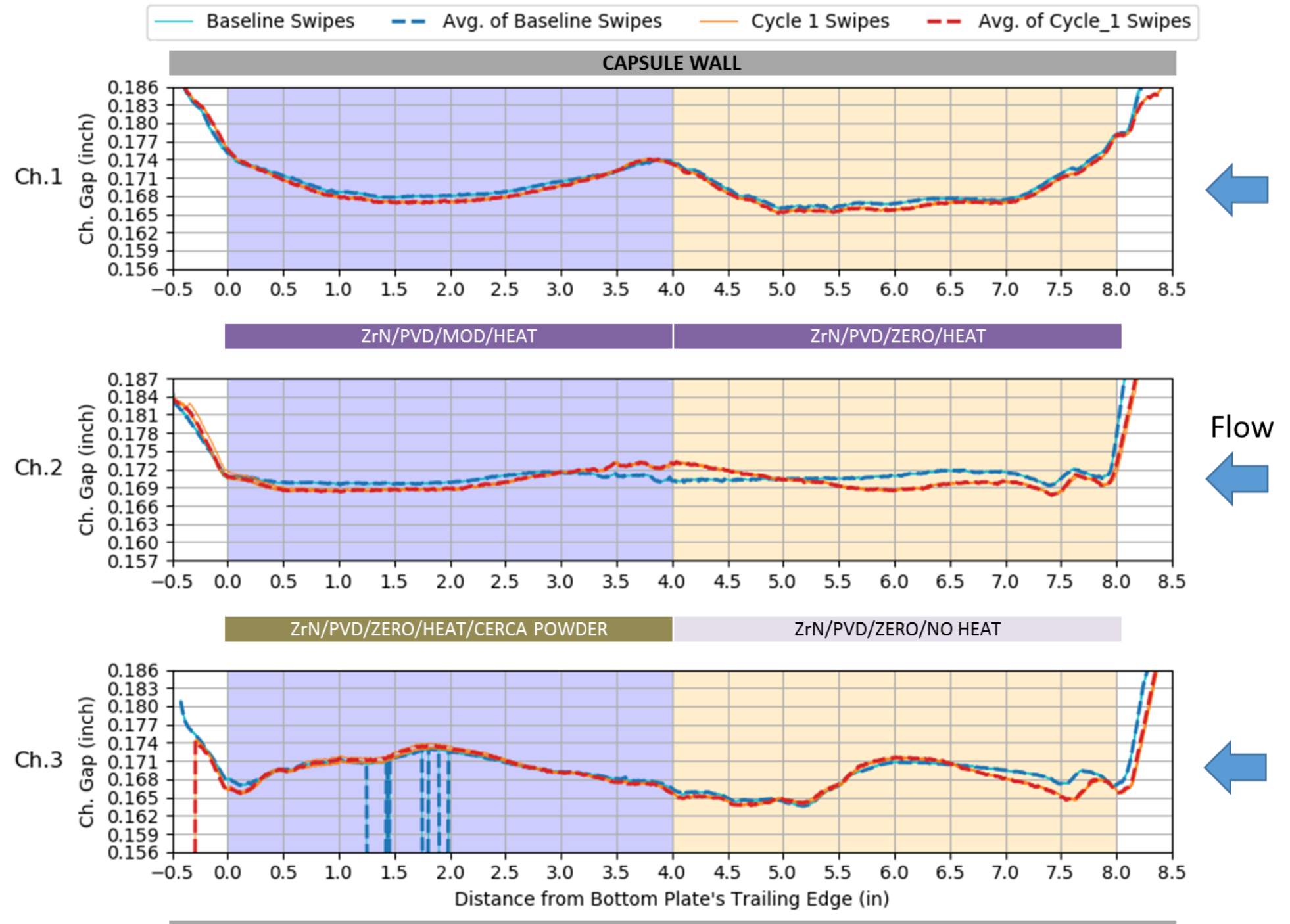
CAPSULE WALL

Figure 42. Baseline (blue traces) and Cycle 1 (red traces) CGP measurements for capsule EMP-7 with descriptions of the plate types between the plots of channel gap width. 

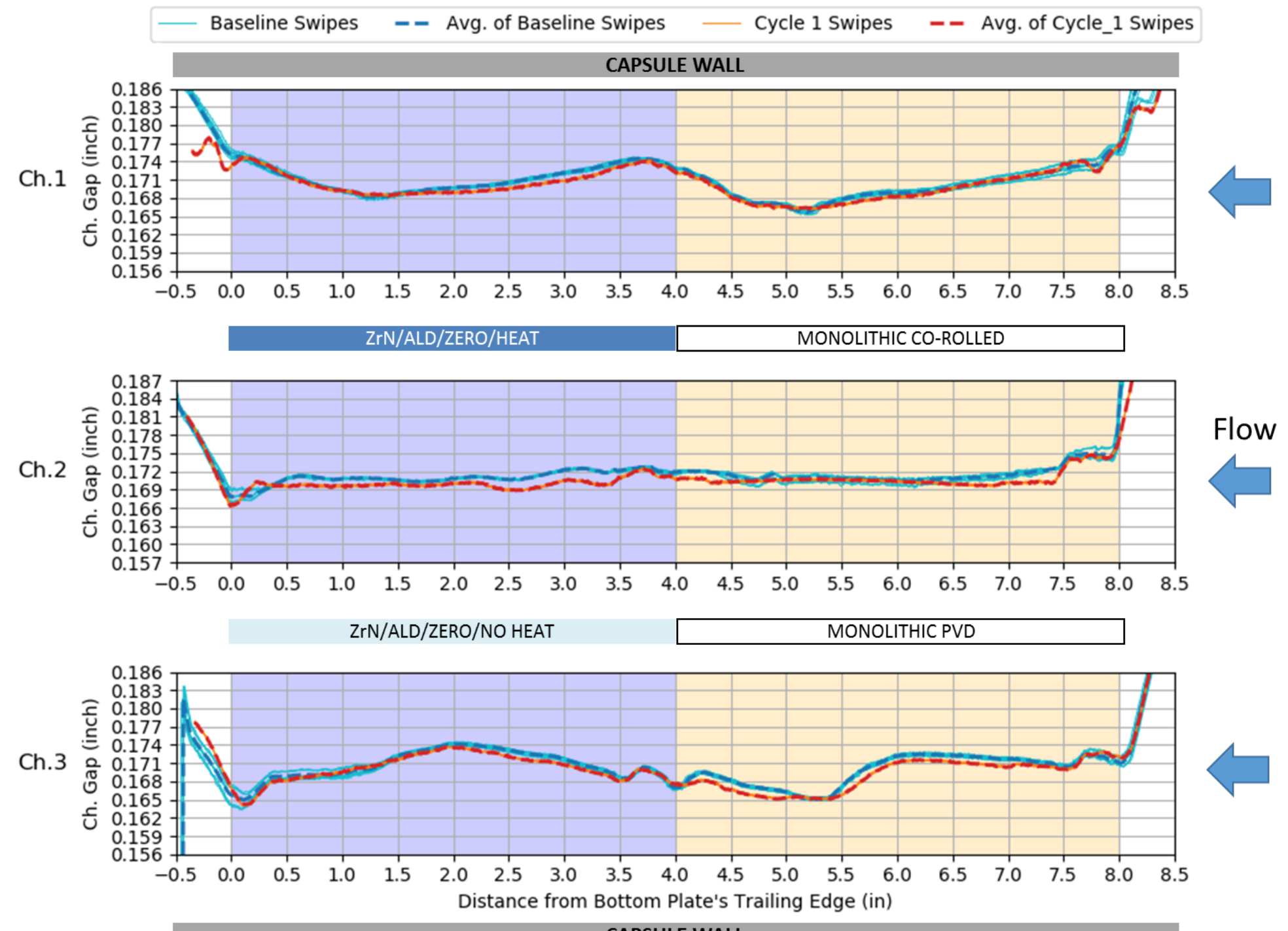

CAPSULE WALL

Figure 43. Baseline (blue traces) and Cycle 1 (red traces) CGP measurements for capsule EMP-8 with descriptions of the plate types between the plots of channel gap width. 

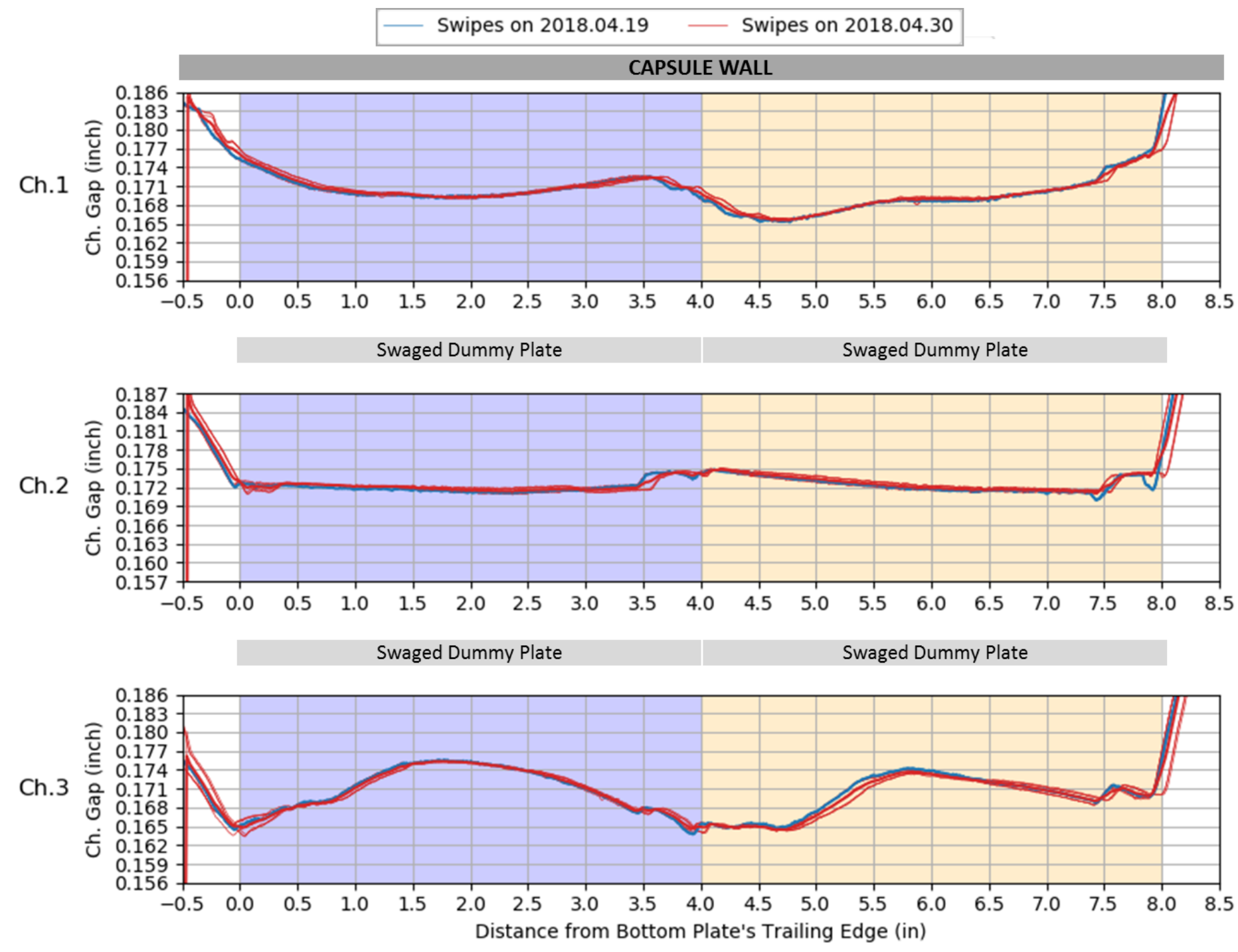

CAPSULE WALL

Figure 44. CGP measurements for the ATR Dummy capsule that contains four swaged dummy plates. 


\subsection{Change in Channel Gap Width}

Figure 45 through Figure 52 show how the channel gap changed in capsules EMP-1 through 8 after being irradiated in ATR during 162B. The change in the channel gap was calculated by subtracting the Baseline data from the Cycle 1 data (red traces). Therefore negative values in Figure 45 through Figure 52 signify that the channel gap narrowed after irradiation and positive values signify that the channel gap expanded. It is expected that the channel gaps should narrow during irradiation. Figure 45 through Figure 52 also contain data from PLATE 2.0 models [12] (blue points) that were built as part of the thermal hydraulic programmatic analysis for EMPIrE and Figure 52 also contains data from Abaqus models (green traces) of the monolithic plates that were in EMP-8 [13].

In general the CGP data shows that the channel gaps decreased, but in some cases the data indicates that the gaps expanded. Examples of the channels gaps increasing can be seen in channel 3 of EMP-1 (Figure 45), and in channels 1 and 3 of EMP-5 (Figure 49). With the exception of the "bump" in channel 3 of EMP-1, the change in channel gap is generally within the predictions of the PLATE 2.0 models within the valid data collection regions ( 0.5 to 3.5 inches and 4.5 to 7.5 inches) in channels 1 and 3 of EMP-1. The CGP data does not follow the model data as well through channel 2 of EMP-1. Similar conclusions can be drawn for the other 7 capsules.

As mentioned in the previous section, CGP measurements were taken on a dummy capsule during Cycle 1 measurements at the ATR canal. Figure 53 shows the difference between the first set of measurements taken on the dummy capsule on April 19, 2018 and the last set taken on April 30, 2018. The difference through each channel is essentially zero with very small differences near the edges of the plates, which is expected.

The change in gap distance has high fidelity and is sensitive to plate movement between measurements. The plates within the capsule did move between measurements. Thus the plates could be slightly missregistered between measurements. The miss-registration would cause sharp surface features on the plate such as small dents, pits or scratches not to exactly cancel by subtracting the bassline data from the irradiated data. The small dimples in Figure 47 for channel 1 at 0.4 and 1" and for channel 3 at 3.5" are classic examples of this effect. Another example is displayed in Figure 50 for channel 1 near 1 and 5". The dummy capsule measurements supports this miss-registration theory as shown in Figure 53 for channel 2 near 1.5". 


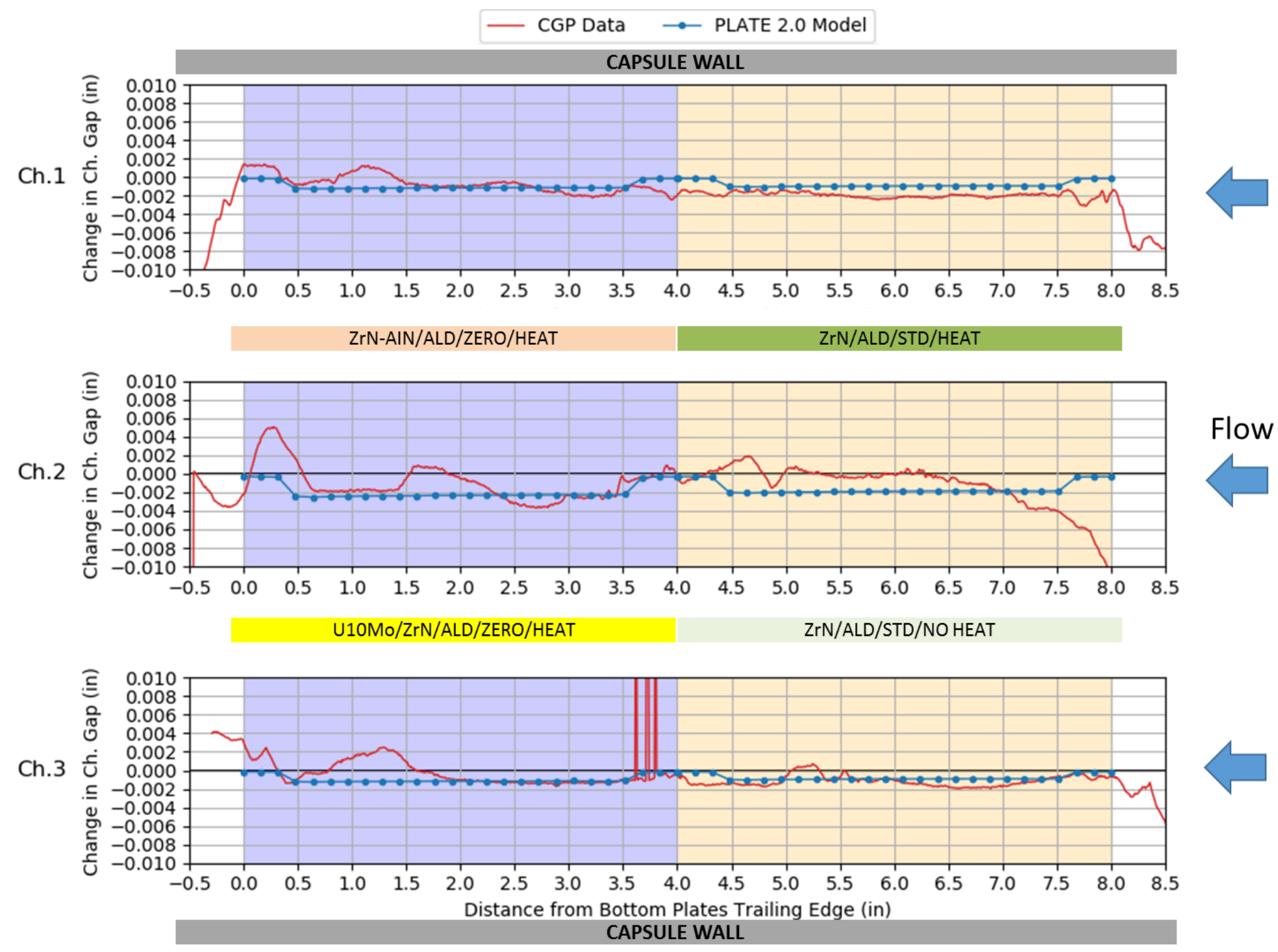

Figure 45. Red trace is change in channel gap (Cycle 1 data - Baseline data) and blue points represent modeling data from the code PLATE for EMP-1. 

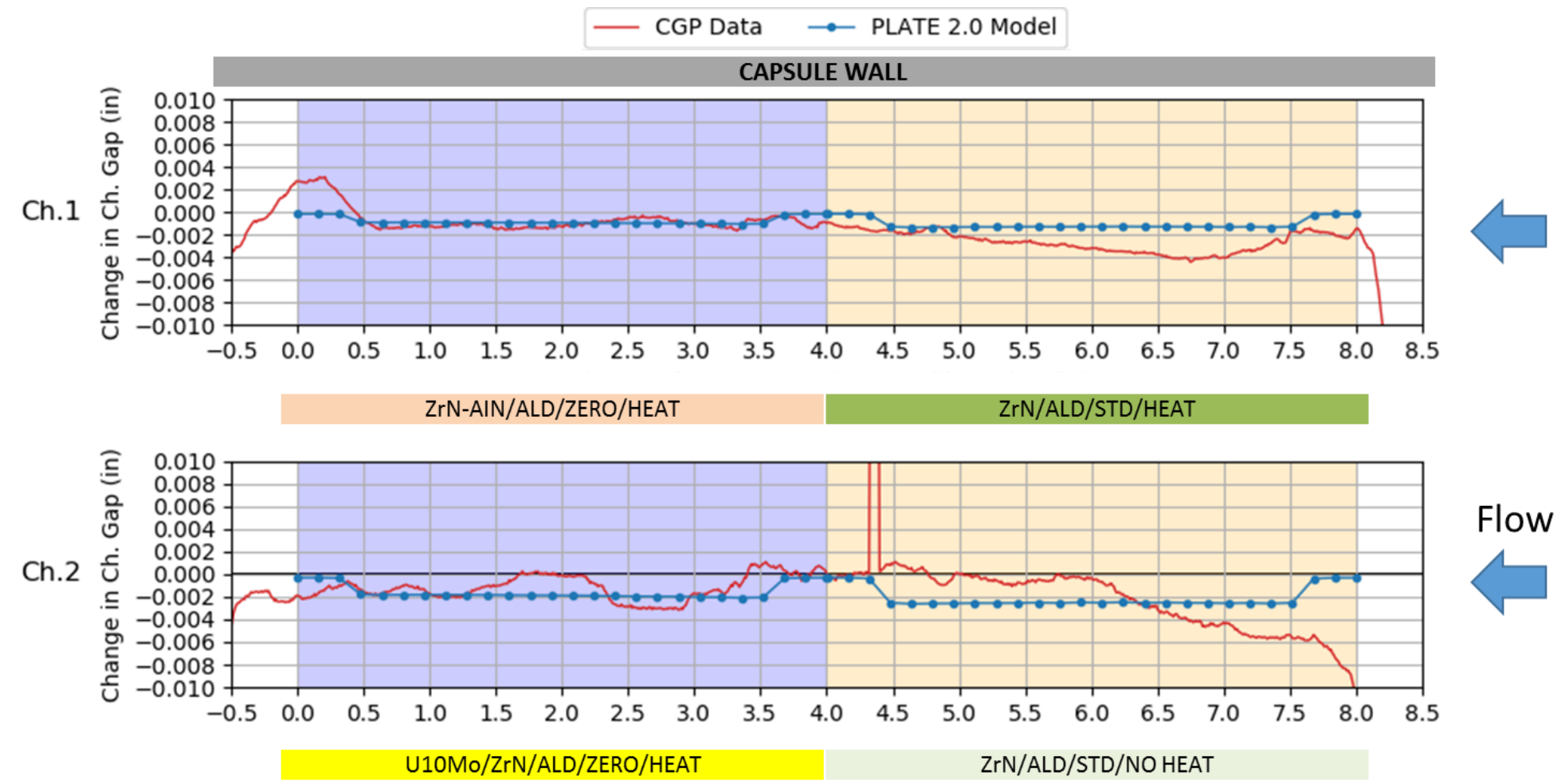

0.004

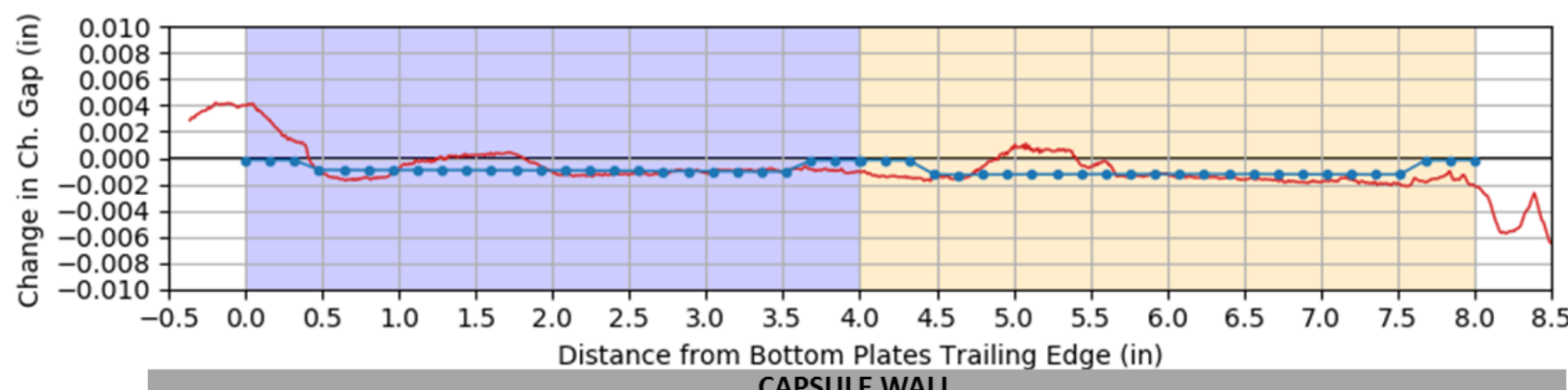

CAPSULE WALL

Figure 46. Red trace is change in channel gap (Cycle 1 data - Baseline data) and blue points represent modeling data from the code PLATE for EMP-2. 


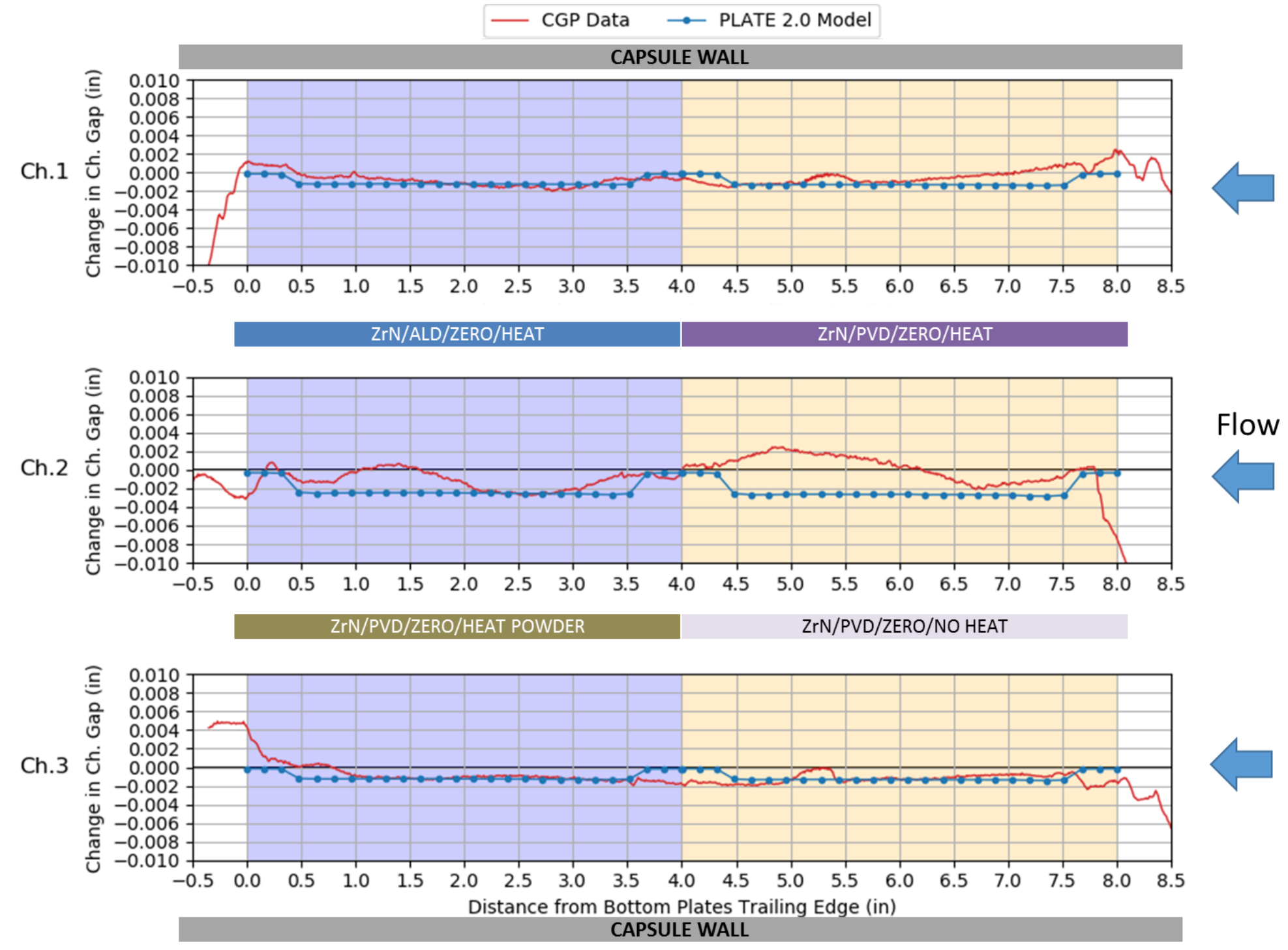

Figure 47. Red trace is change in channel gap (Cycle 1 data - Baseline data) and blue points represent modeling data from the code PLATE for EMP-3. 

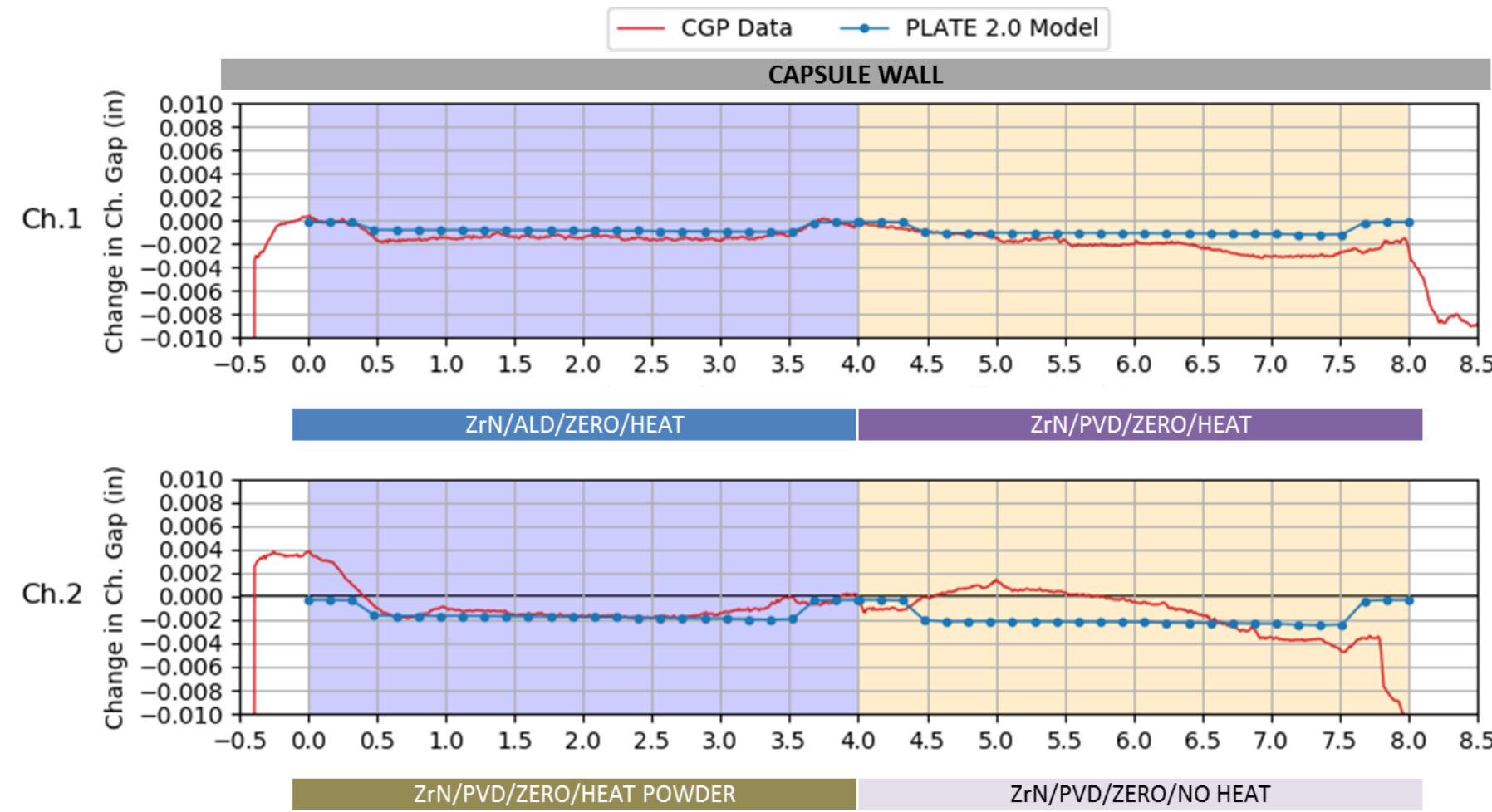

Flow

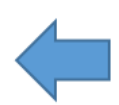

${ }^{c}{ }^{2}$

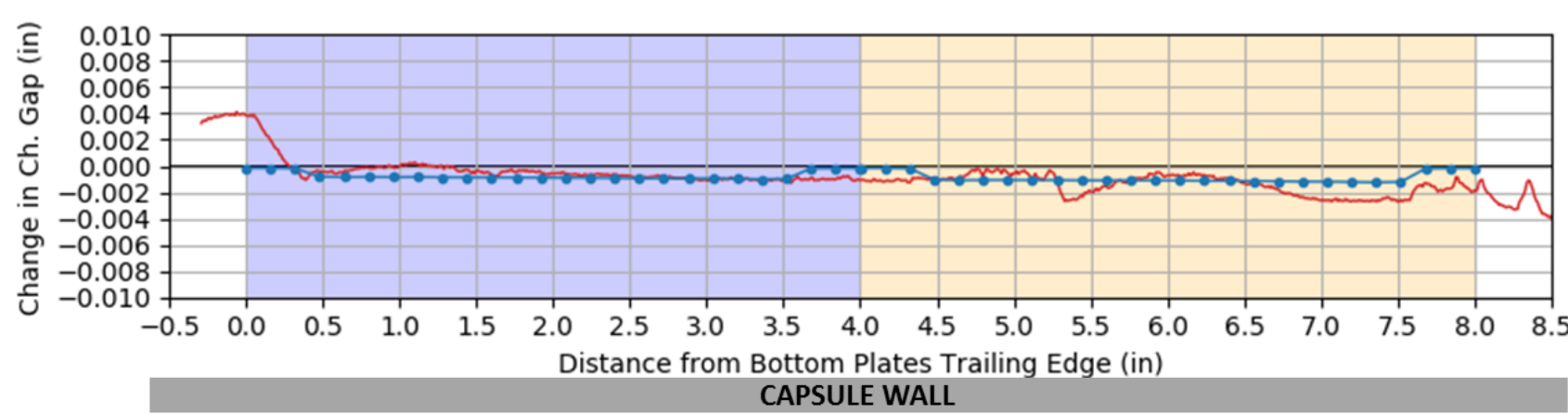

Figure 48. Red trace is change in channel gap (Cycle 1 data - Baseline data) and blue points represent modeling data from the code PLATE for EMP-4. 

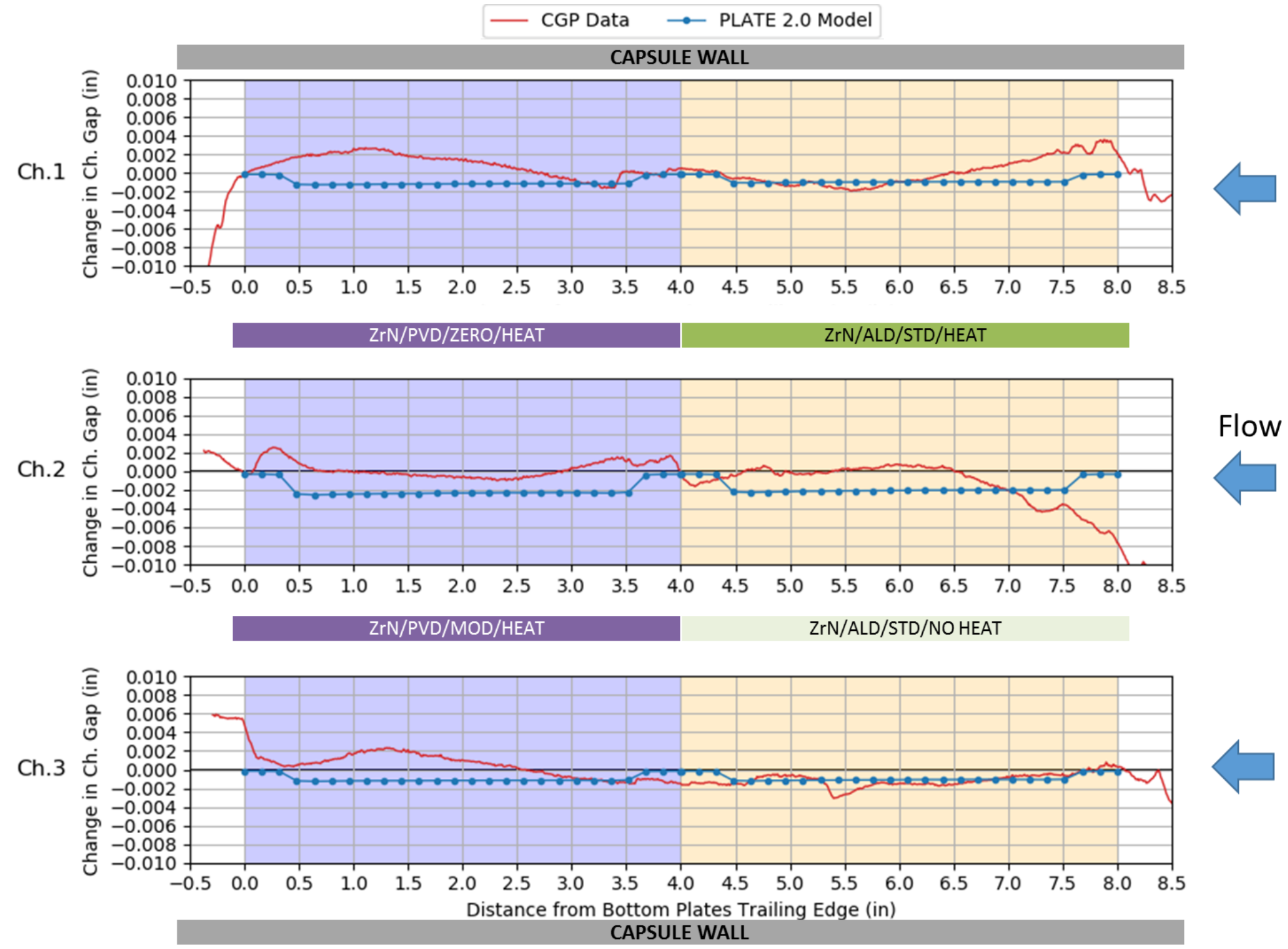

Figure 49. Red trace is change in channel gap (Cycle 1 data - Baseline data) and blue points represent modeling data from the code PLATE for EMP-5. 

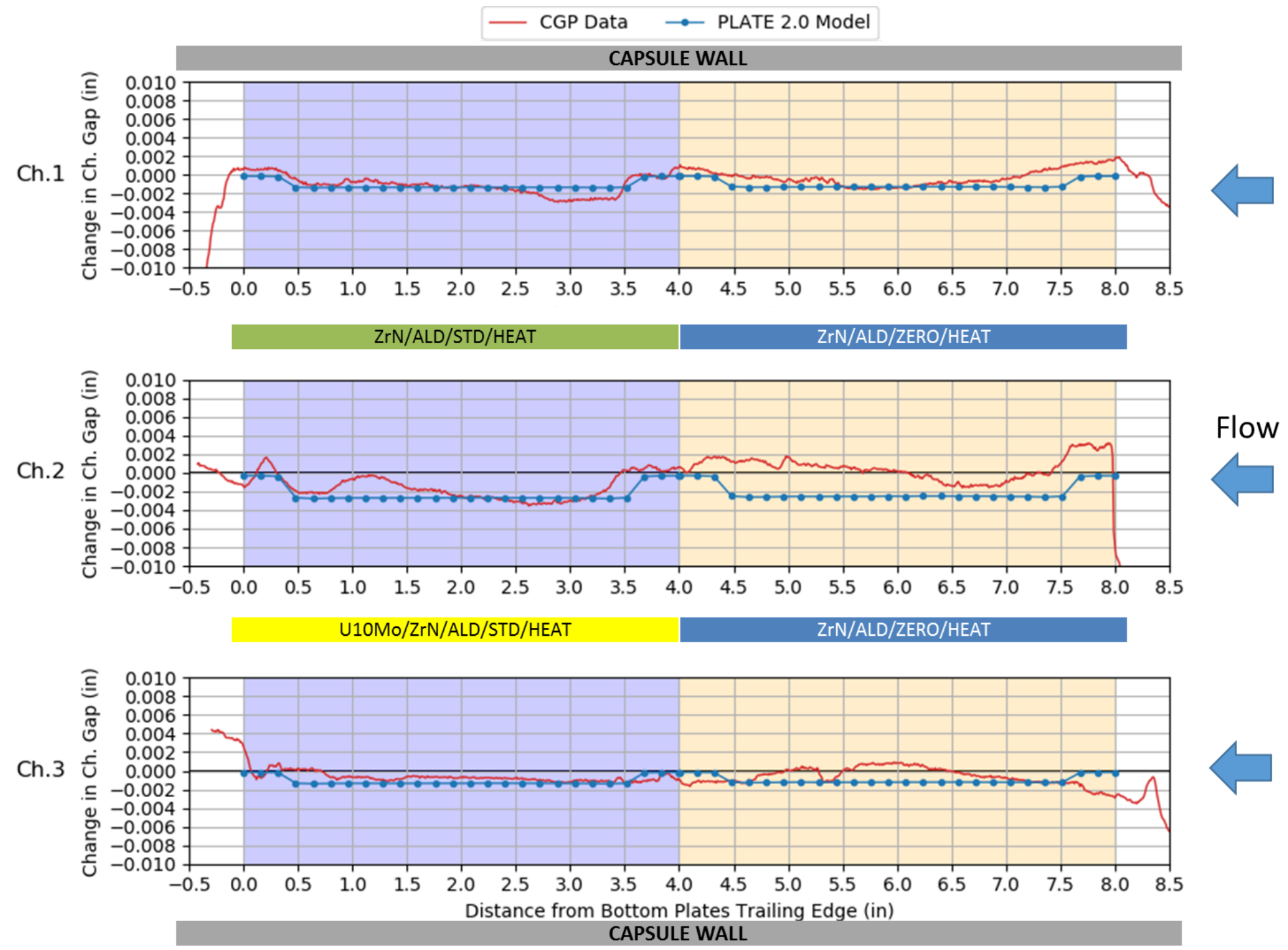

Figure 50. Red trace is change in channel gap (Cycle 1 data - Baseline data) and blue points represent modeling data from the code PLATE for EMP-6. 

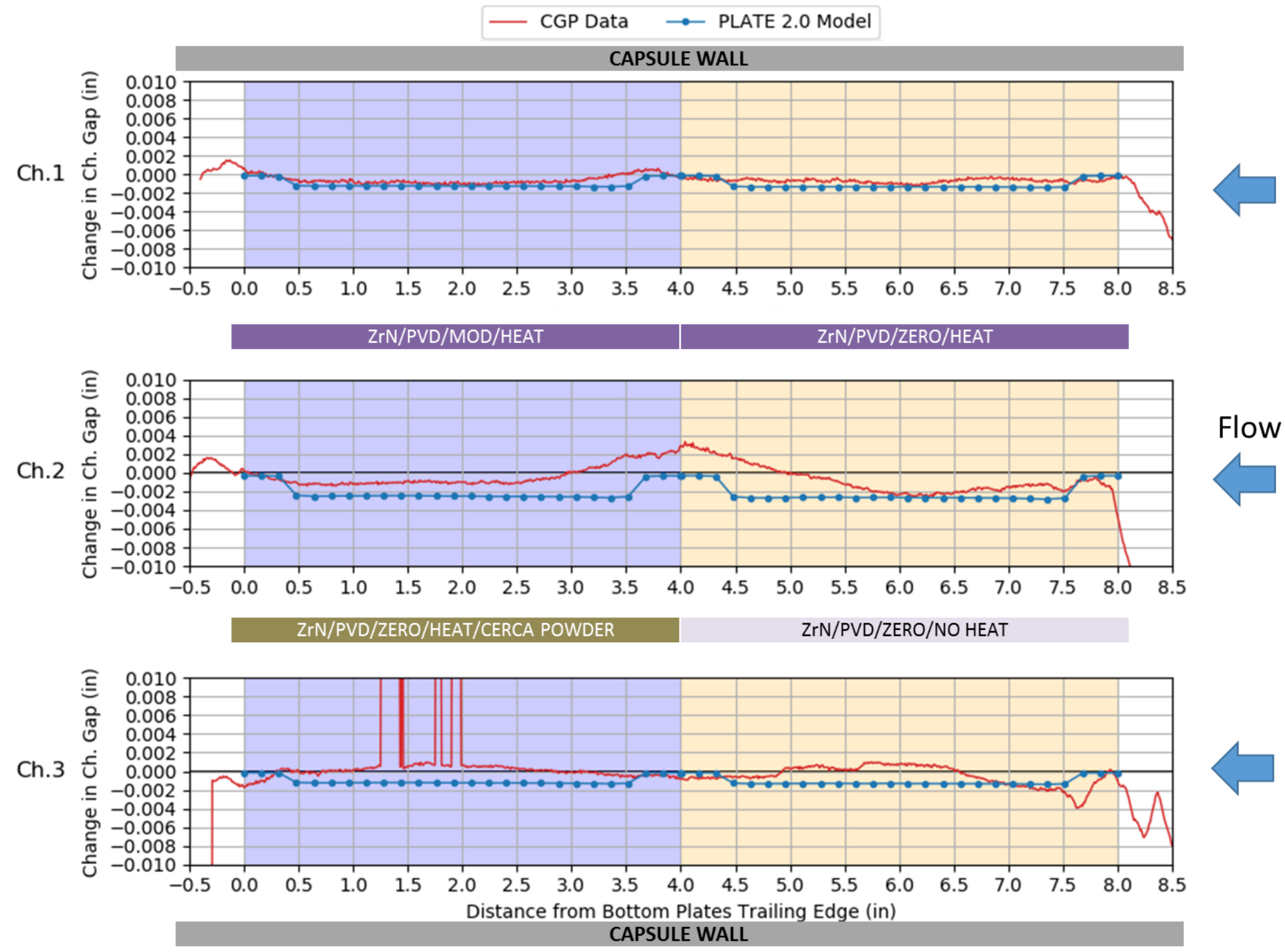

Figure 51. Red trace is change in channel gap (Cycle 1 data - Baseline data) and blue points represent modeling data from the code PLATE for EMP-7. 


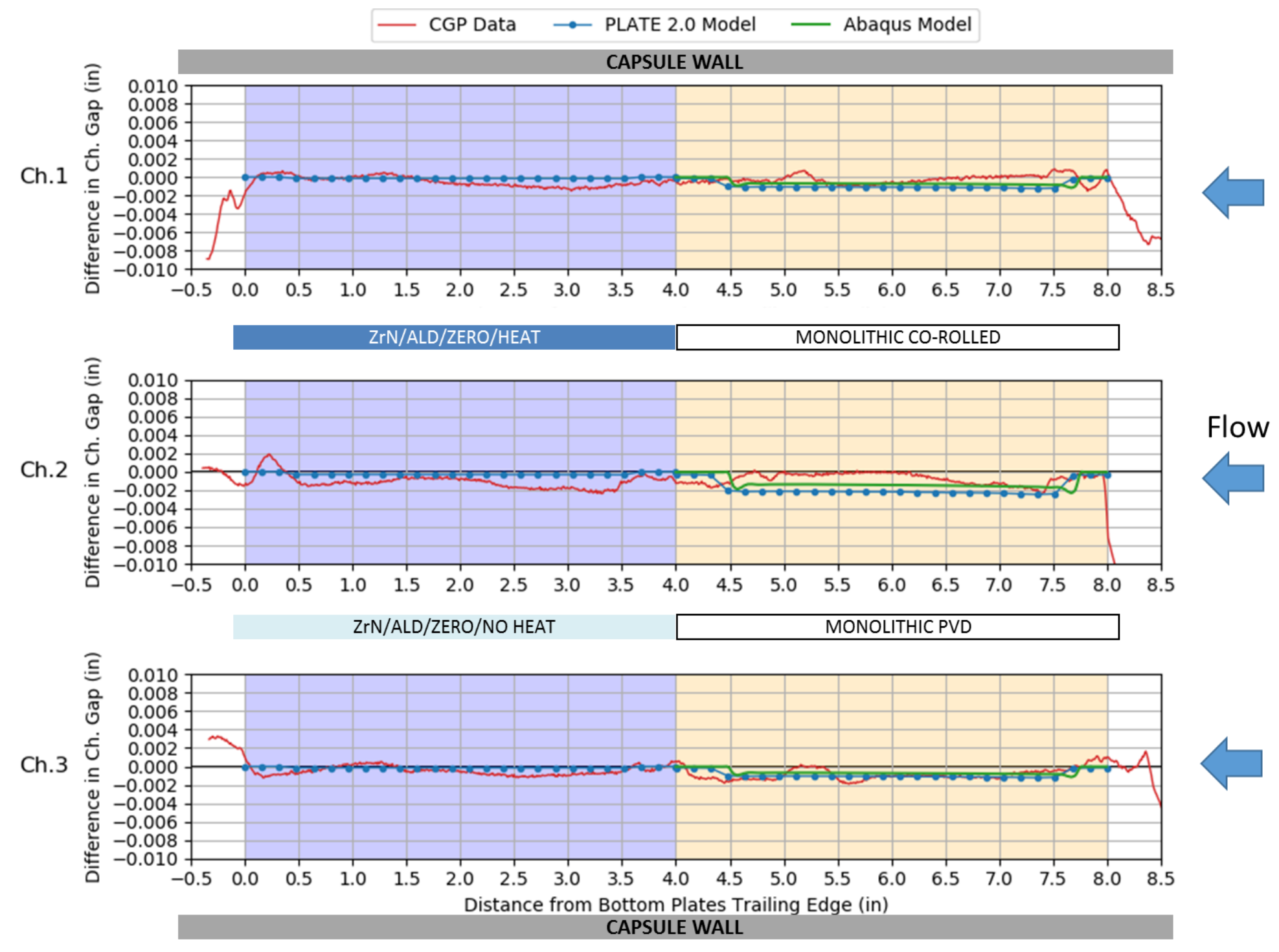

Figure 52. Red trace is change in channel gap (Cycle 1 data - Baseline data), blue points represent modeling data from the code PLATE, and green traces through the monolithic plates are from Abaqus modeling for EMP-8. 

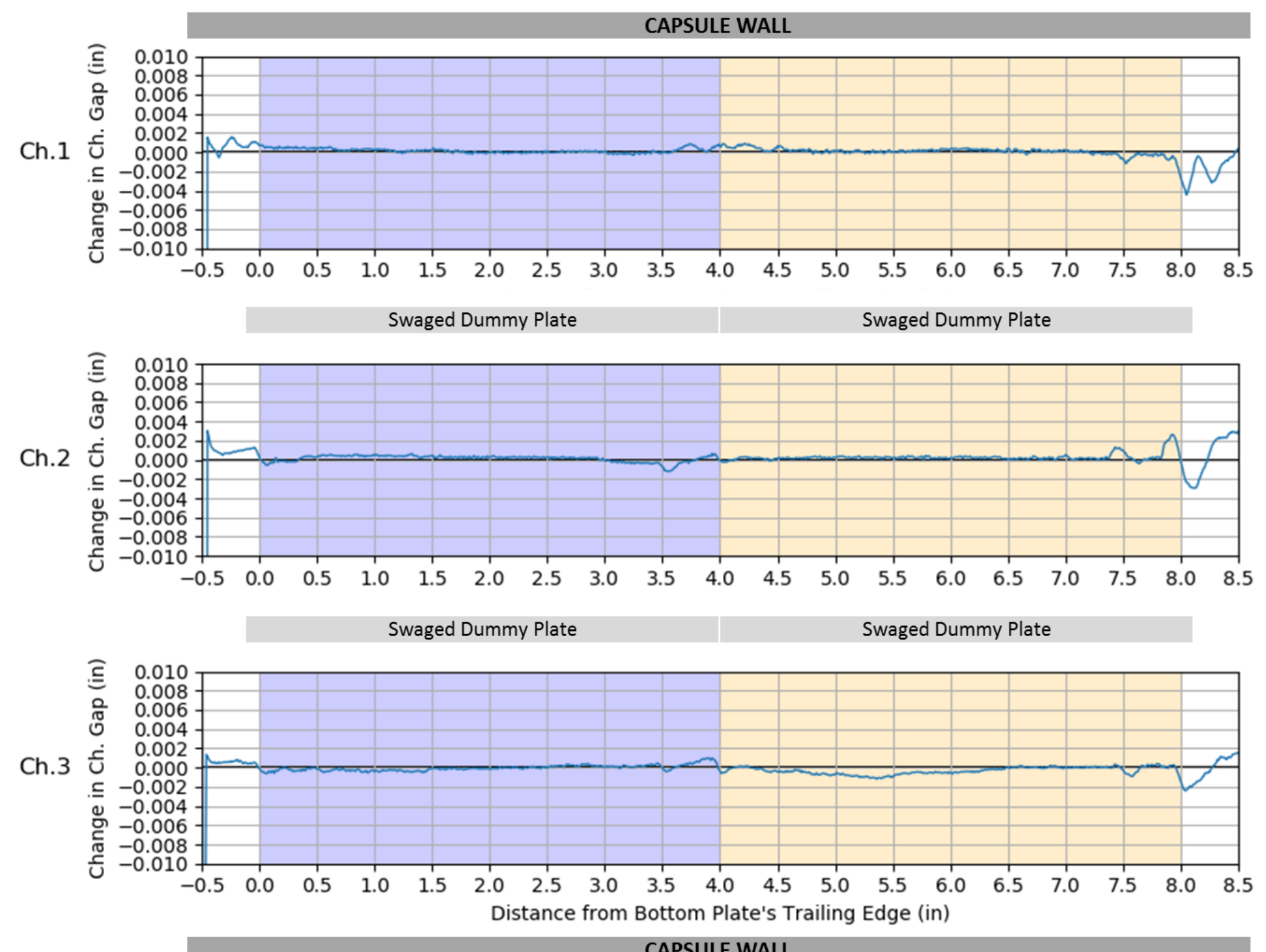

Figure 53. Change in channel gap of the ATR Dummy capsule runs on April 19, 2018 and April 30, 2018. 


\section{CONCLUSIONS}

The physical basis of the channel gap width measurement has been discussed. The measurement technique is based on ultrasonic time of flight between two transducers and the CGP calibration process has been presented. The measurement technique has been shown to be sensitive to a number of parameters, temperature, geometry changes in the measurement wand and ultrasonic reverberations. Techniques to mitigate the confounding influences have been discussed. The main technique to assess data confidence is monitor the ultrasonic amplitude of the return signal. Significant changes in the resulting amplitude signatures indicate the quality and confidence of the resulting data.

The summary results from a Repeatability and Reproducibility report have been presented as well as probe performance. The expected gap width repeatability and reproducibility between ATR runs are $0.0076 \mathrm{~mm}(0.0003 ")$ and $0.023 \mathrm{~mm}(0.0009 ")$ respectively with a three run gap width STD of $0.021 \mathrm{~mm}$ $(0.00083$ "). These statistical values along with the amplitude signatures provide a reliable indication of the data quality.

The channel gap probe data collected on the eight EMPIrE capsules irradiated during ATR cycle 162B has been shown and briefly discussed. Baseline CGP data (obtained before the capsules were irradiated) and Cycle 1 CGP data (data collected after irradiation in ATR) were compared and the change in the channel gap was calculated (Cycle 1 - Baseline) to more directly show how the channel gaps changed due to irradiation. A limiting factor to the accuracy and repeatability of the gap measurements is the missregistration of the plates between measurements. Small surface surface features can not be fully compensated by the subtracting the baseline measurements and show up as small dimples in the gap measurement data. The CGP data was then compared to models built in PLATE 2.0 (all capsules) and Abaqus (monolithic plates in EMP-8). For the most part, the CGP data followed the model's predictions, however some of the CGP data showed the channel gaps increasing, which was not expected and thus was not predicted by the models. In terms of certifying the capsules for reinsertion, the capsule were uninteresting (no indications of concern were found). 


\section{REFERENCES}

1. Daniele Marioli, Claudio Narduzzi, Carlo Offelli, Dario Petri, Emilio Sardini, and Andrea Taroni, "Digital Time-of-Flight Measurement for Ultrasonic Sensors," IEEE Transactions On Instrumentation And Measurement, Vol. 41, No. 1, pp. 93 - 97, February 1992. https://ieeexplore.ieee.org/stamp/stamp.jsp?arnumber=126639

2. Wang XF1, Tang ZA., "A novel method for digital ultrasonic time-of-flight measurement," Rev Sci Instrum. 2010 Oct;81(10):105112. doi: 10.1063/1.3493046. https://aip.scitation.org/doi/pdf/10.1063/1.3493046

3. GDE-816, "UT Operator's Guide For Channel Gap Probe"

4. PLN-5214, "Qualification Plan For The Channel Gap Measurement System"

5. LI-806, "Channel Gap Probe Development and Operation"

6. https://en.wikipedia.org/wiki/Newton\%27s_law_of_cooling

7. http://resource.npl.co.uk/acoustics/techguides/soundpurewater/bilaniuk.html, 36 point interpolation.

8. Smith, James A., "Repeatability and Reproducibility Estimation of Mini Plate Channel Gap Probe TEV-3363", 12/19/2017, Rev. 05

9. ATSM International, Standard Practice for Conduction an Interlaboratory Study to Determine the Precision of a Test Method, E691-16.

10. Empire 8 data storage location, Y:IRERTR Working Files\Channel Gap Probe Files $D$ Data AquisitionไEmpirelFreshFuellEMP-8

11. "PLN-5023 European Mini-Plate Irradiation Experiment (EMPIRE) Execution Plan," Idaho National Laboratory, 2018.

12. Crawford, Doug, "ECAR-3373 Thermal Hydraulic Programmatic Analysis of the EMPIRE Experiment in the ATR," 04/06/2018, Rev. 1

13. Ozaltun, Hakan, "INL/INT-18-45037 Thickness Estimates for Monolithic Plates of the Empire Experiments", 04/30/2018, Rev. 0 


\section{Appendix A - EMPIrE Capsules 9 through 12}
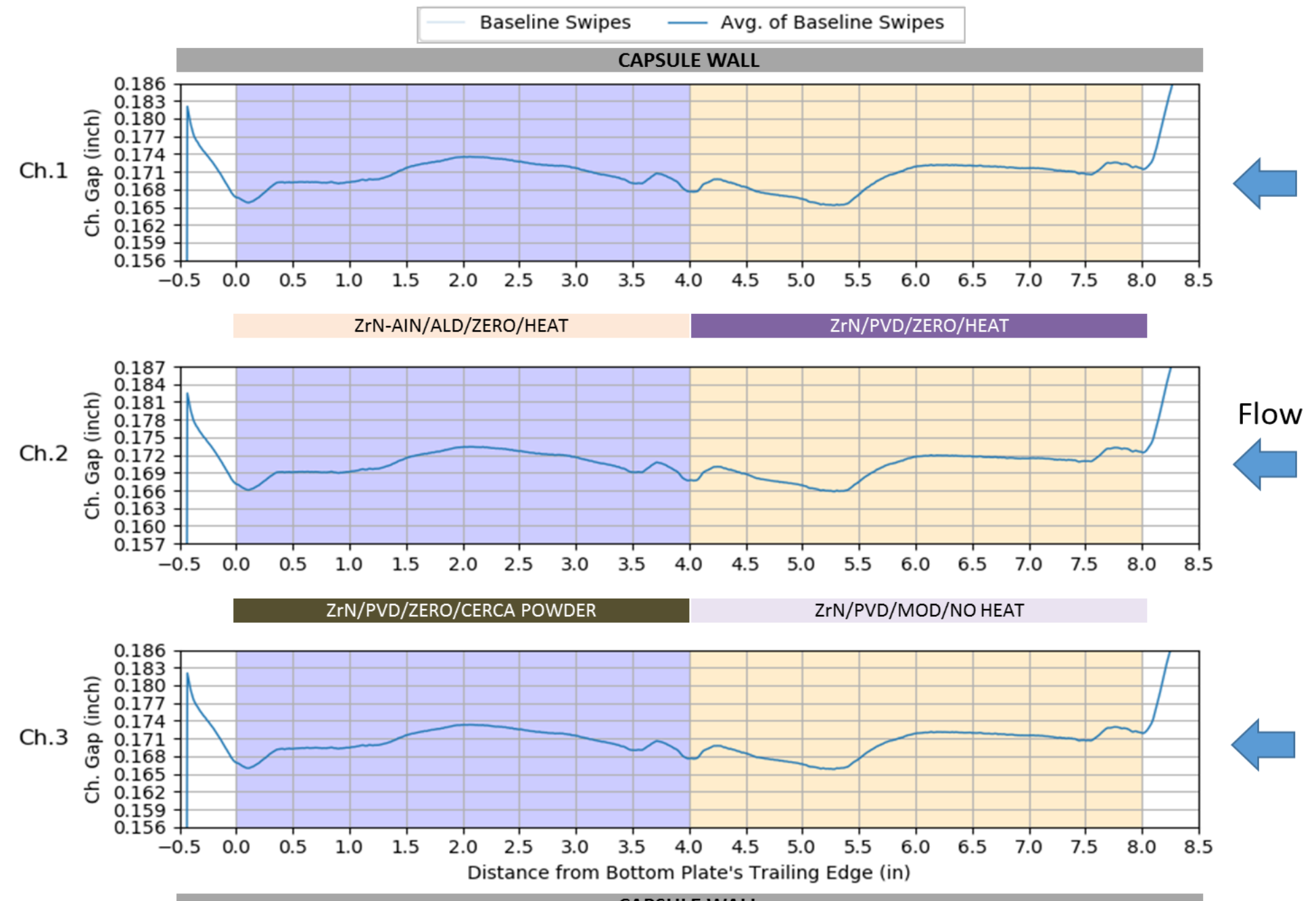

Figure A1. Baseline (blue traces) CGP measurements for capsule EMP-9 with descriptions of the plate types between the plots of channel gap width. 

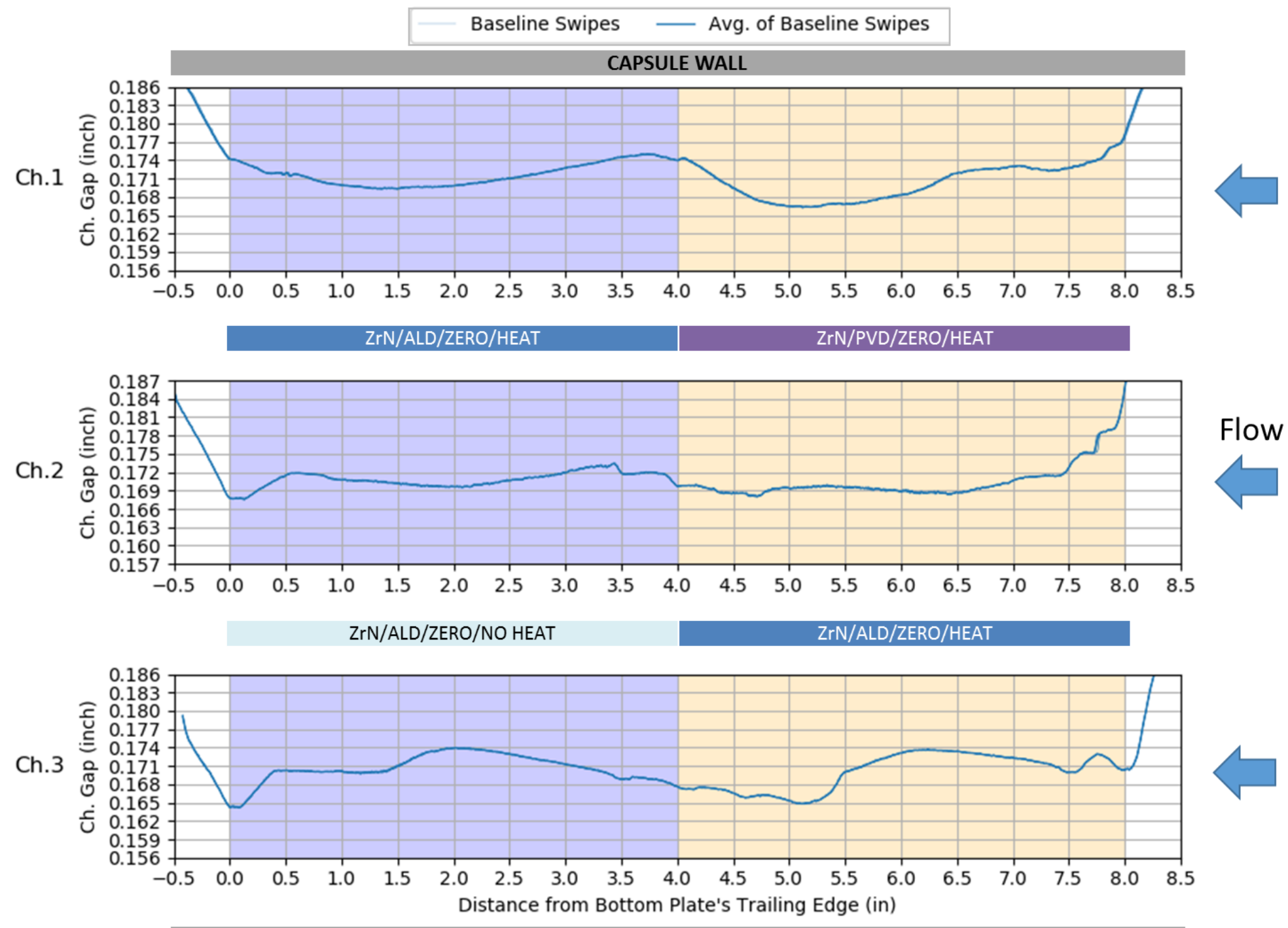
CAPSULE WALL

Figure A2. Baseline (blue traces) CGP measurements for capsule EMP-10 with descriptions of the plate types between the plots of channel gap width. 

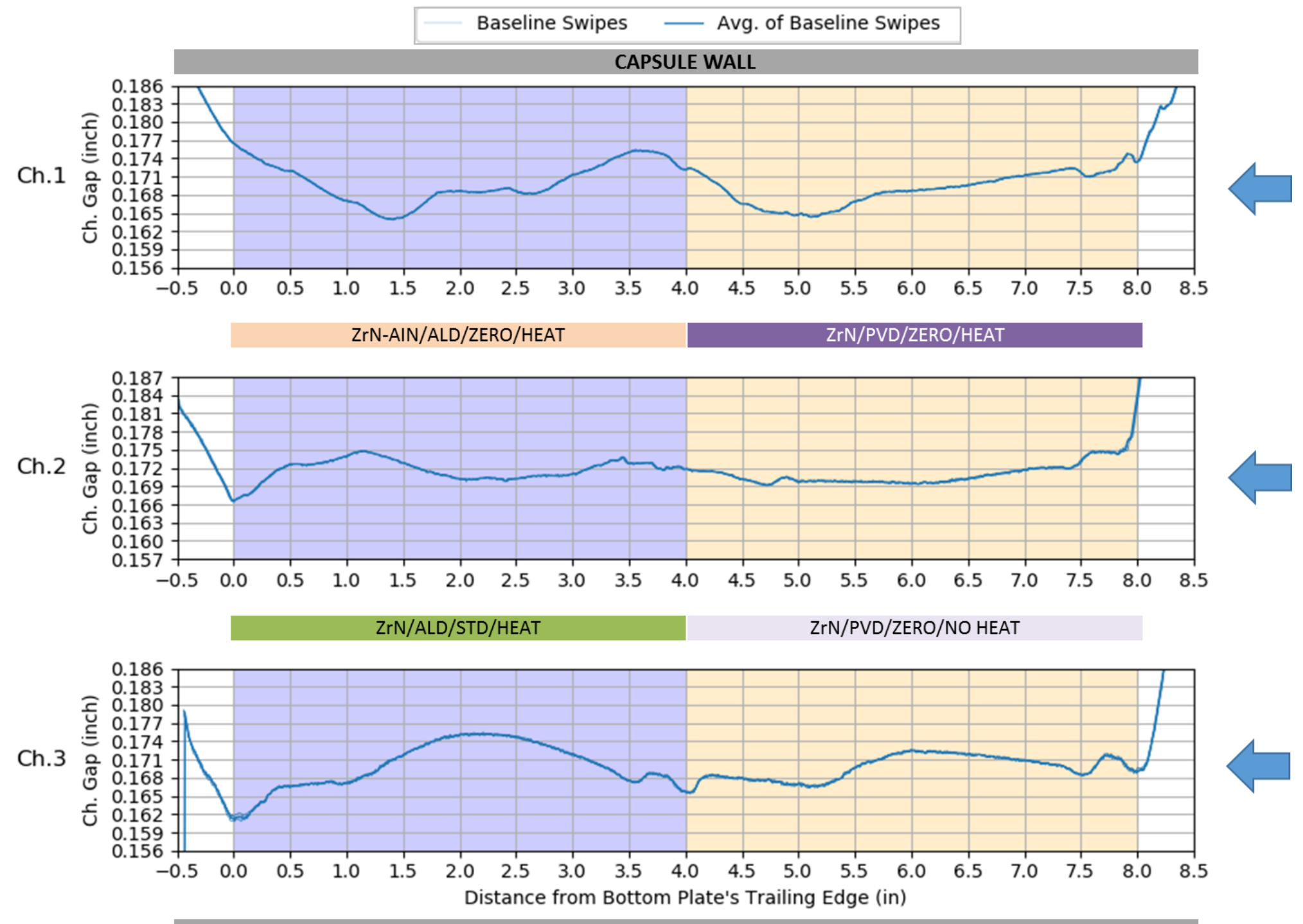

Figure A3. Baseline (blue traces) CGP measurements for capsule EMP-11 with descriptions of the plate types between the plots of channel gap width. 

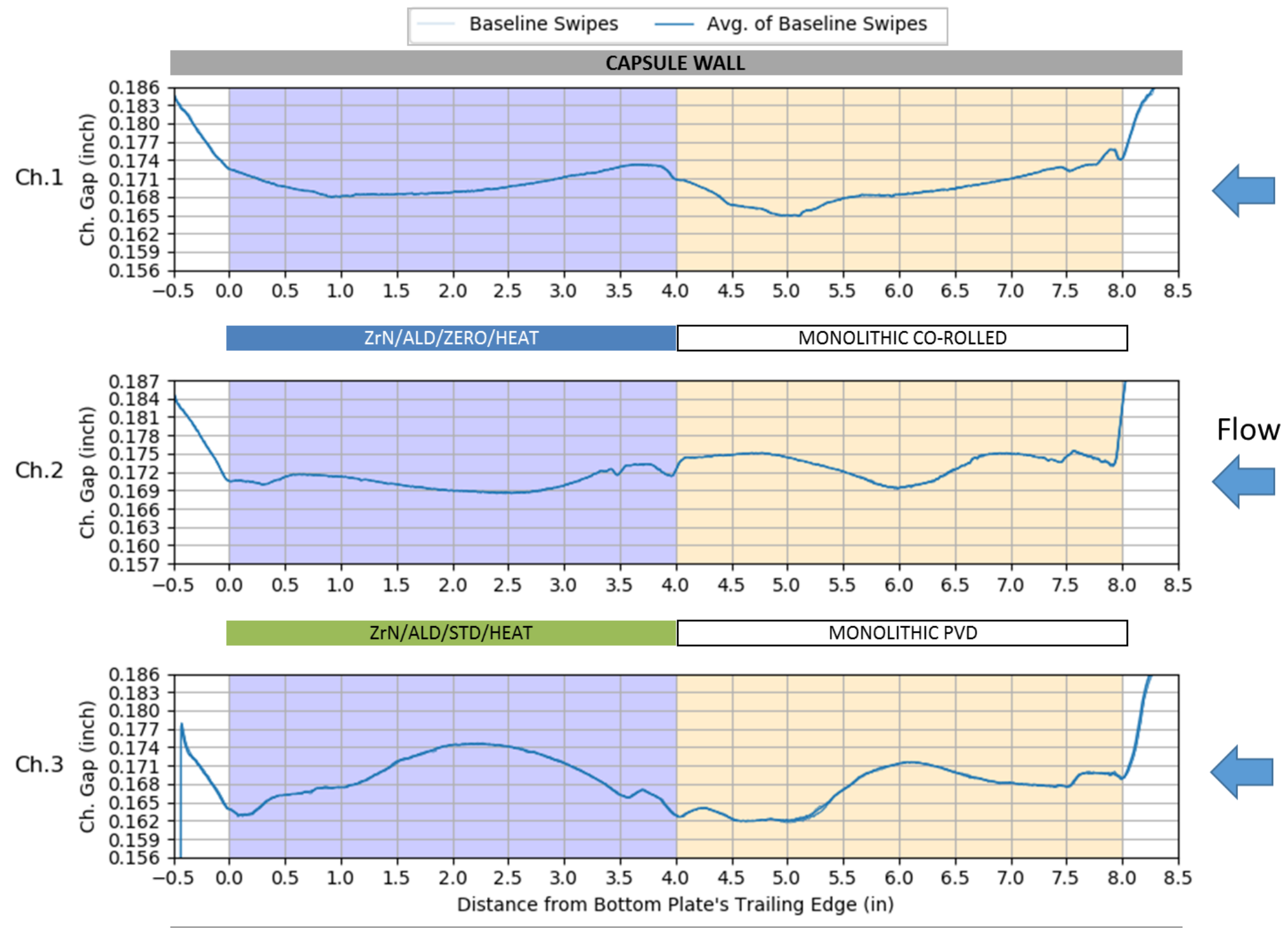

Figure A4. Baseline (blue traces) CGP measurements for capsule EMP-12 with descriptions of the plate types between the plots of channel gap width. 